\title{
THE MYCOBIOTA OF SAMANLI MOUNTAINS IN TURKEY
}

\author{
Hasan Hüseyin DOĞAN ${ }^{1 *}$, Öyküm ÖZTÜRK ${ }^{2}$, Murad Aydın ŞANDA ${ }^{3}$ \\ ${ }^{1}$ Selçuk University, Science Faculty, Biology Department, Konya, TURKEY \\ ${ }^{2}$ Hacettepe University, Science Faculty, Biology Department, Ankara, TURKEY \\ ${ }^{3}$ Muş Alparslan University, Science and Letter Faculty, Molecular Biology and Genetic Department, Muş, TURKEY
}

Cite this article as:

DOĞAN, H.H., ÖZTÜRK, Ö \& ŞANDA, M.A. 2021. The mycobiota of Samanlı Mountains in Turkey. Trakya Univ J Nat Sci, 22(2): 215-243, DOI: $10.23902 /$ trkjnat.947894

Received: 04 June 2021, Accepted: 31 August 2021, Online First: 04 October 2021, Published: 15 October 2021

Edited by:

Neeven Geweely

\section{*Corresponding Author:}

Hasan Hüseyin Doğan

hhuseyindogan@yahoo.com

\section{ORCID iDs of the authors:}

HHD. orcid.org/0000-0001-8859-0188

ÖÖ. orcid.org/0000-0001- 9846-3668

MAŞ. orcid.org/0000-0001-8843-4361

Key words:

Fungal distribution

Samanlı Mountains

New records

Turkey

\begin{abstract}
The Mycobiota of Samanl1 Mountains were investigated in this study. Specimens were collected during 3 years between November 2012 and November 2015. 510 macrofungal taxa belonging to 197 genera within 84 families were recorded with field and laboratory studies. Of these, 37 genera and 57 taxa belong to Ascomycota, while 160 genera and 453 taxa belong to Basidiomycota. Nine species were found for the first time in Turkey from Basidiomycota. These taxa are Amanita subnudipes (Romagn.) Tulloss, Hebeloma quercetorum Quadr., Hygrocybe obrussea (Fr.) Wunsche, Lactarius mediterraneensis Llistosella \& Bellù, Lactifluus glaucescens (Crossl.) Verbeken, Russula lilacea Quél., R. rubra (Lam.) Fr., Stereopsis reidii Losi \& A. Gennari and Tricholoma roseoacerbum A. Riva. The Stereopsidaceae family and the genus Stereopsis D.A. Reid was found for the first time in Turkey.

The richest families in terms of the number of taxa are Russulacea with 58 taxa (11.3\%), Agaricaceae with 46 taxa (8.9\%), Tricholomataceae with 43 taxa $(8.4 \%)$, Boletaceae with 32 taxa (6.2\%), Polyporaceae with 23 taxa (4.5\%) and the most crowded genera are Russula Pers. with 41 taxa (8\%), Tricholoma (Fr.) Staude with 26 taxa (5\%), Amanita Dill. ex Boehm. with 19 taxa (3.7\%), Lactarius Pers. with 16 taxa (3.1\%) and Inocybe (Fr.) Fr. with 14 taxa $(2.7 \%)$. The ecological status of the species is as follows; $245(48 \%)$ are saprobe, $226(45 \%)$ are mycorrhizal, $20(3.7 \%)$ are lignicolous, 18 (2.9\%) are parasite, and one is entomopathogenic. Habitat distribution in the research area is as follows: 300 species in Abies nordmanniana (Stev.) Spach. subsp. bornmuelleriana (Mattf.) Coode \& Cullen forest, 295 species in Fagus orientalis Lipsky forest, 125 species in Quercus spp. forest, 88 species in Pinus nigra J.F.Arnold forest, 56 species in Castanea sativa Mill. forest, 53 species in Pinus sylvestris L. forest, 49 species in Carpinus orientalis Mill. forest and 24 species in Pinus maritima Lam. forest.
\end{abstract}

Özet: Bu çalışmada Samanlı dağlarının mikobiotası araştırılmıştır. Örnekler Kasım 2012 ve Kasım 2015 arasında 3 yıl boyunca toplanmıştır. 84 familya ve 197 cinse ait 510 makromantar taksonu belirlenmiştir. Bunlardan, 37 cins ve 57 takson Ascomycota'ya aitken 160 cins ve 453 takson ise Basidiomycota'ya aittir. Basidiomycota'dan 9 tür Türkiye'de ilk kez bulunmuştur. $\mathrm{Bu}$ taksonlar Amanita subnudipes (Romagn.) Tulloss, Hebeloma quercetorum Quadr., Hygrocybe obrussea (Fr.) Wunsche, Lactarius mediterraneensis Llistosella \& Bellù, Lactifluus glaucescens (Crossl.) Verbeken, Russula lilacea Quél., R. rubra (Lam.) Fr., Stereopsis reidii Losi \& A. Gennari ve Tricholoma roseoacerbum A. Riva.'dur. Stereopsidaceae familyası ve Stereopsis D.A. Reid cinsi Türkiye'de ilk kez belirlenmiştir.

Tür sayısı bakımından en zengin familyalar Russulaceae'den 58 takson $(\% 11,3)$, Agaricaceae'den 46 takson $(\% 8,9)$, Tricholomataceae'den 43 takson $(\% 8,4)$, Boletaceae'den 32 takson $(\% 6,2)$, Polyporaceae'den 23 takson $(\% 4,5)$ dur. En zengin cinsler ise Russula Pers. 41 takson (\%8), Tricholoma (Fr.) Staude 26 takson (\%5), Amanita Dill. ex Boehm. 19 takson (\%3,7), Lactarius Pers. 16 takson (\%3,1) veInocybe (Fr.)Fr. 14 takson $(\% 2,7)$ 'dur. Türlerin ekolojik durumları şu şekildedir; 245 (\%48) saprop, 226 (\%45) mikorizal, $20(\% 3,7)$ lignikolar, $18(\% 2,9)$ parazit, ve bir tür entomopatojeniktir. Araştırma alanındaki habitat dağılımı aşağıdaki gibidir; 300 takson Abies nordmanniana (Stev.) Spach.subsp. bornmuelleriana (Mattf.) Coode \& Cullen ormanında, 295 takson Fagus orientalis Lipsky ormanında, 125 takson Quercus spp. ormanında, 88 takson Pinus nigra J.F.Arnold ormanında; 56 takson Castanea sativa Mill. ormanında; 53 takson Pinus sylvestris L. ormanında, 49 takson Carpinus orientalis Mill. ormanında ve 24 takson Pinus maritima Lam. ormanındadır. 


\section{Introduction}

Fungal species play important roles in ecosystems. For instance, they decompose organic materials and occupy diverse niches in forest ecosystems. In order to learn their ecological roles, it is necessary to determine their distribution areas, species diversity and the habitat types habitats they occupy. In this way, we can get useful information about common and widely distributed, rare, poisonous or edible species, or species that are important in terms of the ecological cycle. Such a knowledge helps mycologists to understand the macrofungal diversity of an area, region or even a country and allows to make a comparison with the macrofungal data of other studied places. In addition, it is also possible to reveal new or rare species in this way. During field studies, it is important to learn the knowledge of local people about mushrooms and to determine their ways to use them ethnomicologically.

Many studies on macrofungal diversity were carried out and yet many are still ongoing both in Turkey and in world. As a result of these studies, significant contributions have been made to the macrofungal diversity of Turkey. A checklist of the fungi of Turkey was published in 2020 with broad cooperation of Turkish mycologists (Sesli et al. 2020). According to this checklist, a total of 5865 fungal taxa, including 2782 Basidiomycota, 2728 Ascomycota 282 Myxomycota, 2 Chytridiomycota, 33 Oomycota and 38 Zygomycota species identified in Turkey have been listed so far. Regarding the ecology and habitat choices of these taxa, the majority are found in coniferous and broadleaved (latifolius) forest ecosystems. Other environments in which fungal species can be found were reported as meadows, waterfronts, humid areas and similar different habitats. When the relevant literature was reviewed, no study was found on fungal diversity of Samanlı Mountains. Samanlı Mountains has different kind of the forest types formed by various trees such as Abies sp., Carpinus sp., Fagus sp., Pinus sp. and Quercus sp. The climatic conditions of the mountain provide optimum growth of mushrooms. We therefore chose it as the study area to determine the macrofungal diversity present and contribute to the Turkish mycobiota.

\section{Materials and Methods}

\section{Description of the area}

Samanl1 Mountains are located in the southeast of the Marmara Region in Turkey (Fig. 1). The range stretches between Bozburun at the edge of Armutlu Peninsula in the west, and Geyve Strait formed by Sakarya River in the east. A close look at the natural vegetation of the study area highlights kermes oak (Quercus coccifera L.), holly oak (Quercus ilex L.), and bay laurel (Laurus nobilis L.) as the main shrubs and ligneous plants in the maquis formation up to 500-600m. Hawthorn (Crataegus oxyacantha L.) and a Black Sea enclave, boxwood (Buxus sempervirens L.), are seen in patches among maquis elements. The main ligneous plants in the forest cover of the area are pedunculate oak (Quercus pedunculata Ehrh.), oriental beech (Fagus orientalis Lipsky), Uludağ fir (Abies nordmanniana subsp. bornmuelleriana (Mattf.) Coode \& Cullen), chestnut (Castanea sativa Mill.), black pine (Pinus nigra subsp. caramanica (Loudon) Businský), stone pine (Pinus pinea L.), Turkish pine (Pinus brutia Ten.), common hornbeam (Carpinus betulus L.) and Scots pine (Pinus sylvestris Lour.). Groups of oriental planes (Platanus orientalis L.), maple (Acer platanoides L.), and white poplar (Populus alba L.) can also be seen in patches. The area is in the Mediterranean climatic zone in terms of macroclimatic type, and the annual rainfall varies between $400 \mathrm{~mm}$ and $1200 \mathrm{~mm}$.

\section{Collection and identification of the species}

The macrofungi specimens were collected from 148 localities in Bursa, Kocaeli, Sakarya and Yalova provinces during the years 2012-2015 (Fig. 1, Table 1). The localities are listed alphabetically, and coordinates, heights, habitats and collecting time were given in Table 1. Partition numbers refer to the numbers given to forest areas by the forest management directorates in Table 1 . Important macroscopical features and ecological information of the specimens were noted in the field and digital images were taken in their habitat. Collected specimens were dried in dehydrators after each study day and the dried materials were put into plastic bags to bring them to the fungarium in good condition for further analysis. Micromorphological characters were examined using a Leica DM3000 light microscope and photographed digitally. Specimen tissues were examined with some chemical reagents (Melzer; $\mathrm{KOH}$ in 10\%, 5\%, $3 \%$, or $2 \%$ solutions; cotton blue; IKI; etc.) for macroscopic and microscopic studies. The measurements of at least 20 spores per specimen were taken. The specimens were identified according to Eriksson \& Ryvarden (1973,1976), Eriksson et al. (1978, 1984), Moser (1983), Breitenbach \& Kränzlin (1984, 1986, 1991, 1995, 2000), Hjortstam et al. (1987, 1988), Candusso \& Lanzoni (1990), Ryvarden \& Gilbertson (1993, 1994), Candusso (1997), Basso (1999), Riva (2003a, 2003b), Galli (2003a, 2003b, 2004, 2006, 2007a, 2007b), Neville \& Poumarat (2004), Bernicchia (2005), Horak (2005), Muñoz (2005), Kränzlin (2005), Medardi (2006), Robich (2007), Parra (2008), Michael et. al (2014), Knudsen \& Vesterholt (2008) and Christensen \& Heilmann-Clausen (2013). New records were checked according to Sesli et al. (2020). Taxa, family, and author citations are quoted according to Cannon \& Kirk (2007), Kirk et al. (2008), Index Fungorum (http://www.indexfungorum.org/Names/Names.asp) and MycoBank (http://www.mycobank.org). The specimens are kept in the Fungarium of Mushroom Application and Research Centre, Selçuk University, Konya, Turkey. 


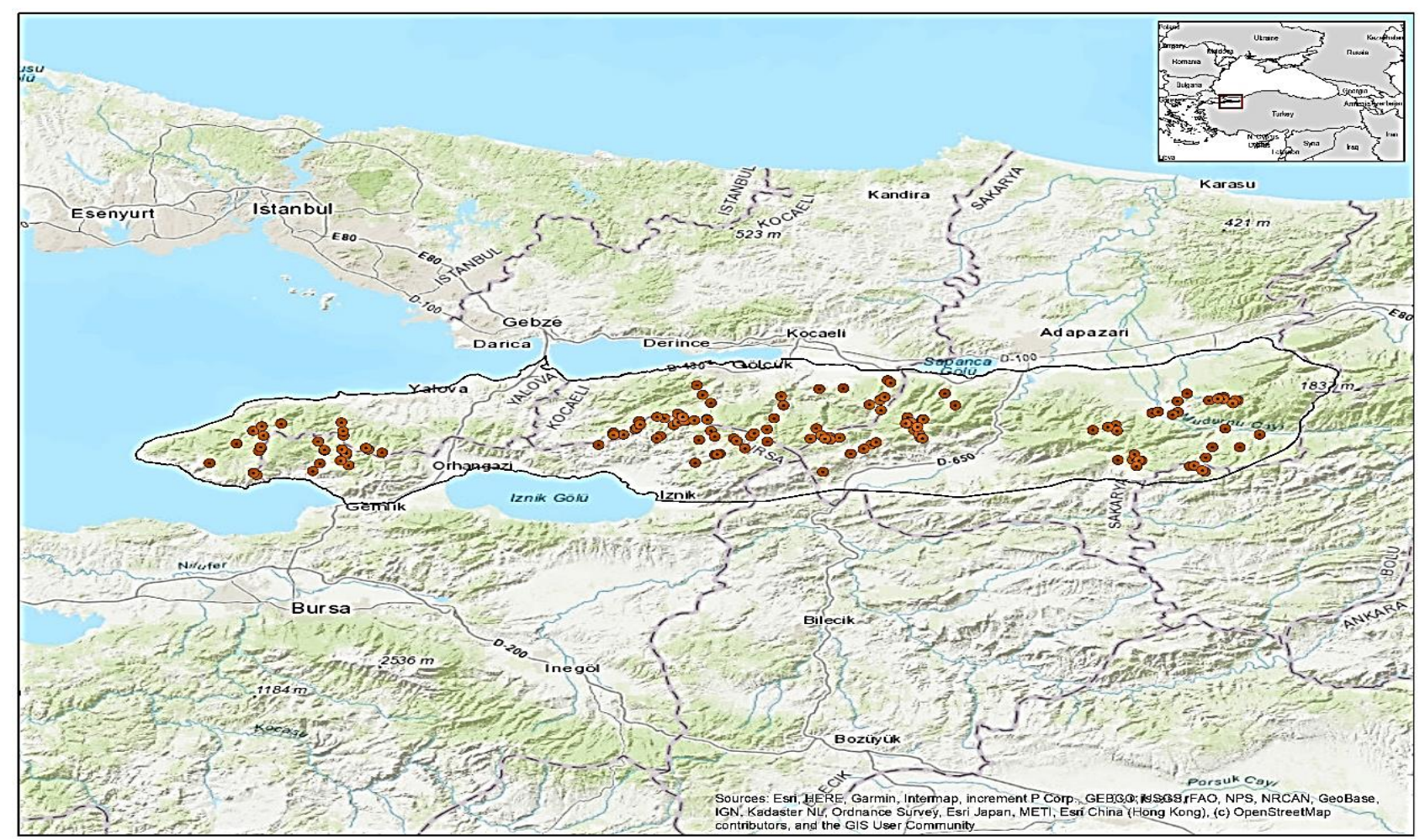

Fig. 1. Map showing the study area. The black line shows the borders of Samanl1 Mountains and solid coloured circles correspond the different localities where the specimens were collected.

\section{$\underline{\text { Locality List }}$}

Table 1. The locality names, coordinates, altitudes, habitat types and collection date details.

\begin{tabular}{|c|c|c|c|c|c|}
\hline Loc.No & Localities & Coordinates & Height & $\begin{array}{c}\text { Habitats } \\
\end{array}$ & Date \\
\hline L1 & Bursa, Gemlik, Fevziye Vill., Karagöl district & $\begin{array}{l}40^{\circ} 21^{\prime} 04^{\prime \prime} \mathrm{N} \\
29^{\circ} 18^{\prime} 26^{\prime \prime} \mathrm{E}\end{array}$ & $776 \mathrm{~m}$ & $\begin{array}{l}\text { F. orientalis, P. nigra, Quercus } \\
\text { sp. forest }\end{array}$ & 10.X.2014 \\
\hline L2 & $\begin{array}{l}\text { Bursa, Gemlik, Gemlik-Sarıkaya road, } \\
\text { Soğanlıtarla district }\end{array}$ & $\begin{array}{l}40^{\circ} 32^{\prime} 25^{\prime \prime} \mathrm{N} \\
29^{\circ} 11^{\prime} 50^{\prime \prime} \mathrm{E}\end{array}$ & $660 \mathrm{~m}$ & Quercus sp. forest & 07.VI.2013 \\
\hline $\mathbf{L 3}$ & $\begin{array}{l}\text { Bursa, Gemlik, Haydariye Vill., Çeşme } \\
\text { district }\end{array}$ & $\begin{array}{l}40^{\circ} 30^{\prime} 19^{\prime \prime} \mathrm{N} \\
29^{\circ} 07^{\prime} 07^{\prime \prime} \mathrm{E}\end{array}$ & $420 \mathrm{~m}$ & F. orientalis, $C$. orientalis forest & 02.VI.2014 \\
\hline L4 & $\begin{array}{l}\text { Bursa, Gemlik, Haydariye Vill., Dereiçi } \\
\text { district }\end{array}$ & $\begin{array}{l}40^{\circ} 32^{\prime} 27^{\prime \prime} \mathrm{N} \\
29^{\circ} 08^{\prime} 59^{\prime \prime} \mathrm{E}\end{array}$ & $470 \mathrm{~m}$ & F. orientalis, $C$. orientalis & 02.VI.2014 \\
\hline L5 & Bursa, Gemlik, Haydariye Vill., Dörtyol cross & $\begin{array}{l}40^{\circ} 30^{\prime} 59^{\prime \prime} \mathrm{N} \\
29^{\circ} 08^{\prime} 49^{\prime \prime} \mathrm{E}\end{array}$ & $605 \mathrm{~m}$ & Quercus sp. forest & 23.X.2013 \\
\hline L6 & Bursa, Gemlik, Haydariye Vill., Tokat district & $\begin{array}{l}40^{\circ} 32^{\prime} 22^{\prime \prime} \mathrm{N}, \\
29^{\circ} 07^{\prime} 09^{\prime \prime} \mathrm{E}\end{array}$ & $425 \mathrm{~m}$ & $\begin{array}{l}F \text {. orientalis, } C \text {. orientalis, } \\
\text { Quercus sp., } R \text {. ponticum forest }\end{array}$ & 03.VI.2013 \\
\hline L7 & $\begin{array}{l}\text { Bursa, Gemlik, Haydariye Vill., upper parts } \\
\text { of a gezintiyolu district }\end{array}$ & $\begin{array}{l}40^{\circ} 30^{\prime} 37^{\prime \prime} \mathrm{N} \\
29^{\circ} 06^{\prime} 37^{\prime \prime} \mathrm{E}\end{array}$ & $605 \mathrm{~m}$ & P. nigra, A. unedo forest & 23.X.2013 \\
\hline L8 & $\begin{array}{l}\text { Bursa, Gemlik, Haydariye Vill., Yeşilbaştepe } \\
\text { gezintiyolu district }\end{array}$ & $\begin{array}{l}40^{\circ} 30^{\prime} 12^{\prime \prime} \mathrm{N} \\
29^{\circ} 06^{\prime} 53^{\prime \prime} \mathrm{E}\end{array}$ & $405 \mathrm{~m}$ & Quercus sp. forest & 02.VI.2014 \\
\hline L9 & $\begin{array}{l}\text { Bursa, Gemlik, Küçükkum, upward of } \\
\text { Gendarme station }\end{array}$ & $\begin{array}{l}40^{\circ} 27^{\prime} 48^{\prime \prime} \mathrm{N} \\
29^{\circ} 07^{\prime} 47^{\prime \prime} \mathrm{E}\end{array}$ & $300 \mathrm{~m}$ & P. nigra forest & 20.XI.2013 \\
\hline L10 & Bursa, Gemlik, Narlı Vill. & $\begin{array}{l}40^{\circ} 29^{\prime} 26^{\prime \prime} \mathrm{N} \\
28^{\circ} 59^{\prime} 27^{\prime \prime} \mathrm{E}\end{array}$ & $450 \mathrm{~m}$ & Quercus sp. forest & 02.VI.2014 \\
\hline L11 & $\begin{array}{l}\text { Bursa, Gemlik, opposite to partition no: } 250 \\
\text { of Haydariye Vill. }\end{array}$ & $\begin{array}{l}40^{\circ} 30^{\prime} 27^{\prime \prime} \mathrm{N} \\
29^{\circ} 09^{\prime} 44 " \mathrm{E}\end{array}$ & $740 \mathrm{~m}$ & $\begin{array}{l}F \text {. orientalis, } C \text {. orientalis, } \\
\text { Quercus sp., } R \text {. ponticum forest }\end{array}$ & 03.VI.2013 \\
\hline L12 & Bursa, Gemlik, partition no 44 , & $\begin{array}{l}40^{\circ} 34^{\prime} 14^{\prime \prime} \mathrm{N} \\
29^{\circ} 09^{\prime} 10^{\prime \prime} \mathrm{E}\end{array}$ & $557 \mathrm{~m}$ & F. orientalis forest & 26.X.2013 \\
\hline L13 & Bursa, Gemlik, upward of Haydariye Vill. & $\begin{array}{l}40^{\circ} 31^{\prime} 02^{\prime \prime} \mathrm{N} \\
29^{\circ} 08^{\prime} 51^{\prime \prime} \mathrm{E}\end{array}$ & $40 \mathrm{~m}$ & $\begin{array}{l}F \text {. orientalis, } \text { C. orientalis, } \\
\text { Quercus sp., } R \text {. ponticum forest }\end{array}$ & 03.VI.2013 \\
\hline L14 & Bursa, Gemlik, upward of Narlı Vill. & $\begin{array}{l}40^{\circ} 29^{\prime} 01^{\prime \prime} \mathrm{N}, \\
28^{\circ} 59^{\prime} 31^{\prime \prime} \mathrm{E} \\
\end{array}$ & $480 \mathrm{~m}$ & $P$. pinea forest & 20.XI.2013 \\
\hline
\end{tabular}


Table 1 Continued.

\begin{tabular}{|c|c|c|c|c|c|}
\hline L15 & Bursa, Haydariye Vill., Kolaçandere district & $\begin{array}{l}40^{\circ} 31^{\circ} 59^{\prime \prime} \mathrm{N} \\
29^{\circ} 09^{\prime} 29^{\prime \prime} \mathrm{E}\end{array}$ & $544 \mathrm{~m}$ & F. orientalis forest & 23.X.2013 \\
\hline L16 & Bursa, İznik, Aybaşı district, forest & $\begin{array}{l}40^{\circ} 36^{\prime} 39^{\prime \prime} \mathrm{N} \\
29^{\circ} 42^{\prime} 57^{\prime \prime} \mathrm{E}\end{array}$ & $950 \mathrm{~m}$ & Quercus sp. & 24.X.2013 \\
\hline L17 & Bursa, İznik, Çandarlı series, partition no 20 & $\begin{array}{l}40^{\circ} 34^{\prime} 13^{\prime \prime N} \\
29^{\circ} 53^{\prime} 06^{\prime \prime} \mathrm{E}\end{array}$ & $955 \mathrm{~m}$ & F. orientalis forest & 05.VI.2013 \\
\hline L18 & Bursa, İznik, Çandarlı Vill. & $\begin{array}{l}40^{\circ} 31^{\prime} 45^{\prime \prime} \mathrm{N} \\
29^{\circ} 49^{\prime} 13^{\prime \prime} \mathrm{E}\end{array}$ & $858 \mathrm{~m}$ & P. sylvestris, Quercus sp. forest & 25.X.2013 \\
\hline L19 & Bursa, İznik, Çandarlı, Sarıçam district & $\begin{array}{l}40^{\circ} 31^{\prime} 49^{\prime \prime} \mathrm{N} \\
29^{\circ} 49^{\prime} 25^{\prime \prime} \mathrm{E}\end{array}$ & $934 \mathrm{~m}$ & P. sylvestris forest & 05.XI.2015 \\
\hline L20 & Bursa, İznik, Çandarlı, Subatım district & $\begin{array}{l}40^{\circ} 34^{\prime} 32^{\prime \prime} \mathrm{N} \\
29^{\circ} 53^{\prime} 25^{\prime \prime} \mathrm{E}\end{array}$ & $990 \mathrm{~m}$ & F. orientalis forest & 05.VI.2013 \\
\hline L21 & $\begin{array}{l}\text { Bursa, İznik, downward of Mecidiye Vill., } \\
\text { Boğazdere district }\end{array}$ & $\begin{array}{l}40^{\circ} 34^{\prime} 42^{\prime \prime} \mathrm{N} \\
29^{\circ} 44^{\prime} 52^{\prime \prime} \mathrm{E}\end{array}$ & $623 \mathrm{~m}$ & Quercus sp. forest & 24.X.2013 \\
\hline $\mathbf{L 2 2}$ & $\begin{array}{l}\text { Bursa, İznik, Elmalı Vill., upward of the Paşa } \\
\text { neighbourhood }\end{array}$ & $\begin{array}{l}40^{\circ} 32^{\prime} 34^{\prime \prime} \mathrm{N} \\
29^{\circ} 52^{\prime} 25^{\prime \prime} \mathrm{E}\end{array}$ & $860 \mathrm{~m}$ & F. orientalis forest & 05.VI.2013 \\
\hline $\mathbf{L 2 3}$ & Bursa, İznik, Hakkıdüzlüğü district & $\begin{array}{l}40^{\circ} 33^{\prime} 41^{\prime \prime} \mathrm{N} \\
29^{\circ} 47^{\prime} 31^{\prime \prime} \mathrm{E}\end{array}$ & $850 \mathrm{~m}$ & P. nigra, Quercus sp. forest & 25.X.2013 \\
\hline L24 & Bursa, İznik, İznik-Gölcük border & $\begin{array}{l}40^{\circ} 36^{\prime} 23^{\prime \prime} \mathrm{N} \\
29^{\circ} 45^{\prime} 26^{\prime \prime} \mathrm{E}\end{array}$ & $921 \mathrm{~m}$ & F. orientalis forest & 11.X.2014 \\
\hline L25 & Bursa, İznik, Kırıntı & $\begin{array}{l}40^{\circ} 333^{\prime} 33^{\prime \prime} \mathrm{N} \\
29^{\circ} 51^{\prime} 34^{\prime \prime} \mathrm{E}\end{array}$ & $886 \mathrm{~m}$ & $\begin{array}{l}\text { P. sylvestris, Quercus sp., } C \text {. } \\
\text { orientalis forest }\end{array}$ & 11.X.2014 \\
\hline L26 & $\begin{array}{l}\text { Bursa, İznik, Merkeztepe district, partition no } \\
27\end{array}$ & $\begin{array}{l}40^{\circ} 333^{\prime} 29^{\prime \prime} \mathrm{N} \\
29^{\circ} 54^{\prime} 50^{\prime \prime} \mathrm{E}\end{array}$ & $940 \mathrm{~m}$ & F. orientalis forest & 05.VI.2013 \\
\hline L27 & Bursa, İznik, next to a mine & $\begin{array}{l}40^{\circ} 36^{\prime} 05^{\prime \prime} \mathrm{N} \\
29^{\circ} 46^{\prime} 01 " \mathrm{E}\end{array}$ & $950 \mathrm{~m}$ & P. nigra, Quercus sp. forest & 25.X.2013 \\
\hline L28 & Bursa, İznik, Pilavtepe district & $\begin{array}{l}40^{\circ} 35^{\prime} 04^{\prime \prime} \mathrm{N} \\
29^{\circ} 40^{\prime} 38^{\prime \prime} \mathrm{E}\end{array}$ & $730 \mathrm{~m}$ & Quercus sp. forest & 04.VI.2013 \\
\hline $\mathbf{L 2 9}$ & Bursa, İznik, Pilavtepe district & $\begin{array}{l}40^{\circ} 36^{\prime} 46^{\prime \prime} \mathrm{N} \\
29^{\circ} 42^{\prime} 48^{\prime \prime} \mathrm{E}\end{array}$ & $710 \mathrm{~m}$ & Quercus forest & 24.X.2013 \\
\hline L30 & Bursa, İznik, upward of Hacıosman Vill. & $\begin{array}{l}40^{\circ} 36^{\prime} 22^{\prime \prime} \mathrm{N} \\
29^{\circ} 48^{\prime} 20^{\prime \prime} \mathrm{E}\end{array}$ & $839 \mathrm{~m}$ & $\begin{array}{l}\text { Quercus sp., } F \text {. orientalis, } P . \\
\text { sylvestris forest }\end{array}$ & 11.X.2014 \\
\hline L31 & $\begin{array}{l}\text { Bursa, İznik, upward of Kahraman } \\
\text { neighbourhood }\end{array}$ & $\begin{array}{l}40^{\circ} 35^{\prime} 59^{\prime \prime} \mathrm{N} \\
29^{\circ} 45^{\prime} 14^{\prime \prime} \mathrm{E}\end{array}$ & $740 \mathrm{~m}$ & P. nigra, Quercus sp. forest & 25.X.2013 \\
\hline L32 & $\begin{array}{l}\text { Bursa, Mahmudiye, Haciosman meadow- } \\
\text { Kutluca crossroads }\end{array}$ & $\begin{array}{l}40^{\circ} 34^{\prime} 04^{\prime \prime} \mathrm{N} \\
29^{\circ} 49^{\prime} 11 " \mathrm{E}\end{array}$ & $995 \mathrm{~m}$ & F. orientalis, Quercus sp. forest & 04.VI.2014 \\
\hline L33 & Bursa, Mahmudiye, Kutluca Vill. & $\begin{array}{l}40^{\circ} 33^{\prime} 51^{\prime \prime} \mathrm{N} \\
29^{\circ} 51^{\prime} 13^{\prime \prime} \mathrm{E}\end{array}$ & $850 \mathrm{~m}$ & F. orientalis, $P$. sylvestris forest & 04.VI.2014 \\
\hline L34 & Bursa, Mahmudiye, Taşlıtarla district & $\begin{array}{l}40^{\circ} 36^{\prime} 09^{\prime \prime} \mathrm{N} \\
29^{\circ} 46^{\prime} 04^{\prime \prime} \mathrm{E}\end{array}$ & $947 \mathrm{~m}$ & $F$. orientalis, $C$. orientalis forest & 04.VI.2013 \\
\hline L35 & Bursa, Mahmudiye, Yapraklıdere district & $\begin{array}{l}39^{\circ} 55^{\prime} 07^{\prime \prime} \mathrm{N} \\
29^{\circ} 43^{\prime} 41^{\prime \prime} \mathrm{E}\end{array}$ & $919 \mathrm{~m}$ & F. orientalis, $C$. orientalis forest & 04.VI.2013 \\
\hline L36 & Bursa, Mahmudiye, Yoncalık district & $\begin{array}{l}40^{\circ} 34^{\prime} 55^{\prime \prime} \mathrm{N} \\
29^{\circ} 48^{\prime} 49^{\prime \prime} \mathrm{E}\end{array}$ & $1005 \mathrm{~m}$ & F. orientalis, C. orientalis forest & 04.VI.2013 \\
\hline L37 & Kocaeli, Gölcük, Başkiraz Plateau & $\begin{array}{l}40^{\circ} 36^{\prime} 05^{\prime \prime} \mathrm{N} \\
29^{\circ} 41^{\prime} 02^{\prime \prime} \mathrm{E}\end{array}$ & $780 \mathrm{~m}$ & Quercus sp. forest & 31.V.2014 \\
\hline L38 & $\begin{array}{l}\text { Kocaeli, Gölcük, downward of Cansuyu } \\
\text { district }\end{array}$ & $\begin{array}{l}40^{\circ} 36^{\prime} 22^{\prime \prime} \mathrm{N} \\
29^{\circ} 48^{\prime} 20^{\prime \prime} \mathrm{E}\end{array}$ & $865 \mathrm{~m}$ & F. orientalis forest & 26.X.2014 \\
\hline L39 & $\begin{array}{l}\text { Kocaeli: Gölcük, İhsaniye Vill., Kurtlarvadisi } \\
\text { district }\end{array}$ & $\begin{array}{l}40^{\circ} 38^{\prime} 25^{\prime \prime} \mathrm{N} \\
29^{\circ} 48^{\prime} 46^{\prime \prime} \mathrm{E}\end{array}$ & $250 \mathrm{~m}$ & F. orientalis, Quercus sp. forest & 26.X.2014 \\
\hline L40 & $\begin{array}{l}\text { Kocaeli, Gölcük, İhsaniye, Ayvazpınarı } \\
\text { district, picnic area }\end{array}$ & $\begin{array}{l}40^{\circ} 36^{\prime} 55^{\prime \prime} \mathrm{N} \\
29^{\circ} 44^{\prime} 56^{\prime \prime} \mathrm{E}\end{array}$ & $830 \mathrm{~m}$ & F. orientalis forest & 31.V.2014 \\
\hline L41 & $\begin{array}{l}\text { Kocaeli, Gölcük, İhsaniye, Ayvazpınarı } \\
\text { district, downward of picnic area }\end{array}$ & $\begin{array}{l}40^{\circ} 37^{\prime} 02^{\prime \prime} \mathrm{N} \\
29^{\circ} 45^{\prime} 11 " \mathrm{E}\end{array}$ & $780 \mathrm{~m}$ & $\begin{array}{l}\text { F. orientalis, } C \text {. orientalis, } C \text {. } \\
\text { sativa, } C \text {. avellana forest }\end{array}$ & 26.X.2014 \\
\hline $\mathbf{L 4 2}$ & Kocaeli, Gölcük, Mecidiye Vill. & $\begin{array}{l}40^{\circ} 35^{\prime} 13^{\prime \prime} \mathrm{N} \\
29^{\circ} 44^{\prime} 56^{\prime \prime} \mathrm{E}\end{array}$ & $760 \mathrm{~m}$ & Quercus sp. forest & 31.V.2014 \\
\hline L43 & Kocaeli, Gölcük, Menekşe Plateau & $\begin{array}{l}40^{\circ} 35^{\prime} 01^{\prime \prime} \mathrm{N} \\
29^{\circ} 54^{\prime} 48^{\prime \prime} \mathrm{E}\end{array}$ & $890 \mathrm{~m}$ & F. orientalis forest & 05.VI.2013 \\
\hline L44 & Kocaeli, Gölcük, next to İnci taşocağ1 district & $\begin{array}{l}40^{\circ} 36^{\prime} 06^{\prime \prime} \mathrm{N} \\
29^{\circ} 46^{\prime} 50^{\prime \prime} \mathrm{E}\end{array}$ & $922 \mathrm{~m}$ & F. orientalis forest & 31.V.2014 \\
\hline $\mathbf{L 4 5}$ & $\begin{array}{l}\text { Kocaeli, Gölcük, on the way of Ayvazpınarı } \\
\text { district }\end{array}$ & $\begin{array}{l}40^{\circ} 36^{\prime} 50^{\prime \prime} \mathrm{N} \\
29^{\circ} 45^{\prime} 26^{\prime \prime} \mathrm{E}\end{array}$ & $840 \mathrm{~m}$ & $\begin{array}{l}\text { F. orientalis, C. orientalis, } \\
\text { Quercus sp. forest }\end{array}$ & 01.X.2014 \\
\hline L46 & $\begin{array}{l}\text { Kocaeli, Gölcük, on the way of Eriklitepe } \\
\text { district }\end{array}$ & $\begin{array}{l}40^{\circ} 36^{\prime} 08^{\prime \prime} \mathrm{N} \\
29^{\circ} 45^{\prime} 55^{\prime \prime} \mathrm{E}\end{array}$ & $970 \mathrm{~m}$ & F. orientalis forest & 31.V.2014 \\
\hline
\end{tabular}


Table 1 Continued.

\begin{tabular}{|c|c|c|c|c|c|}
\hline L47 & $\begin{array}{l}\text { Kocaeli, Gölcük, on the way of İnci taşocağ } 1 \\
\text { district to Gölcük, } 1 . \mathrm{km} \text { down of Şelale district }\end{array}$ & $\begin{array}{l}40^{\circ} 36^{\prime} 22^{\prime \prime} \mathrm{N} \\
29^{\circ} 48^{\prime} 20^{\prime \prime} \mathrm{E}\end{array}$ & $850 \mathrm{~m}$ & C. orientalis forest & 01.X.2014 \\
\hline L48 & Kocaeli, Gölcük, Pilavtepe crossroad & $\begin{array}{l}40^{\circ} 35^{\prime} 38^{\prime \prime} \mathrm{N} \\
29^{\circ} 41^{\prime} 08^{\prime \prime} \mathrm{E}\end{array}$ & $760 \mathrm{~m}$ & P. nigra, Quercus sp. forest & 01.X.2014 \\
\hline L49 & $\begin{array}{l}\text { Kocaeli, Gölcük, upward of Mecidiye Vill., } \\
\text { Kestanelik district }\end{array}$ & $\begin{array}{l}40^{\circ} 39^{\prime} 27^{\prime \prime} \mathrm{N} \\
29^{\circ} 47^{\prime} 52^{\prime \prime} \mathrm{E}\end{array}$ & $560 \mathrm{~m}$ & C. sativa, Quercus sp. forest & 01.X.2014 \\
\hline L50 & Kocaeli, Karamürsel, exit of Tahtalı Vill. & $\begin{array}{l}40^{\circ} 34^{\prime} 21 " \mathrm{~N} \\
29^{\circ} 39^{\prime} 20^{\prime \prime} \mathrm{E}\end{array}$ & $730 \mathrm{~m}$ & Quercus sp. forest & 01.VI.2014 \\
\hline L51 & Kocaeli, Karamürsel, Fulacık crossroad & $\begin{array}{l}40^{\circ} 34^{\prime} 37^{\prime \prime} \mathrm{N} \\
29^{\circ} 38^{\prime} 16^{\prime \prime} \mathrm{E}\end{array}$ & $685 \mathrm{~m}$ & meadow area & 01.X.2014 \\
\hline $\mathbf{L 5 2}$ & $\begin{array}{l}\text { Kocaeli, Karamürsel, Fulacık, exit from } \\
\text { Tahtalı Vill., next to the fountain }\end{array}$ & $\begin{array}{l}40^{\circ} 34^{\prime} 18^{\prime \prime} \mathrm{N} \\
29^{\circ} 38^{\prime} 16^{\prime \prime} \mathrm{E}\end{array}$ & $670 \mathrm{~m}$ & $\begin{array}{l}\text { F. orientalis, } C \text {. sativa, } C \text {. } \\
\text { orientalis forest }\end{array}$ & 01.X.2014 \\
\hline $\mathbf{L 5 3}$ & Kocaeli, Karamürsel, Fulacık Vill., & $\begin{array}{l}40^{\circ} 36^{\prime} 06^{\prime \prime} \mathrm{N} \\
29^{\circ} 46^{\prime} 50^{\prime \prime} \mathrm{E}\end{array}$ & $922 \mathrm{~m}$ & Quercus sp. forest & 01.VI.2014 \\
\hline L54 & $\begin{array}{l}\text { Kocaeli, Karamürsel, Mahmudiye Vill., } \\
\text { Tahtalı roadside }\end{array}$ & $\begin{array}{l}40^{\circ} 31^{\prime} 17^{\prime \prime} \mathrm{N} \\
29^{\circ} 38^{\prime} 15^{\prime \prime} \mathrm{E}\end{array}$ & $690 \mathrm{~m}$ & $F$. orientalis, $P$. nigra forest & 24.X.2013 \\
\hline L55 & Kocaeli, Maşukiye, across Sislivadi district & $\begin{array}{l}40^{\circ} 39^{\prime} 14^{\prime \prime} \mathrm{N} \\
30^{\circ} 07^{\prime} 45^{\prime \prime} \mathrm{E}\end{array}$ & $1200 \mathrm{~m}$ & $\begin{array}{l}\text { A. nordmanniana subsp. } \\
\text { bornmuelleriana, F. orientalis } \\
\text { forest }\end{array}$ & 25.X.2014 \\
\hline L56 & $\begin{array}{l}\text { Kocaeli, Maşukiye, entrance of Kuzuyayla } \\
\text { Nature Park }\end{array}$ & $\begin{array}{l}40^{\circ} 38^{\prime} 50^{\prime \prime} \mathrm{N} \\
30^{\circ} 06^{\prime} 53^{\prime \prime} \mathrm{E}\end{array}$ & $1400 \mathrm{~m}$ & $\begin{array}{l}\text { A. nordmanniana subsp. } \\
\text { bornmuelleriana, F. orientalis } \\
\text { forest }\end{array}$ & 25.X.2014 \\
\hline L57 & $\begin{array}{l}\text { Kocaeli, Maşukiye, Kartepe road, gezintiyolu } \\
\text { district }\end{array}$ & $\begin{array}{l}40^{\circ} 41^{\prime} 00^{\prime \prime} \mathrm{N} \\
30^{\circ} 08^{\prime} 59^{\prime \prime} \mathrm{E}\end{array}$ & $460 \mathrm{~m}$ & $F$. orientalis, $P$. nigra forest & 28.IX.2014 \\
\hline L58 & Kocaeli, Maşukiye, Kartepe, Altıoluk Plateau & $\begin{array}{l}40^{\circ} 37^{\prime} 28^{\prime \prime} \mathrm{N} \\
30^{\circ} 06^{\prime} 59^{\prime \prime} \mathrm{E}\end{array}$ & $1310 \mathrm{~m}$ & F. orientalis forest & 25.X.2014 \\
\hline L59 & $\begin{array}{l}\text { Kocaeli: Suadiye, Altioluk Plateau, the back } \\
\text { of the transmitter }\end{array}$ & $\begin{array}{l}40^{\circ} 38^{\prime} 12^{\prime \prime} \mathrm{N} \\
30^{\circ} 05^{\prime} 52^{\prime \prime} \mathrm{E}\end{array}$ & $1360 \mathrm{~m}$ & F. orientalis forest & 27.V.2014 \\
\hline L60 & Kocaeli, Suadiye, Hafizıntarlası district, & $\begin{array}{l}40^{\circ} 40^{\prime} 16^{\prime \prime} \mathrm{N} \\
30^{\circ} 00^{\prime} 27^{\prime \prime} \mathrm{E}\end{array}$ & $400 \mathrm{~m}$ & $\begin{array}{l}\text { Quercus sp., F. orientalis, } C \text {. } \\
\text { orientalis, C. avelleana forest }\end{array}$ & 27.XI.2012 \\
\hline L61 & Kocaeli, Suadiye, on Kartepe road, left side & $\begin{array}{l}40^{\circ} 40^{\prime} 20^{\prime \prime} \mathrm{N} \\
30^{\circ} 03^{\prime} 04^{\prime \prime} \mathrm{E}\end{array}$ & $540 \mathrm{~m}$ & P. sylvestris forest & 27.XI.2012 \\
\hline L62 & $\begin{array}{l}\text { Kocaeli, Yuvacik, across Servetiye, Dikkulak } \\
\text { district }\end{array}$ & $\begin{array}{l}40^{\circ} 39^{\prime} 18^{\prime \prime} \mathrm{N} \\
29^{\circ} 56^{\prime} 22^{\prime \prime} \mathrm{E}\end{array}$ & $460 \mathrm{~m}$ & $\begin{array}{l}\text { F. orientalis, } C \text {. orientalis, } C \text {. } \\
\text { sativa forest }\end{array}$ & 17.IV.2013 \\
\hline L63 & Kocaeli, Yuvacık, Aytepe district & $\begin{array}{l}40^{\circ} 36^{\prime} 30^{\prime \prime} \mathrm{N} \\
29^{\circ} 55^{\prime} 36^{\prime \prime} \mathrm{E}\end{array}$ & $960 \mathrm{~m}$ & $\begin{array}{l}\text { F. orientalis, } C . \text { sativa, } C . \\
\text { orientalis forest }\end{array}$ & 28.XI.2012 \\
\hline L64 & Kocaeli, Yuvacık, entrance of İnönü Plateau & $\begin{array}{l}40^{\circ} 35^{\prime} 09^{\prime \prime} \mathrm{N} \\
30^{\circ} 00^{\prime} 06^{\prime \prime} \mathrm{E}\end{array}$ & $1240 \mathrm{~m}$ & $\begin{array}{l}\text { P. sylvestris, A. nordmanniana } \\
\text { subsp. bornmuelleriana, } F \text {. } \\
\text { orientalis forest }\end{array}$ & 28.XI.2012 \\
\hline L65 & Kocaeli, Yuvacık, İnönü Plateau & $\begin{array}{l}40^{\circ} 33^{\prime} 52^{\prime \prime} \mathrm{N} \\
29^{\circ} 59^{\prime} 30^{\prime \prime} \mathrm{E}\end{array}$ & $1240 \mathrm{~m}$ & $\begin{array}{l}\text { A. nordmanniana subsp. } \\
\text { bornmuelleriana, } P \text {. nigra, } F \text {. } \\
\text { orientalis, } C \text {. orientalis, } R \text {. } \\
\text { ponticum forest }\end{array}$ & 29.IV.2014 \\
\hline L66 & $\begin{array}{l}\text { Kocaeli, Yuvacık, İnönü Plateau, Şehitlik } \\
\text { district road }\end{array}$ & $\begin{array}{l}40^{\circ} 33^{\prime} 58^{\prime \prime} \mathrm{N} \\
30^{\circ} 01^{\prime} 34^{\prime \prime} \mathrm{E}\end{array}$ & $1160 \mathrm{~m}$ & $F$. orientalis, $P$. sylvestris, forest & 09.VI.2013 \\
\hline L67 & $\begin{array}{l}\text { Kocaeli, Yuvacık, İnönü Plateau, Şehitlik } \\
\text { district }\end{array}$ & $\begin{array}{l}40^{\circ} 33 ' 58^{\prime \prime} \mathrm{N} \\
30^{\circ} 02^{\prime} 39^{\prime \prime} \mathrm{E}\end{array}$ & $1150 \mathrm{~m}$ & F. orientalis forest & 09.VI.2013 \\
\hline L68 & Kocaeli, Yuvacık, Servetiye mosque, roadside & $\begin{array}{l}40^{\circ} 38^{\prime} 09^{\prime \prime} \mathrm{N} \\
29^{\circ} 56^{\prime} 37^{\prime \prime} \mathrm{E}\end{array}$ & $450 \mathrm{~m}$ & $\begin{array}{l}F . \text { orientalis, } C \text {. sativa, } C . \\
\text { orientalis, } R \text {. ponticum forest }\end{array}$ & 17.IV.2013 \\
\hline L69 & Sakarya, Akyazı, Avcıçimeni district & $\begin{array}{l}40^{\circ} 31^{\prime} 05^{\prime \prime} \mathrm{N} \\
30^{\circ} 34^{\prime} 16^{\prime \prime} \mathrm{E}\end{array}$ & $1260 \mathrm{~m}$ & $\begin{array}{l}\text { A. nordmanniana subsp. } \\
\text { bornmuelleriana, F. orientalis, } \\
\text { Quercus sp. forest }\end{array}$ & 01.XI.2013 \\
\hline L70 & $\begin{array}{l}\text { Sakarya, Akyazı, between Avcıçimeni and } \\
\text { Yılanlıkaya district }\end{array}$ & $\begin{array}{l}40^{\circ} 31^{\prime} 02^{\prime \prime} \mathrm{N} \\
30^{\circ} 34^{\prime} 28^{\prime \prime} \mathrm{E}\end{array}$ & $1253 \mathrm{~m}$ & $\begin{array}{l}\text { A. nordmanniana subsp. } \\
\text { bornmuelleriana, F. orientalis } \\
\text { forest }\end{array}$ & 30.IX.2014 \\
\hline L71 & Sakarya, Akyazı, Çiğdem Plateau, & $\begin{array}{l}40^{\circ} 38^{\prime} 56^{\prime \prime} \mathrm{N} \\
30^{\circ} 52^{\prime} 13^{\prime \prime} \mathrm{E}\end{array}$ & $1460 \mathrm{~m}$ & $\begin{array}{l}\text { A. nordmanniana subsp. } \\
\text { bornmuelleriana forest }\end{array}$ & 24.V.2014 \\
\hline L72 & Sakarya, Akyazı, Dokumacı district, & $\begin{array}{l}40^{\circ} 33^{\prime} 08^{\prime \prime} \mathrm{N} \\
30^{\circ} 34^{\prime} 13^{\prime \prime} \mathrm{E}\end{array}$ & $1185 \mathrm{~m}$ & $\begin{array}{l}\text { A. nordmanniana subsp. } \\
\text { bornmuelleriana, C. orientalis, } \\
\text { Pteridium sp. forest }\end{array}$ & 02.XI.2012 \\
\hline L73 & $\begin{array}{l}\text { Sakarya, Akyaz1, Dokurcun, down part of } \\
\text { Dikmentepe district }\end{array}$ & $\begin{array}{l}40^{\circ} 39^{\prime} 03^{\prime \prime} \mathrm{N} \\
30^{\circ} 53^{\prime} 28^{\prime \prime} \mathrm{E}\end{array}$ & $1350 \mathrm{~m}$ & $\begin{array}{l}\text { A. nordmanniana subsp. } \\
\text { bornmuelleriana forest }\end{array}$ & 24.V.2014 \\
\hline L74 & $\begin{array}{l}\text { Sakarya, Akyazı, Dokurcun, Güldürüksu } \\
\text { district }\end{array}$ & $\begin{array}{l}40^{\circ} 38^{\prime} 41 " \mathrm{~N} \\
30^{\circ} 53^{\prime} 47^{\prime \prime} \mathrm{E}\end{array}$ & $1390 \mathrm{~m}$ & $\begin{array}{l}\text { A. nordmanniana subsp. } \\
\text { bornmuelleriana forest }\end{array}$ & 24.V.2014 \\
\hline
\end{tabular}


Table 1 Continued.

\begin{tabular}{|c|c|c|c|c|c|}
\hline L75 & Sakarya, Akyazı, Dokurcun, Kındıra Plateau & $\begin{array}{l}40^{\circ} 38^{\prime} 01 " \mathrm{~N} \\
30^{\circ} 49^{\prime} 12^{\prime \prime} \mathrm{E}\end{array}$ & $1390 \mathrm{~m}$ & $\begin{array}{l}\text { A. nordmanniana subsp. } \\
\text { bornmuelleriana, F. orientalis } \\
\text { forest }\end{array}$ & 24.V.2014 \\
\hline L76 & $\begin{array}{l}\text { Sakarya, Akyazı, Dokurcun, upward of } \\
\text { Güldürüksu district }\end{array}$ & $\begin{array}{l}40^{\circ} 37 ' 58^{\prime \prime N} \\
30^{\circ} 52^{\prime} 13^{\prime \prime} \mathrm{E}\end{array}$ & $1510 \mathrm{~m}$ & $\begin{array}{l}\text { A. nordmanniana subsp. } \\
\text { bornmuelleriana forest }\end{array}$ & 24.V.2014 \\
\hline L77 & $\begin{array}{l}\text { Sakarya, Akyazı, down part of Hardamalık, } \\
\text { Durmuşlar district }\end{array}$ & $\begin{array}{l}40^{\circ} 34^{\prime} 88^{\prime \prime} \mathrm{N}, \\
30^{\circ} 44^{\prime} 78^{\prime \prime} \mathrm{E}\end{array}$ & $203 \mathrm{~m}$ & $\begin{array}{l}\text { A. nordmanniana subsp. } \\
\text { bornmuelleriana forest }\end{array}$ & 22.V.2014 \\
\hline L78 & $\begin{array}{l}\text { Sakarya, Akyazı, Göktepe, Ahmediye Vill., } \\
\text { Kestanedüzü district }\end{array}$ & $\begin{array}{l}40^{\circ} 35^{\prime} 37^{\prime \prime} \mathrm{N} \\
30^{\circ} 32^{\prime} 26^{\prime \prime} \mathrm{E}\end{array}$ & $961 \mathrm{~m}$ & C. orientalis forest & 02.XI.2012 \\
\hline L79 & Sakarya, Akyazı, Isırganlık district & $\begin{array}{l}40^{\circ} 39^{\prime} 11 " \mathrm{~N}, \\
30^{\circ} 44^{\prime} 04^{\prime \prime} \mathrm{E}\end{array}$ & $1200 \mathrm{~m}$ & $\begin{array}{l}\text { A. nordmanniana subsp. } \\
\text { bornmuelleriana forest }\end{array}$ & 02.XI.2013 \\
\hline L80 & Sakarya, Akyazı, Kayabaşı, Kiremitlik district & $\begin{array}{l}40^{\circ} 32^{\prime} 43^{\prime \prime N} \\
30^{\circ} 42^{\prime} 51^{\prime \prime} \mathrm{E}\end{array}$ & $960 \mathrm{~m}$ & $\begin{array}{l}\text { A. nordmanniana subsp. } \\
\text { bornmuelleriana, } F \text {. orientalis } \\
\text { forest }\end{array}$ & 29.X.2014 \\
\hline $\mathbf{L 8 1}$ & Sakarya, Akyazı, Keremali Pateau & $\begin{array}{l}40^{\circ} 38^{\prime} 46^{\prime \prime} \mathrm{N} \\
30^{\circ} 45^{\prime} 34^{\prime \prime} \mathrm{E}\end{array}$ & $1100 \mathrm{~m}$ & $\begin{array}{l}\text { A. nordmanniana subsp. } \\
\text { bornmuelleriana, } P \text {. sylvestris, } R \text {. } \\
\text { ponticum forest }\end{array}$ & 03.XI.2012 \\
\hline L82 & $\begin{array}{l}\text { Sakarya, Akyazı, Keremali Plateau, behind } \\
\text { the Mosque }\end{array}$ & $\begin{array}{l}40^{\circ} 37^{\prime} 46^{\prime \prime} \mathrm{N} \\
30^{\circ} 45^{\prime} 35 \mathrm{E}\end{array}$ & $1100 \mathrm{~m}$ & $\begin{array}{l}\text { A. nordmanniana subsp. } \\
\text { bornmuelleriana forest }\end{array}$ & 22.V.2014 \\
\hline $\mathbf{L 8 3}$ & Sakarya, Akyazı, Kuzuluk Nature Park & $\begin{array}{l}40^{\circ} 37^{\prime} 19^{\prime \prime} \mathrm{N}, \\
30^{\circ} 39^{\prime} 10^{\prime \prime} \mathrm{E}\end{array}$ & $370 \mathrm{~m}$ & $\begin{array}{l}\text { F. orientalis, } \text { C. orientalis, } \\
\text { Quercus sp. forest }\end{array}$ & 18.IV.2013 \\
\hline L84 & $\begin{array}{l}\text { Sakarya, Akyazı, Kuzuluk, on the way to } \\
\text { Yeniköy from the centre }\end{array}$ & $\begin{array}{l}40^{\circ} 38^{\prime} 55^{\prime \prime} \mathrm{N} \\
30^{\circ} 39^{\prime} 12^{\prime \prime} \mathrm{E}\end{array}$ & $260 \mathrm{~m}$ & Quercus sp. forest & 18.IV.2013 \\
\hline $\mathbf{L 8 5}$ & Sakarya, Akyazı, Mansurlar planting area, & $\begin{array}{l}40^{\circ} 34^{\prime} 42^{\prime \prime} \mathrm{N} \\
30^{\circ} 43^{\prime} 24^{\prime \prime} \mathrm{E}\end{array}$ & $280 \mathrm{~m}$ & P. nigra forest & 03.XI.2013 \\
\hline L86 & Sakarya, Akyazı, Özdemirler Plateau & $\begin{array}{l}40^{\circ} 30^{\prime} 12^{\prime \prime} \mathrm{N} \\
30^{\circ} 40^{\prime} 49^{\prime \prime} \mathrm{E}\end{array}$ & $1260 \mathrm{~m}$ & $\begin{array}{l}\text { A. nordmanniana subsp. } \\
\text { bornmuelleriana forest }\end{array}$ & 04.XI.2012 \\
\hline L87 & $\begin{array}{l}\text { Sakarya, Akyazı, Pine planting area on the } \\
\text { Güzlek road }\end{array}$ & $\begin{array}{l}40^{\circ} 39^{\prime} 42^{\prime \prime} \mathrm{N} \\
30^{\circ} 40^{\prime} 07^{\prime \prime} \mathrm{E}\end{array}$ & $225 \mathrm{~m}$ & P. sylvestris forest & 18.IV.2013 \\
\hline L88 & Sakarya, Akyazı, Salihiye & $\begin{array}{l}40^{\circ} 37^{\prime} 09^{\prime \prime} \mathrm{N} \\
30^{\circ} 36^{\prime} 20^{\prime \prime} \mathrm{E}\end{array}$ & $160 \mathrm{~m}$ & Quercus sp. forest & 29.X.2014 \\
\hline L89 & Sakarya, Akyazı, Soğuksu forest building & $\begin{array}{l}40^{\circ} 39^{\prime} 06 " \mathrm{~N}, \\
30^{\circ} 43^{\prime} 37^{\prime \prime} \mathrm{E}\end{array}$ & $930 \mathrm{~m}$ & $\begin{array}{l}\text { A. nordmanniana subsp. } \\
\text { bornmuelleriana forest }\end{array}$ & 02.XI.2013 \\
\hline L90 & $\begin{array}{l}\text { Sakarya, Akyazı, upper part of Kuruçay } \\
\text { Plateau }\end{array}$ & $\begin{array}{l}40^{\circ} 31^{\prime} 25 " \mathrm{~N} \\
30^{\circ} 42^{\prime} 077^{\prime \prime} \mathrm{E}\end{array}$ & $1282 \mathrm{~m}$ & $\begin{array}{l}\text { A. nordmanniana subsp. } \\
\text { bornmuelleriana, F. orientalis } \\
\text { forest }\end{array}$ & 23.V.2014 \\
\hline L91 & $\begin{array}{l}\text { Sakarya, Akyazı, upper part of Özdemirler P } \\
\text { Plateau }\end{array}$ & $\begin{array}{l}40^{\circ} 30^{\prime} 21 " \mathrm{~N} \\
30^{\circ} 40^{\prime} 51^{\prime \prime} \mathrm{E}\end{array}$ & $1300 \mathrm{~m}$ & $\begin{array}{l}\text { A. nordmanniana subsp. } \\
\text { bornmuelleriana forest }\end{array}$ & 23.V.2014 \\
\hline L92 & Sakarya, Akyazı, Yazlık neighbourhood & $\begin{array}{l}40^{\circ} 37^{\prime} 12^{\prime \prime} \mathrm{N} \\
30^{\circ} 36^{\prime} 21^{\prime \prime} \mathrm{E}\end{array}$ & $155 \mathrm{~m}$ & $\begin{array}{l}\text { Quercus sp., C. monogyna, } R \text {. } \\
\text { caesius forest }\end{array}$ & 18.IV.2013 \\
\hline L93 & $\begin{array}{l}\text { Sakarya, Akyazı, Yenikoy, Keremali, side of } \\
\text { the forest building }\end{array}$ & $\begin{array}{l}40^{\circ} 38^{\prime} 47^{\prime \prime} \mathrm{N} \\
30^{\circ} 42^{\prime} 30^{\prime \prime} \mathrm{E}\end{array}$ & $942 \mathrm{~m}$ & $\begin{array}{l}\text { A. nordmanniana subsp. } \\
\text { bornmuelleriana, } P \text {. sylvestris, } F \text {. } \\
\text { orientalis, } R \text {. ponticum forest }\end{array}$ & 03.XI.2012 \\
\hline L94 & $\begin{array}{l}\text { Sakarya, Akyazı, Yeniköy, Keremali, } \\
\text { Kestanelik district }\end{array}$ & $\begin{array}{l}40^{\circ} 38^{\prime} 58^{\prime \prime} \mathrm{N} \\
30^{\circ} 43^{\prime} 33^{\prime \prime} \mathrm{E}\end{array}$ & $882 \mathrm{~m}$ & C. orientalis, $R$. caesius forest & 03.XI.2012 \\
\hline L95 & $\begin{array}{l}\text { Sakarya, Akyazi, Yeniköy, the side of the } \\
\text { Keremali forest building, going to Yeniköy, } \\
\text { with } 500 \mathrm{~m} \text { remaining }\end{array}$ & $\begin{array}{l}40^{\circ} 39^{\prime} 10^{\prime \prime} \mathrm{N} \\
30^{\circ} 43^{\prime} 38^{\prime \prime} \mathrm{E}\end{array}$ & $972 \mathrm{~m}$ & $\begin{array}{l}\text { A. nordmanniana subsp. } \\
\text { bornmuelleriana, } F \text {. orientalis } \\
\text { forest }\end{array}$ & 03.XI.2012 \\
\hline L96 & $\begin{array}{l}\text { Sakarya, Akyazı, Yılanlıkaya turnoff, towards } \\
\text { Avcıçimeni }\end{array}$ & $\begin{array}{l}40^{\circ} 30^{\prime} 59^{\prime \prime} \mathrm{N} \\
30^{\circ} 35^{\prime} 12^{\prime \prime} \mathrm{E}\end{array}$ & $1260 \mathrm{~m}$ & $\begin{array}{l}\text { A. nordmanniana subsp. } \\
\text { bornmuelleriana, F. orientalis, } \\
\text { Quercus sp. forest }\end{array}$ & 01.XI.2013 \\
\hline L97 & $\begin{array}{l}\text { Sakarya, Akyazı, Yörükyeri Vill., between } \\
\text { Civci and Güney neighbourhood, roadside }\end{array}$ & $\begin{array}{l}40^{\circ} 32^{\prime} 49^{\prime \prime} \mathrm{N}, \\
30^{\circ} 45^{\prime} 49^{\prime \prime} \mathrm{E}\end{array}$ & $827 \mathrm{~m}$ & $\begin{array}{l}C \text {. orientalis, } F . \text { orientalis, } \\
\text { Trifolium sp., D. laciniatus forest }\end{array}$ & 04.XI.2012 \\
\hline L98 & Sakarya, Akyazı, Yörükyeri Vill. & $\begin{array}{l}40^{\circ} 31^{\prime} 09^{\prime \prime} \mathrm{N} \\
30^{\circ} 46^{\prime} 17^{\prime \prime} \mathrm{E}\end{array}$ & $1245 \mathrm{~m}$ & F. orientalis forest & 04.XI.2012 \\
\hline L99 & $\begin{array}{l}\text { Sakarya, Akyazı, Zincirlibaba tomb road } \\
\text { separation }\end{array}$ & $\begin{array}{l}40^{\circ} 34^{\prime} 17 " \mathrm{~N} \\
30^{\circ} 37^{\prime} 57^{\prime \prime} \mathrm{E}\end{array}$ & $941 \mathrm{~m}$ & F. orientalis forest & 30.IX.2014 \\
\hline L100 & Sakarya, Akyazı, Zirvedağı & $\begin{array}{l}40^{\circ} 38^{\prime} 54 " \mathrm{~N} \\
30^{\circ} 43^{\prime} 59^{\prime \prime} \mathrm{E}\end{array}$ & $1050 \mathrm{~m}$ & $\begin{array}{l}\text { A. nordmanniana subsp. } \\
\text { bornmuelleriana forest }\end{array}$ & 02.XI.2013 \\
\hline L101 & Sakarya, Geyve, Acielma 2 district & $\begin{array}{l}40^{\circ} 35^{\prime} 47^{\prime \prime} \mathrm{N} \\
30^{\circ} 09^{\prime} 48^{\prime \prime} \mathrm{E}\end{array}$ & $1100 \mathrm{~m}$ & $\begin{array}{l}\text { A. nordmanniana subsp. } \\
\text { bornmuelleriana, } P \text {. nigra, } F \text {. } \\
\text { orientalis, } C \text {. orientalis, } R \text {. } \\
\text { ponticum forest }\end{array}$ & 31.X.2013 \\
\hline
\end{tabular}


Table 1 Continued.

\begin{tabular}{|c|c|c|c|c|c|}
\hline L102 & $\begin{array}{l}\text { Sakarya, Geyve, Acıelma district, Gümüşdere } \\
\text { chiefdom }\end{array}$ & $\begin{array}{l}40^{\circ} 35^{\prime} 56^{\prime \prime} \mathrm{N} \\
30^{\circ} 10^{\prime} 23^{\prime \prime} \mathrm{E}\end{array}$ & $1115 \mathrm{~m}$ & $\begin{array}{l}\text { A. nordmanniana subsp. } \\
\text { Bornmuelleriana, } P \text {. nigra, } F \text {. } \\
\text { orientalis, } C \text {. orientalis, } R \text {. } \\
\text { ponticum forest }\end{array}$ & 30.X.2013 \\
\hline L103 & Sakarya, Geyve, Acielma district & $\begin{array}{l}40^{\circ} 35^{\prime} 49^{\prime \prime} \mathrm{N} \\
30^{\circ} 10^{\prime} 60^{\prime \prime} \mathrm{E}\end{array}$ & $1060 \mathrm{~m}$ & $\begin{array}{l}\text { A. nordmanniana subsp. } \\
\text { bornmuelleriana, } F \text {. orientalis } \\
\text { forest }\end{array}$ & 31.X.2013 \\
\hline L104 & Sakarya, Geyve, Eskiyayla Vill. & $\begin{array}{l}40^{\circ} 32^{\prime} 32^{\prime \prime} \mathrm{N} \\
30^{\circ} 05^{\prime} 12^{\prime \prime} \mathrm{E}\end{array}$ & $935 \mathrm{~m}$ & P. nigra forest & 31.X.2013 \\
\hline L105 & Sakarya, Geyve, Gümüşdere, Kazimiye Vill. & $\begin{array}{l}40^{\circ} 34^{\prime} 00^{\prime \prime} \mathrm{N} \\
30^{\circ} 11^{\prime} 211^{\prime \prime} \mathrm{E}\end{array}$ & $900 \mathrm{~m}$ & P. nigra, Quercus sp. forest & 30.XI.2012 \\
\hline L106 & Sakarya, Geyve, Gümüşdere district & $\begin{array}{l}40^{\circ} 33^{\prime} 55^{\prime \prime} \mathrm{N} \\
30^{\circ} 11^{\prime} 37^{\prime \prime} \mathrm{E}\end{array}$ & $917 \mathrm{~m}$ & P. nigra, Quercus sp. forest & 30.XI.2012 \\
\hline L107 & Sakarya, Geyve, Kaymakam suyu district & $\begin{array}{l}40^{\circ} 35^{\prime} 03^{\prime \prime} \mathrm{N} \\
30^{\circ} 10^{\prime} 40^{\prime \prime} \mathrm{E}\end{array}$ & $970 \mathrm{~m}$ & $\begin{array}{l}\text { A. nordmanniana subsp. } \\
\text { bornmuelleriana, } P \text {. nigra, } F \text {. } \\
\text { orientalis forest }\end{array}$ & 30.XI.2012 \\
\hline L108 & $\begin{array}{l}\text { Sakarya, Geyve, Taraklı, Mahdumlar Vill., } \\
\text { Karagöl Plateau }\end{array}$ & $\begin{array}{l}40^{\circ} 30^{\prime} 17 " \mathrm{~N} \\
30^{\circ} 34^{\prime} 39^{\prime \prime} \mathrm{E}\end{array}$ & $1150 \mathrm{~m}$ & $\begin{array}{l}\text { A. nordmanniana subsp. } \\
\text { bornmuelleriana, } F \text {. orientalis, } C \text {. } \\
\text { orientalis, } B \text {. sempervirens forest }\end{array}$ & 28.V.2014 \\
\hline L109 & $\begin{array}{l}\text { Sakarya, Geyve, Taraklı, Şimşirlikboğazı } \\
\text { district }\end{array}$ & $\begin{array}{l}40^{\circ} 30^{\prime} 55^{\prime \prime} \mathrm{N} \\
30^{\circ} 33^{\prime} 54^{\prime \prime} \mathrm{E}\end{array}$ & $1250 \mathrm{~m}$ & $\begin{array}{l}\text { A. nordmannina subsp. } \\
\text { bornmuelleriana, } B \text {. sempervirens } \\
\text { forest }\end{array}$ & 28.V.2014 \\
\hline L110 & $\begin{array}{l}\text { Sakarya, Geyve, Taraklı, upper part of } \\
\text { Dişdedeler Plateau }\end{array}$ & $\begin{array}{l}40^{\circ} 31^{\prime} 05^{\prime \prime} \mathrm{N} \\
30^{\circ} 32^{\prime} 38^{\prime \prime} \mathrm{E}\end{array}$ & $1315 \mathrm{~m}$ & $\begin{array}{l}\text { A. nordmannina subsp. } \\
\text { bornmuelleriana, B. sempervirens } \\
\text { forest }\end{array}$ & 28.V.2014 \\
\hline L111 & Sakarya, Göktepe, the place of Pala district & $\begin{array}{l}40^{\circ} 34^{\prime} 53 " \mathrm{~N} \\
30^{\circ} 32^{\prime} 34^{\prime \prime} \mathrm{E}\end{array}$ & $926 \mathrm{~m}$ & $\begin{array}{l}C . \text { sativa, } F . \text { orientalis, } C \text {. } \\
\text { orientalis, } R \text {. ponticum, } R \text {. sanctus } \\
\text { forest }\end{array}$ & 02.XI.2012 \\
\hline L112 & Sakarya, Karapürçek district & $\begin{array}{l}40^{\circ} 34^{\prime} 55^{\prime \prime} \mathrm{N} \\
30^{\circ} 29^{\prime} 56^{\prime \prime} \mathrm{E}\end{array}$ & $1160 \mathrm{~m}$ & $\begin{array}{l}\text { A. nordmanniana subsp. } \\
\text { bornmuelleriana, F. orientalis } \\
\text { forest }\end{array}$ & 28.X.2014 \\
\hline L113 & Sakarya, Karapürçek, Uludere district & $\begin{array}{l}40^{\circ} 36^{\prime} 07 " \mathrm{~N} \\
30^{\circ} 30^{\prime} 36^{\prime \prime} \mathrm{E}\end{array}$ & $570 \mathrm{~m}$ & $\begin{array}{l}\text { F. orientalis, } C \text {. sativa, } C \text {. } \\
\text { orientalis forest }\end{array}$ & 28.X.2014 \\
\hline L114 & Sakarya, Pamukova, Atalanı district & $\begin{array}{l}40^{\circ} 33^{\prime} 08^{\prime \prime} \mathrm{N} \\
30^{\circ} 06^{\prime} 04^{\prime \prime} \mathrm{E}\end{array}$ & $870 \mathrm{~m}$ & $\begin{array}{l}\text { A. nordmanniana subsp. } \\
\text { bornmuelleriana, } P \text {. nigra, } \\
\text { Quercus sp. forest }\end{array}$ & 29.XI.2012 \\
\hline L115 & Sakarya, Pamukova, Bakacak Vill. & $\begin{array}{l}40^{\circ} 33^{\prime} 05^{\prime \prime} \mathrm{N} \\
30^{\circ} 06^{\prime} 01^{\prime \prime} \mathrm{E}\end{array}$ & $910 \mathrm{~m}$ & P. nigra, Quercus sp. forest & 31.X.2013 \\
\hline L116 & $\begin{array}{l}\text { Sakarya, Pamukova, Katırözü, forest } \\
\text { warehouse }\end{array}$ & $\begin{array}{l}40^{\circ} 31^{\prime} 58 " \mathrm{~N} \\
30^{\circ} 03^{\prime} 51^{\prime \prime} \mathrm{E}\end{array}$ & $800 \mathrm{~m}$ & $\begin{array}{l}\text { A. nordmanniana subsp. } \\
\text { bornmuelleriana, } P \text {. nigra, } F \text {. } \\
\text { orientalis forest }\end{array}$ & 29.XI.2012 \\
\hline L117 & $\begin{array}{l}\text { Sakarya, Pamukova, Şehitlik district, forest } \\
\text { camp }\end{array}$ & $\begin{array}{l}40^{\circ} 31^{\prime} 00^{\prime \prime} \mathrm{N} \\
29^{\circ} 59^{\prime} 30^{\prime \prime} \mathrm{E}\end{array}$ & $1105 \mathrm{~m}$ & $\begin{array}{l}\text { A. nordmanniana subsp. } \\
\text { bornmuelleriana, F. orientalis } \\
\text { forest }\end{array}$ & 29.IX.2014 \\
\hline L118 & $\begin{array}{l}\text { Sakarya, Pamukova, Soğucak way, under the } \\
\text { transmitter }\end{array}$ & $\begin{array}{l}40^{\circ} 33^{\prime} 18^{\prime \prime} \mathrm{N} \\
30^{\circ} 11^{\prime} 02^{\prime \prime} \mathrm{E}\end{array}$ & $1000 \mathrm{~m}$ & $\begin{array}{l}\text { F. orientalis, } P \text {. nigra, Quercus } \\
\text { sp. forest }\end{array}$ & 27.X.2014 \\
\hline L119 & $\begin{array}{l}\text { Sakarya, Pamukova, the upper part of Ahiler } \\
\text { Vill. }\end{array}$ & $\begin{array}{l}40^{\circ} 29^{\prime} 38^{\prime \prime} \mathrm{N} \\
30^{\circ} 00^{\prime} 52^{\prime \prime} \mathrm{E}\end{array}$ & $631 \mathrm{~m}$ & $\begin{array}{l}\text { P. nigra, P. brutia, } R \text {. sanctus } \\
\text { forest }\end{array}$ & 29.XI.2012 \\
\hline L120 & $\begin{array}{l}\text { Sakarya, Pamukova, upper part of Kazımiye } \\
\text { Vill. }\end{array}$ & $\begin{array}{l}40^{\circ} 33^{\prime} 53 " \mathrm{~N} \\
30^{\circ} 11^{\prime} 33^{\prime \prime} \mathrm{E}\end{array}$ & $930 \mathrm{~m}$ & P. nigra, Quercus sp. forest & 27.X.2014 \\
\hline L121 & Sakarya, Sapanca, Çakılocağı district & $\begin{array}{l}40^{\circ} 37^{\prime} 10^{\prime \prime} \mathrm{N} \\
30^{\circ} 14^{\prime} 25^{\prime \prime} \mathrm{E}\end{array}$ & $970 \mathrm{~m}$ & $\begin{array}{l}\text { A. nordmanniana subsp. } \\
\text { bornmuelleriana, Salix sp., } C \text {. } \\
\text { sativa, C. orientalis forest }\end{array}$ & 26.XI.2012 \\
\hline $\mathbf{L 1 2 2}$ & $\begin{array}{l}\text { Sakarya, Sapanca, Geyve entrance from } \\
\text { Soğucak Plateau }\end{array}$ & $\begin{array}{l}40^{\circ} 36 ' 20^{\prime \prime} \mathrm{N} \\
30^{\circ} 11^{\prime} 34^{\prime \prime} \mathrm{E}\end{array}$ & $1115 \mathrm{~m}$ & F. orientalis forest & 30.X.2013 \\
\hline L123 & Sakarya, Sapanca, Memnuniye Vill. & $\begin{array}{l}40^{\circ} 38^{\prime} 10^{\prime \prime} \mathrm{N} \\
30^{\circ} 15^{\prime} 09^{\prime \prime} \mathrm{E}\end{array}$ & $850 \mathrm{~m}$ & $\begin{array}{l}\text { A. nordmanniana subsp. } \\
\text { bornmuelleriana, Salix } \mathrm{sp} ., \text { C. } \\
\text { sativa, C. orientalis forest }\end{array}$ & 26.XI.2012 \\
\hline L124 & Sakarya, Sapanca, Soğucak Plateau entrance & $\begin{array}{l}40^{\circ} 36^{\prime} 55^{\prime \prime} \mathrm{N} \\
30^{\circ} 10^{\prime} 52^{\prime \prime} \mathrm{E}\end{array}$ & $1200 \mathrm{~m}$ & $\begin{array}{l}\text { A. nordmanniana subsp. } \\
\text { bornmuelleriana, } P \text {. nigra, } P \text {. } \\
\text { sylvestris forest }\end{array}$ & 27.IX.2014 \\
\hline L125 & $\begin{array}{l}\text { Sakarya, Sapanca, Soğucak Plateau road, } \\
\text { Chestnut area }\end{array}$ & $\begin{array}{l}40^{\circ} 39^{\prime} 34^{\prime \prime} \mathrm{N} \\
30^{\circ} 13^{\prime} 57^{\prime \prime} \mathrm{E}\end{array}$ & $477 \mathrm{~m}$ & $\begin{array}{l}\text { C. sativa, } F \text {. orientalis, } C \text {. } \\
\text { orientalis, } P \text {. nigra forest }\end{array}$ & 27.IX.2014 \\
\hline
\end{tabular}


Table 1 Continued.

\begin{tabular}{|c|c|c|c|c|c|}
\hline L126 & Sakarya, Sapanca, Soğucak Plateau & $\begin{array}{l}40^{\circ} 34^{\prime} 35 " \mathrm{~N}, \\
30^{\circ} 09^{\prime} 59^{\prime \prime} \mathrm{E}\end{array}$ & $1100 \mathrm{~m}$ & $\begin{array}{l}\text { A. nordmanniana subsp. } \\
\text { bornmuelleriana, F. orientalis } \\
\text { forest }\end{array}$ & 14.VI.2012 \\
\hline L127 & $\begin{array}{l}\text { Sakarya, Sapanca, Soğucak Plateau, on the } \\
\text { Geyve dam road }\end{array}$ & $\begin{array}{l}40^{\circ} 36^{\prime} 20^{\prime \prime} \mathrm{N} \\
30^{\circ} 11^{\prime} 41 " \mathrm{E}\end{array}$ & $1190 \mathrm{~m}$ & C. orientalis forest & 25.V.2014. \\
\hline L128 & $\begin{array}{l}\text { Sakarya, Sapanca, upper part of Memnuniye } \\
\text { Vill. }\end{array}$ & $\begin{array}{l}40^{\circ} 38^{\prime} 49^{\prime \prime} \mathrm{N} \\
30^{\circ} 15^{\prime} 17^{\prime \prime} \mathrm{E}\end{array}$ & $760 \mathrm{~m}$ & $\begin{array}{l}\text { Quercus sp., F. orientalis, } C . \\
\text { orientalis forest }\end{array}$ & 15.IV.2013 \\
\hline L129 & Sakarya, Suadiye, Kuzuyayla district & $\begin{array}{l}40^{\circ} 38^{\prime} 52^{\prime \prime} \mathrm{N} \\
30^{\circ} 07^{\prime} 02^{\prime \prime} \mathrm{E}\end{array}$ & $1400 \mathrm{~m}$ & $\begin{array}{l}\text { F. orientalis, } C \text {. sativa, } C \text {. } \\
\text { orientalis forest }\end{array}$ & 16.IV.2013 \\
\hline L130 & $\begin{array}{l}\text { Sakarya, Suadiye, Taşkonak villas, upward of } \\
\text { Motali }\end{array}$ & $\begin{array}{l}40^{\circ} 41^{\prime} 24 " \mathrm{~N} \\
30^{\circ} 08^{\prime} 00^{\prime \prime} \mathrm{E}\end{array}$ & $280 \mathrm{~m}$ & P. nigra, Quercus sp. forest & 16.IV.2013 \\
\hline L131 & Yalova, Armutlu, partition no 149 & $\begin{array}{l}40^{\circ} 32^{\prime} 01 " \mathrm{~N} \\
28^{\circ} 59^{\prime} 97^{\prime \prime} \mathrm{E}\end{array}$ & $760 \mathrm{~m}$ & P. nigra forest & 06.VI.2013 \\
\hline L132 & Yalova, Armutlu to Karapinar, partition no 64 & $\begin{array}{l}40^{\circ} 33^{\prime} 21 " \mathrm{~N} \\
28^{\circ} 57^{\prime} 61^{\prime \prime} \mathrm{E}\end{array}$ & $587 \mathrm{~m}$ & F. orientalis forest & 06.VI.2013 \\
\hline L133 & Yalova, Armutlu, Delmece Plateau & $\begin{array}{l}40^{\circ} 32^{\prime} 44 " \mathrm{~N}, \\
29^{\circ} 00^{\prime} 15^{\prime \prime} \mathrm{E}\end{array}$ & $765 \mathrm{~m}$ & F. orientalis, C. orientalis forest & 06.VI.2013 \\
\hline L134 & Yalova, Armutlu, Mecidiye Vill. & $\begin{array}{l}40^{\circ} 30^{\prime} 42^{\prime \prime} \mathrm{N} \\
28^{\circ} 54^{\prime} 42^{\prime \prime} \mathrm{E}\end{array}$ & $495 \mathrm{~m}$ & P. maritima forest & 10.X.2014 \\
\hline L135 & Yalova, Armutlu, partition no 151 & $\begin{array}{l}40^{\circ} 32^{\prime} 37^{\prime \prime} \mathrm{N}, \\
29^{\circ} 00^{\prime} 16^{\prime \prime} \mathrm{E}\end{array}$ & $780 \mathrm{~m}$ & $F$. orientalis, $P$. nigra forest & 06.VI.2013 \\
\hline L136 & Yalova, Beşpınar Plateau & $\begin{array}{l}40^{\circ} 32^{\prime} 03^{\prime \prime} \mathrm{N} \\
29^{\circ} 13^{\prime} 18^{\prime \prime} \mathrm{E}\end{array}$ & $720 \mathrm{~m}$ & $\begin{array}{l}\text { F. orientalis, C. orientalis, Tilia } \\
\text { sp. forest }\end{array}$ & 07.VI.2013 \\
\hline L137 & Yalova, Çanakpınar Plateau & $\begin{array}{l}40^{\circ} 32^{\prime} 40^{\prime \prime} \mathrm{N} \\
29^{\circ} 11^{\prime} 34^{\prime \prime} \mathrm{E}\end{array}$ & $700 \mathrm{~m}$ & F. orientalis forest & 07.VI.2013 \\
\hline L138 & Yalova, Çınarcık, Delmece Plateau entrance & $\begin{array}{l}40^{\circ} 32^{\prime} 47 " \mathrm{~N}, \\
29^{\circ} 00^{\prime} 20^{\prime \prime} \mathrm{E}\end{array}$ & $800 \mathrm{~m}$ & $F$. orientalis, $P$. sylvestris, forest & 03.VI.2014 \\
\hline L139 & Yalova, Çınarcık, Delmece Plateau & $\begin{array}{l}40^{\circ} 32^{\prime} 44 " \mathrm{~N} \\
29^{\circ} 00^{\prime} 15^{\prime \prime} \mathrm{E}\end{array}$ & $765 \mathrm{~m}$ & F. orientalis, C. orientalis forest & 03.VI.2014 \\
\hline L140 & $\begin{array}{l}\text { Yalova, Çınarcık, Karlık Plateau, partition no } \\
197,242\end{array}$ & $\begin{array}{l}40^{\circ} 34^{\prime} 86^{\prime \prime} \mathrm{N} \\
28^{\circ} 59^{\prime} 38^{\prime \prime} \mathrm{E}\end{array}$ & $840 \mathrm{~m}$ & F. orientalis, young forest & 06.VI.2013 \\
\hline L141 & $\begin{array}{l}\text { Yalova, Çınarcık, Teşvikiye, Dipsizgöller } \\
\text { district }\end{array}$ & $\begin{array}{l}40^{\circ} 37^{\prime} 25 " \mathrm{~N} \\
29^{\circ} 05^{\prime} 21^{\prime \prime} \mathrm{E}\end{array}$ & $595 \mathrm{~m}$ & F. orientalis, Quercus sp. forest & 03.VI.2014 \\
\hline L142 & $\begin{array}{l}\text { Yalova, Çınarcık, Teşvikiye Vill., partition no } \\
15\end{array}$ & $\begin{array}{l}40^{\circ} 35^{\prime} 25^{\prime \prime} \mathrm{N}, \\
29^{\circ} 00^{\prime} 21^{\prime \prime} \mathrm{E}\end{array}$ & $600 \mathrm{~m}$ & P. maritima forest & 11.XI.2014 \\
\hline L143 & $\begin{array}{l}\text { Yalova, Çınarcık, Teşvikiye Vill., partition no } \\
161\end{array}$ & $\begin{array}{l}40^{\circ} 36^{\prime} 39 " \mathrm{~N}, \\
26^{\circ} 05^{\prime} 41^{\prime \prime} \mathrm{E}\end{array}$ & $500 \mathrm{~m}$ & $F$. orientalis, $C$. sativa forest & 11.XI.2014 \\
\hline L144 & $\begin{array}{l}\text { Yalova, Çınarcık, Teşvikiye Vill., partition no } \\
200\end{array}$ & $\begin{array}{l}40^{\circ} 37^{\prime} 30^{\prime \prime} \mathrm{N} \\
29^{\circ} 08^{\prime} 42^{\prime \prime} \mathrm{E}\end{array}$ & $200 \mathrm{~m}$ & P. maritima forest & 11.XI.2014 \\
\hline L145 & Yalova, Çınarcık, Urban forest & $\begin{array}{l}40^{\circ} 35^{\prime} 46 " \mathrm{~N} \\
29^{\circ} 02^{\prime} 29^{\prime \prime} \mathrm{E}\end{array}$ & $475 \mathrm{~m}$ & F. orientalis, Quercus sp. forest & 03.VI.2014 \\
\hline L146 & Yalova, Haydariye Vill., partition no 35 & $\begin{array}{l}40^{\circ} 33^{\prime} 28^{\prime \prime} \mathrm{N} \\
29^{\circ} 06^{\prime} 27^{\prime \prime} \mathrm{E}\end{array}$ & $550 \mathrm{~m}$ & F. orientalis forest & 26.X.2013 \\
\hline L147 & $\begin{array}{l}\text { Yalova, Termal, on the way of Haydariye } \\
\text { Vill. }\end{array}$ & $\begin{array}{l}40^{\circ} 34^{\prime} 47 " \mathrm{~N} \\
29^{\circ} 09^{\prime} 08^{\prime \prime} \mathrm{E}\end{array}$ & $210 \mathrm{~m}$ & F. orientalis, $C$. sativa forest & 03.VI.2014 \\
\hline L148 & Yalova, Termal, Suyolu district & $\begin{array}{l}40^{\circ} 34^{\prime} 54 " \mathrm{~N}, \\
29^{\circ} 10^{\prime} 40^{\prime \prime} \mathrm{E}\end{array}$ & $200 \mathrm{~m}$ & $F$. orientalis, $C$. sativa forest & 03.VI.2014 \\
\hline
\end{tabular}

Abbreviations; (E): edible, (F): used as food, (I): inedible, (M): used for medical purposes, (P): poisonous, (U): unknown, (?): suspicious, (L): locality.

\section{Results}

\section{Division ASCOMYCOTA \\ Order Coronophorales \\ Family Bertiaceae}

Bertia moriformis (Tode) De Not.: (I), L127, saprobe on herbaceous and woody tissue.

\section{Order Helotiales}

Family Helotiaceae

Hymenoscyphus calyculus (Fr.) W. Phillips: (I), L70, L86, saprobe.
Hymenoscyphus serotinus (Pers.) W. Phillips: (I), L60, L67, L68, L103, L122, L146, saprobe.

\section{Family Lachnaceae}

Dasyscyphella nivea (R. Hedw.) Raitv.: (I), L46, saprobe.

Lachnellula calyciformis (Batsch) Dharne: (I), L79, saprobe.

Lachnellula occidentalis (G.G. Hahn \& Ayers) Dharne: (I), L95, saprobe.

Lachnellula subtilissima (Cooke) Dennis: (I), L126, saprobe. 
Lachnum virgineum (Batsch) P. Karst.: (I), L11, L20, L36, L83, L124, saprobe.

\section{Family Pezizellaceae}

Calycina citrina (Hedw.) Gray: (I), L24, L41, L588, L63, L65, L70, L78, L95, L102, L119, L122, saprobe.

Calycina parilis (P. Karst.) Kuntze: (I), L63, saprobe. Family Rutstroemiaceae

Rutstroemia firma (Pers.) P. Karst.: (I), L63, saprobe. Family Sclerotiniaceae

Ciboria amentacea (Balb.) Fuckel: (I), L49, saprobe.

Order Hypocreales

Family Cordycipitaceae

Ophiocordyceps gracilis (Grev.) G.H. Sung, J.M. Sung, Hywel-Jones \& Spatafora: (M), L57, on caterpillar, entomopathogenic.

Family Nectriaceae

Nectria cinnabarina (Tode) Fr.: (I), L126, saprobe.

Order Leotiacae

Family Leotiaceae

Leotia lubrica (Scop.) Pers.: (I), L3, L65, L126, L146, saprobe.

Order Pezizales

Family Caloscyphaceae

Caloscypha fulgens (Pers.) Boud.: (I), L66, parasite on the seeds of conifers.

\section{Family Helvellaceae}

Dissingia leucomelaena (Pers.) K. Hansen \& X.H. Wang: (E, or ?), L75, mycorrhizal.

Helvella acetabulum (L.) Quél.: (E, or ?), L73, mycorrhizal.

Helvella atra J. König: (I), L144, mycorrhizal.

Helvella crispa (Scop.) Fr.: (E, or ?), L93, L112, L125, mycorrhizal.

Helvella elastica Bull.: (E), L3, L41, L112, L147, mycorrhizal.

Helvella fibrosa (Wallr.) Korf: (I), L79, mycorrhizal.

Helvella lacunosa Afzel.: (E, or ?), L5, L58, L71, L83, mycorrhizal.

Helvella leucophaea (Battarra) Pers.: (I), L85, mycorrhizal.

\section{Family Morchellaceae}

Morchella esculenta (L.) Pers.: (E), L130, mycorrhizal.

\section{Family Pezizaceae}

Legaliana badia (Pers.) Van Vooren: (I), L3, L133, mycorrhizal.
Pachyella celtica (Boud.) Häffne: (I), L47, L79, saprobe.

Paragalactinia succosa (Berk.) Van Vooren: (I), L49, saprobe.

Peziza arvernensis Roze \& Boud.: (I), L70, L75, saprobe.

Peziza depressa Pers.: (I), L112, saprobe.

Peziza micropus Pers.: (I), L83, saprobe.

Sarcosphaera coronaria (Jacq.) J. Schröt.: (E, or ?), L73, L74, saprobe.

Family Pyronemataceae

Aleuria aurantia (Pers.) Fuckel: (I), L38, L60, L70, L101, saprobe.

Aleuria splendens (Quél.) Gillet: (I), L64, L111, saprobe.

Geopora sumneriana (Cooke ex W. Phillips) M. Torre: (I), L766, saprobe.

Humaria hemisphaerica (F.H. Wigg.) Fuckel: (I), L52, L70, saprobe.

Otidea alutacea (Pers.) Massee: (I), L103, L10, L126, saprobe.

Tarzetta catinus (Holmsk.) Korf \& J.K. Rogers: (I), L58, L68, L70, L71, L73, saprobe.

Tarzetta cupularis (L.) Lambotte: (I), L70, saprobe.

Family Sarcoscyphaceae

Sarcoscypha coccinea (Gray) Boud.: (I), L5, L143, saprobe.

\section{Order Xylariales \\ Family Diatrypaceae}

Diatrype disciformis (Hoffm.) Fr.: (I), L5, L11, L16, L17, L21, L29, L30, L35, L58, L59, L65, L66, L67, L78, L83, L98, L103, L107, L126, L127, L132, L133, L135, L136, L137, L139, saprobe.

Diatrype stigma (Hoffm.) Fr.: (I), L63, L68, L137, saprobe.

Eutypa acharii Tul. \& C. Tul.: (I), L102, saprobe.

\section{Family Graphostromataceae}

Biscogniauxia nummularia (Bull.) Kuntze: (I), L62, endophytic.

\section{Family Hypoxylaceae}

Daldinia concentrica (Bolton) Ces. \& De Not.: (M), L60, saprobe.

Hypoxylon fragiforme (Pers.) J. Kickx f.: (I), L36, L52, L81, L88, L128, L136, saprobe.

Hypoxylon macrosporum P. Karst.: (I), L78, saprobe.

Hypoxylon rutilum Tul. \& C. Tul.: (I), L6, saprobe.

Jackrogersella cohaerens (Pers.) L. Wendt, Kuhnert \& M. Stadler: (I), L94, saprobe. 
Jackrogersella multiformis (Fr.) L. Wendt, Kuhnert \& M. Stadler: (I), L46, L63, L78, L127, L126, L127, L132, L137, saprobe.

\section{Family Melogrammataceae}

Melogramma campylosporum Fr.: (I), L81, saprobe.

Melogramma spiniferum (Wallr.) De Not.: (I), L58, saprobe.

\section{Family Xylariaceae}

Kretzschmaria deusta (Hoffm.) P.M.D. Martin: (I), L126, saprobe.

Rosellinia mammiformis (Pers.) Ces. \& De Not.: (I), L60, L83, L88, saprobe.

Xylaria hypoxylon (L.) Grev.: (I), L111, saprobe.

Xylaria longipes Nitschke: (I), L125, saprobe.

Xylaria polymorpha (Pers.) Grev.: (M), L46, L56, L57, L63, L70, L86, L126, saprobe.

\section{Division BASIDIOMYCOTA Order Agaricales Family Agaricaceae}

Agaricus arvensis Schaeff.: (E), L108, saprobe.

Agaricus bisporus (J.E. Lange) Imbach: (E), L70, saprobe.

Agaricus bresadolanus Bohus: (P), L90, saprobe.

Agaricus campestris L.: (F), L72, saprobe.

Agaricus comtulus Fr.: (E), L7, saprobe.

Agaricus cupreobrunneus (Jul.Schäff. \& Steer) Pilát: (E), L115, saprobe.

Agaricus essettei Bon: (E), L18, L65, saprobe.

Agaricus langei (F.H. Møller) F.H. Møller: (E), L65, saprobe.

Agaricus moelleri Wasser: (P), L52, saprobe.

Agaricus pampeanus Speg.: (E), L18, L126, saprobe.

Agaricus sylvicola (Vittad.) Peck: (E), L30, saprobe.

Agaricus xanthoderma Genev.: (P), L65, L69, L126, saprobe.

Chlorophyllum brunneum (Farl. \& Burt) Vellinga: (E, or ?), L78, saprobe.

Chlorophyllum rhacodes (Vittad.) Vellinga: (E), L104, saprobe.

Coprinus comatus (O.F. Müll.) Pers.: (F), L86, L97, saprobe.

Crucibulum laeve (Huds.) Kambly: (I), L41, L70, L79, L93, L119, saprobe.

Cyathus olla (Batsch) Pers.: (I), L99, saprobe.

Cystoderma amianthinum (Scop.) Fayod: (I), L65, L118, saprobe.

Cystoderma carcharias (Pers.) Fayod: (I), L65, saprobe.
Cystodermella granulosa (Batsch) Harmaja: (I), L81, L120, saprobe.

Lepiota clypeolaria (Bull.) P. Kumm.: (P), L09, L108, L120, saprobe.

Lepiota cristata (Bolton) P. Kumm.: (P), L15, L57, saprobe.

Lepiota ignivolvata Bousset \& Joss. ex Joss: (P), L18, L30, saprobe.

Lepiota kuehneri Huijsman: (P), L85, saprobe.

Lepiota oreadiformis Velen.: (P), L49, L137, saprobe.

Leucoagaricus leucothites (Vittad.) Wasser: (E), L48, L51, saprobe.

Macrolepiota excoriata (Schaeff.) Wasser: (E), L70, L1012, L126, saprobe.

Macrolepiota heimii (Locq.) Bon: (E), L51, saprobe.

Macrolepiota mastoidea (Fr.) Singer: (E), L10, L18, L60, L65, L66, L86, L103, L116, L126, saprobe.

Macrolepiota procera (Scop.) Singer: (F), L18, L21, L23, L30, L34, L45, L48, L54, L57, L64, L65, L66, L70, L84, L85, L106, L107, L114, L120, L126, L139, saprobe.

Mycenastrum corium (Guers.) Desv.: (E), L15, saprobe. Family Amanitaceae

Amanita battarrae (Boud.) Bon: (U), L39, mycorrhizal.

Amanita caesarea (Scop.) Pers.: (F), L10, L18, L112, mycorrhizal.

Amanita citrina Pers.: (P), L18, L30, L44, L478, L63, L65, L66, L102, L103, L107, L117, L120, L126, L139, mycorrhizal.

Amanita echinocephala (Vittad.) Quél.: (I), L26, L133, mycorrhizal.

Amanita excelsa (Fr.) Bertill.: (E), L17, L20, L34, L64, L100, L124, L137, L141, L146, mycorrhizal.

Amanita franchetii (Boud.) Fayod: (I), L113, L138, mycorrhizal.

Amanita gemmata (Fr.) Bertill.: (P), L3, L8, L33, L35, L43, L44, L46, L58, L62, L65, L66, L69, L77, L81, L82, L108, L124, L126, L127, L132, L136, L138, L139 L140, L141, L143, L145, mycorrhizal.

Amanita mairei Foley: (I), L5, L10, L58, L88, L30, mycorrhizal.

Amanita muscaria (L.) Lam.: (P), L18, L55, L64, L65, L70, L79, L89, L95, L96, L100, L102, L107, L124, L126, mycorrhizal.

Amanita nivalis Grev.: (U), L8, mycorrhizal.

Amanita pantherina (DC.) Krombh.: (P), L8, L10, L52, L60, L63, L93, L95, L118, L139, mycorrhizal.

Amanita phalloides (Vaill. ex Fr.) Link: (P), L17, L34, L38, L81, L125, L135, L139, L140, L141, mycorrhizal. 
Amanita rubescens Pers.: (E), L4, L8, L11, L17, L18, L20, L26, L32, L33, L34, L35, L36, L40, L60, L6, L64, L65, L66, L67, L95, L97, L124, L133, L135, L136, L137, L138, L139, L140, L141, L147, L148, mycorrhizal.

Amanita solitaria (Bull.) Mérat: (P), L4, mycorrhizal.

Amanita submembranacea (Bon) Gröger: (U), L4, L9, L11, L36, mycorrhizal.

Amanita subnudipes (Romagn.) Tulloss: (E), (New record for Turkey)

Pileus 30-80 mm wide, conic at first, then convex, mat, with a striate margin, pale pure orange or with a more yellow tint (Fig. 2a). Flesh white, orange-ocherish under the cuticle, thin, almost odourless, taste mild. Lamellae free, subcrowded, and whitish, short lamellae are infrequent. Stipe 110-140 × 12-20 mm, cylindrical, white, or very pale, fragile, exannulate, hollow. The sac-like volva is white, membranous, thin, tall, and persistent. Spores (5-)7.5-10(-13) × (6-)9-12(-18) $\mu \mathrm{m}$, subglobose to broadly ellipsoid (rarely globose or ellipsoid or narrower) and inamyloid (Fig. 2b). Basidia 10-12 × 50-55 $\mu \mathrm{m}$, cylindrical to subclavate, 4-spored (Fig. 2c).

Distribution: L11, under Quercus sp., mycorrhizal.

Remarks: While this species was previously described as Amanita crocea var. subnudipes Romagn., it was raised to the species level by Tullos (2000). It is easily separated from Amanita crocea with its pure orange or with a more yellow tint pileus, white or very pale and lacking contrasting fibrillose decoration stipe.

Amanita vaginata (Bull.) Lam.: (E), L4, L5, L9, L11, L18, L21, L27, L34, L37, L38, L40, L67, L68, L81, L108, L127, L137, L138, L146, L147, L148, mycorrhizal.

Amanita verna (Bull.) Lam.: (P), L35, L141, L142, mycorrhizal.

Amanita virosa Bertill.: (P), L47, L137, L140, mycorrhizal.

Zhuliangomyces illinitus (Fr.) Redhead: (E), L40, saprobe.

\section{Family Cortinariaceae}

Cortinarius aureofulvus M.M. Moser: (I), L108, mycorrhizal.

Cortinarius elegantissimus Rob. Henry: (I), L40, mycorrhizal.

Cortinarius humicola (Quél.) Maire: (P), L66, L144, mycorrhizal.

Cortinarius melanotus Kalchbr.: (I), L2, mycorrhizal.

Cortinarius orellanus Fr.: (P), L2, mycorrhizal.

Family Crepidotaceae

Crepidotus luteolus Sacc.: (I), L85, L91, mycorrhizal.

Crepidotus variabilis (Pers.) P. Kumm.: (I), L7, L85, saprobe.
Family Entolomataceae

Clitopilus prunulus (Scop.) P. Kumm.: (E), L87, L25, saprobe.

Entoloma lividoalbum (Kühner \& Romagn.) Kubička: (I), L40, saprobe.

Entoloma rhodopolium (Fr.) P. Kumm.: (P), L6, L108, saprobe.

Entoloma sinuatum (Bull. ex Pers.) P. Kumm.: (P), L11, saprobe.

\section{Family Fistulinaceae}

Fistulina hepatica (Schaeff.) With.: (E), L50, L84, saprobe, or weakly parasite, causes a brown rot.

\section{Family Hydnangiaceae}

Laccaria amethystina Cooke: (E), L4, L48, L53, L66, L79, L80, L94, L108, L109, L118, L125, L127, L145, L147, mycorrhizal.

Laccaria laccata (Scop.) Cooke: (E), L4, L42, L47, L48, L53, L62, L64, L66, L94, L96, L109, L108, L112, L127, L22, mycorrhizal.

Laccaria proxima (Boud.) Pat.: (E), L95, mycorrhizal. Family Hygrophoraceae

Ampulloclitocybe clavipes (Pers.) Redhead, Lutzoni, Moncalvo \& Vilgalys: (E), L84, L118, saprobe.

Cantharellula umbonata (J.F. Gmel.) Singer: (E), L66, mycorrhizal.

Chrysomphalina chrysophylla (Fr.) Clémençon: (U), L53, L66, saprobe.

Hygrocybe conica (Schaeff.) P. Kumm.: (I), L89, L119, L127, saprobe.

Hygrocybe obrussea (Fr.) Wunsche: (E), (New record for Turkey)

Pileus 15-30(70) mm across, campanulate, obtusely conic at first, later conic-campanulate to plane, often with an obtuse umbo, surface somewhat butyraceous when moist, satiny, dull when dry, orange to yellow-orange or reddish-orange when young, later fading to grey or oliveyellow or olive-brownish, margin acute, somewhat cleft, barely striate (Fig. 3a). Flesh lemon to orange-yellow coloured, thin, odour like Lactarius quietus, taste mild, somewhat unpleasant. Lamellae broad, yellow to yelloworange, broadly adnexed and sometimes decurrent as a tooth, edges yellowish, smooth.

Stipe 4-10 × 25-60 mm, cylindric, somewhat flexuous, at times somewhat compressed, surface smooth, longitudinally fibrillose, dry, with translucent crossbands, yellow-orange to orange, base sometimes whitish, hollow, elastic. Spores 3.5-5 × 7-9.5 $\mu \mathrm{m}$, ellipticcylindric, usually constricted, smooth, hyaline, with drops (Fig. 3b). Basidia 40-50 × 7-8.5 $\mu \mathrm{m}$, clavate, with 4sterigmata and basal clamp (Fig. 3b).

Distribution: L40, under Quercus sp., saprobe. 


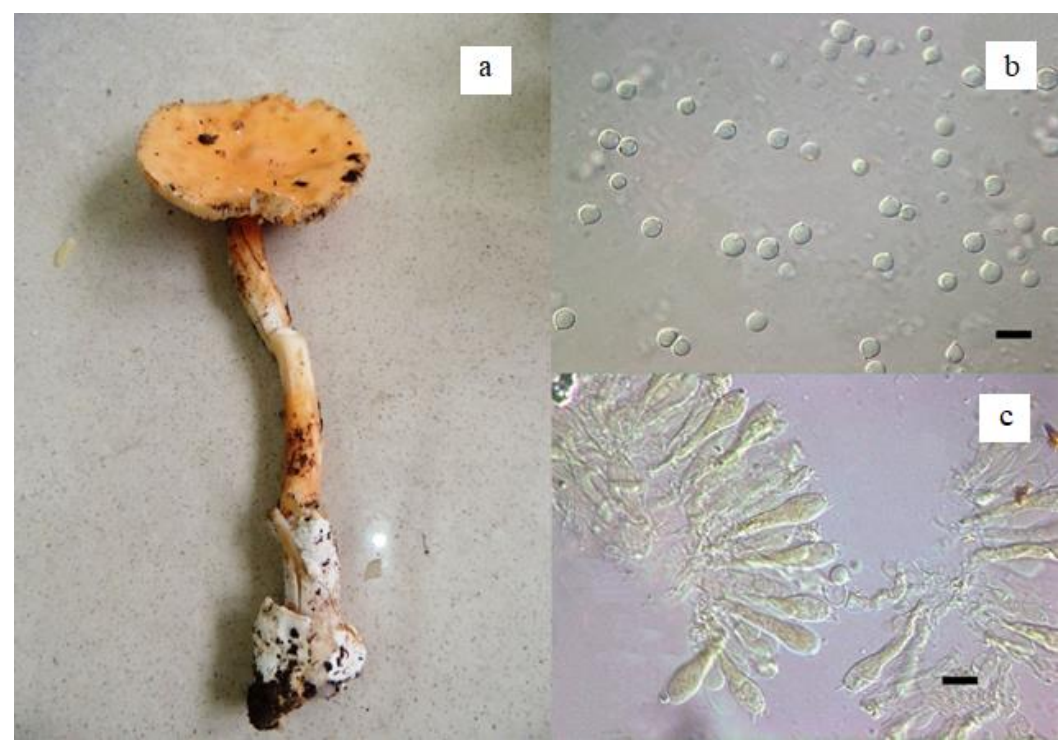

Fig. 2. Amanita subnudipes. a) Macroscopic view, b) basidiospores, c) basidia. Scales $15 \mu \mathrm{m}$.

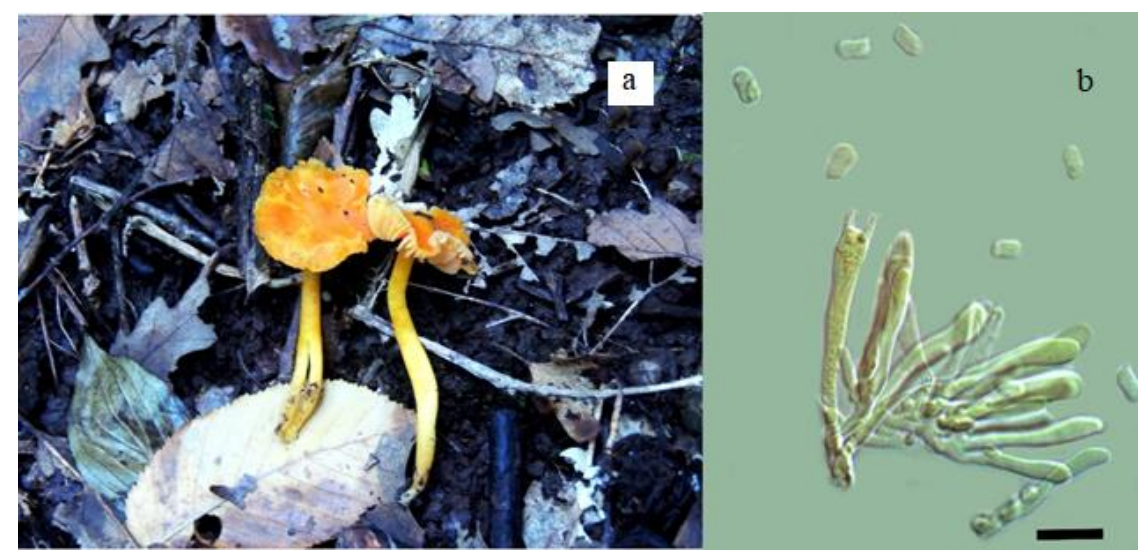

Fig. 3. Hygrocybe obrussea. a) Macroscopic view, b) basidiospores and basidia, Scale $15 \mu \mathrm{m}$.

Remarks: In addition to the dry stipe, the characteristic features of this species are the +/broadly adnexed lamellae (never free), the typical odour like Lactarius quietus, and the constricted spores. The epithet $H$. obrussea is interpreted very variously in the lit. Arnolds (1986) clarified this problem and showed that $H$. quieta is a synonym of the Friesian species Agaricus obrusseus, and he proposed a neotypification. Hygrophorus obrusseus ss. Kuhn. is a different species with free lamellae, without a special odour, with non-constricted spores, and with cheilocystidia. This species was newly described by Arnolds (op.cit.) under the name Hygrocybe cystidiata (Breitenbach \& Kränzlin, 1991).

$$
\text { Hygrophorus agathosmus (Fr.) Fr.: (E), L101, }
$$
mycorrhizal.

Hygrophorus chrysodon (Batsch) Fr.: (E), L97, mycorrhizal.

Hygrophorus eburneus (Bull.) Fr.: (E), L2, mycorrhizal.

Hygrophorus hedrychii (Velen.) K. Kult: (U), L144, mycorrhizal.
Hygrophorus penarius Fr.: (E), L4, L31, L121, mycorrhizal.

Hygrophorus poetarum R. Heim: (E), L13, mycorrhizal.

Hygrophorus pudorinus (Fr.) Fr.: (E), L66, L97, L15, L119, mycorrhizal.

\section{Family Hygrophoropsidaceae}

Hygrophoropsis aurantiaca (Wulfen) Maire: (P), L86, L118, saprobe.

\section{Family Hymenogastraceae}

Galerina badipes (Pers.) Kühner: (P), L66, saprobe.

Gymnopilus sapineus (Fr.) Murrill: (P), L66, saprobe.

Hebeloma leucosarx P.D. Orton: (U), L2, mycorrhizal.

Hebeloma quercetorum Quadr.: (I), (New record for Turkey) 


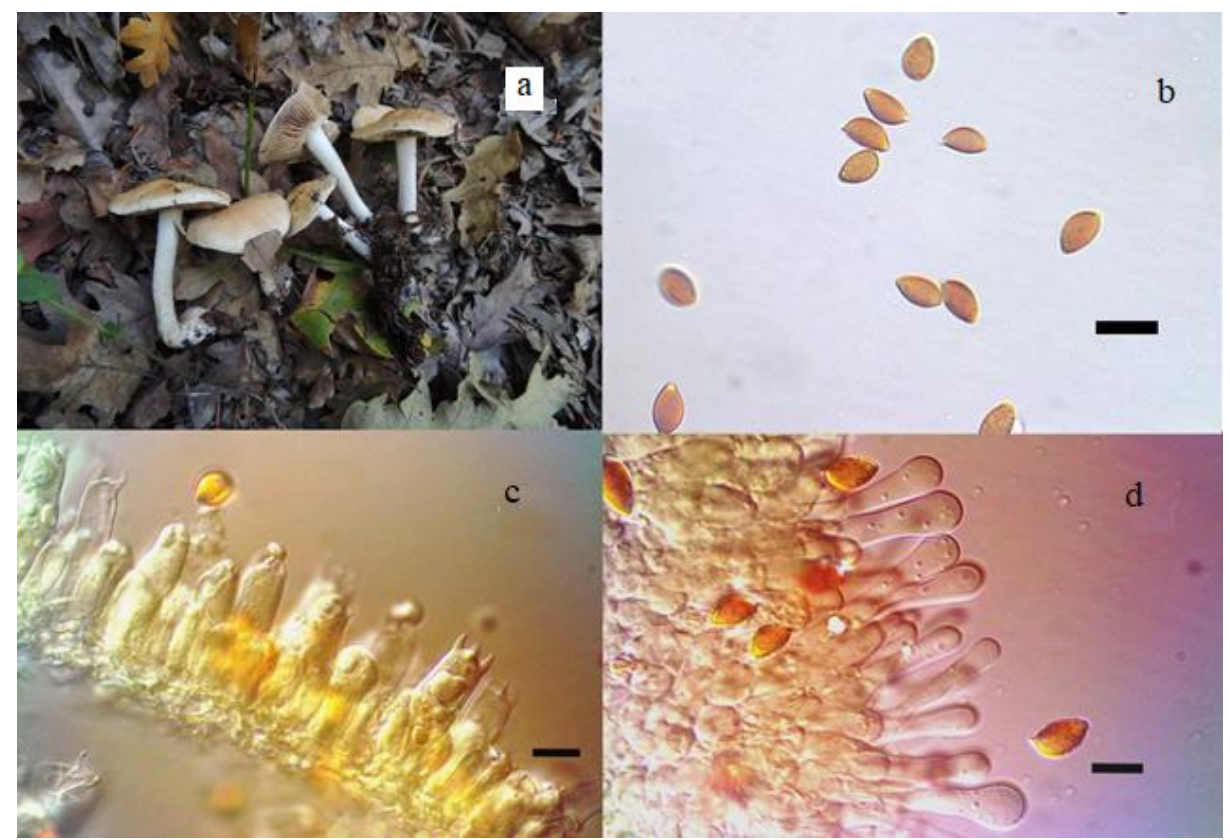

Fig. 4. Hebeloma quercetorum. a) Macroscopic view, b) basidiospores, c) basidia, d) cheilocystidia. Scales $15 \mu \mathrm{m}$.

Pileus 20-50 mm, convex at first, expanded with age, margin decurved for a long time, viscid to slimy, somewhat hygrophanous or not, dark pinkish buff to claybuff or yellowish-brown (Fig. 4a). Flesh elastic and firm, hollow with a hanging string in the stem, white or whitish, with a greyish brown zone over the lamellae. Lamellae deeply emarginate, medium broad to rather broad, fairly crowded, at first pale pinkish buff, then through dark pinkish buff to clay-buff, without droplets. Smell and taste radish-like. Stipe $6-13 \times 28-80 \mathrm{~mm}$, cylindrical or with the base widened to $2 \mathrm{~mm}$, whitish, discolouring to brown from the base, finely pruinose, especially in the upper part. Cortina absent, universal veil not observed. Spore deposit umber. Spores 6-8.5 $\times 10-14 \mu \mathrm{m}$, amygdaloid to broadly citriform, ornamentation distinct to rather strong, dextrinoid (Fig. 4b). Basidia 8-12 × 25-32 $\mu$ m, cylindrical to subclavate, with 4-sterigmata (Fig. 4c). Cheilocystidia $6-8 \times 30-55 \mu \mathrm{m}$, ventricose with a swollen basal part, less often cylindrical or subclavate (Fig. $4 d$ ).

Distribution: L11, under Quercus sp., mycorrhizal.

Remarks: $H$. quercetorum has a mixture of differently shaped cheilocystidia. It has ventricose or lageniform cheilocystidia that are mixed with cylindrical below. There are also usually a few intermediates that are clavatelageniform, i.e. swollen both at the apex and in the basal part. Within Hebeloma. sect. Sinapizantia, and with a large number of ventricose cheilocystidia, can be confused with $H$. sinapizans. However, it is easily separated from $H$. sinapizans macroscopically by the occasional presence of tears, the lower number of lamellae and the less robust appearance, and microscopically by the presence of occasional gently clavate and clavate-lageniform cheilocystidia.
Hebeloma sinapizans (Paulet) Gillet: (P), L90, L120, L121, mycorrhizal.

\section{Family Inocybaceae}

Inocybe acuta Boud.: (P), L36, mycorrhizal.

Inocybe asterospora Quél.: (P), L9, mycorrhizal.

Inocybe catalaunica Singer: (P), L91, L92, L111, mycorrhizal.

Inocybe fuscidula Velen.: (P), L66, L92, mycorrhizal.

Inocybe godeyi Gillet: (P), L73, mycorrhizal.

Inocybe grammopodia Malençon: (P), L73, mycorrhizal.

Inocybe lacera (Fr.) P. Kumm.: (P), L87, mycorrhizal.

Inocybe phaeodisca Kühner var. geophylloides: (P), L66, mycorrhizal.

Inocybe posterula (Britzelm.) Sacc.: (P), L66, L78, L87, L92, L111, mycorrhizal.

Inocybe pseudodestricta Stangl \& J. Veselský: (P), L1, L91, mycorrhizal.

Inocybe queletii Konrad: (P), L127, mycorrhizal.

Inocybe sambucina (Fr.) Quél.: (P), L1, mycorrhizal.

Inocybe splendens R. Heim: (P), L74, L104, mycorrhizal.

Inosperma bongardii (Weinm.) Matheny \& EsteveRav.: (P), L1, mycorrhizal.

Inosperma bongardii (Weinm.) Matheny \& EsteveRav.: (P), L73, L74, mycorrhizal.

Inosperma erubescens (A. Blytt) Matheny \& EsteveRav.: (P), L73, mycorrhizal. 
Family Lycoperdaceae

Bovista plumbea Pers.: (E), L52, L59, L66, L72, L109, L127, saprobe.

Calvatia gigantea (Batsch) Lloyd: (E), L52, L87, saprobe.

Calvatia utriformis (Bull.) Jaap: (E), L66, L72, L95, L118, saprobe.

Lycoperdon atropurpureum Vittad.: (E), L147, saprobe.

Lycoperdon caudatum J. Schröt.: (E), L66, saprobe.

Lycoperdon echinatum Pers.: (E), L19, L31, L50, L53, L66, saprobe.

Lycoperdon excipuliforme (Scop.) Pers.: (E), L96, saprobe.

Lycoperdon lividum Pers.: (E), L82, L87, L135, saprobe.

Lycoperdon mammiforme Pers.: (E), L50, saprobe.

Lycoperdon molle Pers.: (E), L31, L53, L66, L70, L71, L104, L108, L112, L127, saprobe.

Lycoperdon nigrescens Wahlenb.: (E), L74, saprobe.

Lycoperdon perlatum Pers.: (E), L6, L11, L19, L31, L46, L49, L53, L58, L65, L66, L71, L74, L82, L86, L87, L97, L98, L103, L107, L108, L109, L115, L120, L121, L124, L125, L126, L127, saprobe.

Lycoperdon pratense Pers.: (E), L52, L109, saprobe.

Lycoperdon pyriforme Schaeff.: (E), L35, L59, L66, L67, L87, L108, L127, L129, saprobe.

Lycoperdon umbrinum Pers.: (E), L53, saprobe.

Family Lyophyllaceae

Lyophyllum fumosum (Pers.) P.D. Orton: (E), L98, saprobe.

Lyophyllum transforme (Sacc.) Singer: (E), L104, saprobe.

\section{Family Marasmiaceae}

Marasmius bulliardii Quél.: (I), L126, saprobe.

Marasmius cohaerens (Pers.) Cooke \& Quél.: (I), L46, saprobe.

Marasmius oreades (Bolton) Fr.: (E), L66, saprobe.

Marasmius torquescens Quél.: (I), L79, L118, saprobe.

Megacollybia platyphylla (Pers.) Kotl. \& Pouzar: (I), L12, L18, L21, L27, L34, L36, L44, L45, L51, L59, L65, L66, L82, L84, L104, L108, L125, saprobe.

\section{Family Mycenaceae}

Mycena crocata (Schrad.) P. Kumm.: (I), L42, L46, L53, L55, L59, L64, L66, L71, L87, L99, L118, L123, L127, L128, saprobe.

Mycena galericulata (Scop.) Gray: (I), L1, saprobe.
Mycena galopus (Pers.) P. Kumm.: (I), L128, saprobe.

Mycena haematopus (Pers.) P. Kumm.: (I), L7, L79, saprobe.

Mycena laevigata (Lasch) Gillet: (I), L127, saprobe.

Mycena latifolia (Peck) A.H. Sm.: (I), L125, saprobe.

Mycena leptocephala (Pers.) Gillet: (I), L60, saprobe.

Mycena pelianthina (Fr.) Quél.: (P), L128, saprobe.

Mycena pura (Pers.) P. Kumm.: (P), L16, L19, L22, L31, L42, L50, L59, L66, L67, L70, L72, L76, L82, L86, L87, L91, L108, L109, L111, L127, saprobe.

Mycena renati Quél.: (I), L60, saprobe.

Mycena rosea Gramberg: (P), L11, L67, L77, L87, L108, L115, L118, L127, L140, saprobe.

Mycena stipata Maas Geest. \& Schwöbel: (I), L67, saprobe.

Panellus mitis (Pers.) Singer: (I), L115, saprobe.

\section{Family Omphalotaceae}

Collybiopsis confluens (Pers.) R.H. Petersen: (I), L71, saprobe.

Gymnopus dryophilus (Bull.) Murrill: (E), L11, L14, L33, L88, L91, L109, L111, L127, L141, saprobe.

Gymnopus foetidus (Sowerby) P.M. Kirk: (I), L40, saprobe.

Gymnopus fusipes (Bull.) Gray: (I), L84, saprobe.

Gymnopus oreadoides (Pass.) Antonín \& Noordel.: (I), L84, saprobe.

Mycetinis alliaceus (Jacq.) Earle: (I), L1, L16, L17, L42, L57, L59, L60, L64, L65, L66, L71, L74, L76, L79, L87, L91, L92, L97, L108, L109, L113, L114, L115, L127, L128, saprobe.

Omphalotus olearius (DC.) Singer: (P), L11, L15, saprobe.

Rhodocollybia butyracea (Bull.) Lennox: (E), L4, L66, L71, L96, L120, L147, saprobe.

\section{Family Physalacriaceae}

Armillaria cepistipes Velen.: (E), L39, parasite, causes rotten root.

Armillaria gallica Marxm. \& Romagn.: (E), L55, saprobe or weak pathogen.

Armillaria mellea (Vahl) P. Kumm.: (F), L6, L19, L24, L30, L42, L48, L57, L66, L106, L108, L115, L127, L140, parasite, causes rotten root.

Armillaria solidipes Peck: (U), L22, L66, L145, parasite, causes rotten root.

Hymenopellis radicata (Relhan) R.H. Petersen: (E), L6, L11, L16, L18, L21, L22, L30, L33, L34, L35, L36, L37, L38, L41, L42, L44, L46, L47, L50, L53, L64, L65, L66, L67, L70, L71, L72, L74, L78, L82, L84, L85, L91, L92, L104, L108, L109, L118, L119, L123, L125, L126, 
L127, L128, L137, L138, L140, L141, L144, L146, L148, saprobe.

Mucidula mucida (Schrad.) Pat.: (E), L66, L87, L103, L108, L115, L128, L144, saprobe.

Oudemansiella melanotricha (Dörfelt) M.M. Moser: (E), L66, L109, L115, saprobe.

\section{Family Pleurotaceae}

Pleurotus eryngii (DC.) Quél.var. eryngii: (E), L65, mycorrhizal.

Pleurotus ostreatus (Jacq.) P. Kumm.: (F), L87, L90, L147, lignicolous.

Pleurotus pulmonarius (Fr.) Quél.: (E), L32, lignicolous.

\section{Family Pluteaceae}

Pluteus cervinus (Schaeff.) P. Kumm.: (E), L36, saprobe.

Pluteus petasatus (Fr.) Gillet: (I), L27, L31, L36, L50, L140, L144, saprobe.

Pluteus salicinus (Pers.) P. Kumm.: (I), L46, L82, L127, L138, L141, saprobe.

Volvariella bombycina (Schaeff.) Singer: (E), L137, saprobe.

\section{Family Psathyrellaceae}

Britzelmayria multipedata (Peck) D. Wächt. \& A. Melzer: (I), L70, saprobe.

Coprinellus micaceus (Bull.) Vilgalys, Hopple \& Jacq. Johnson: (I), L1, L34, L37, L45, L46, L47, L65, L66, L71, L72, L74, L84, L87, L102, L104, L108, L109, L112, L125, L128, saprobe.

Coprinellus silvaticus (Peck) Gminder: (I), L127, saprobe.

Coprinellus xanthothrix (Romagn.) Vilgalys, Hopple \& Jacq. Johnson: (I), L16, L128, saprobe.

Coprinopsis atramentaria (Bull.) Redhead, Vilgalys \& Moncalvo: (E, or P), L59, L109, saprobe.

Coprinopsis cinerea (Schaeff.) Redhead, Vilgalys \& Moncalvo: (I), L73, saprobe.

Coprinopsis insignis (Peck) Redhead, Vilgalys \& Moncalvo: (I), L9, L11, saprobe.

Coprinopsis lagopus (Fr.) Redhead, Vilgalys \& Moncalvo: (I), L13, saprobe.

Coprinopsis picacea (Bull.) Redhead, Vilgalys \& Moncalvo: (I), L6, L22, L24, L31, L55, L98, L108, L140, saprobe.

Lacrymaria lacrymabunda (Bull.) Pat.: (I), L53, L71, L103, L109, L134, saprobe.

Panaeolus acuminatus (Schaeff.) Quél.: (I), L16, saprobe.
Panaeolus cinctulus (Bolton) Sacc.: (P), : (I), L118, saprobe.

Psathyrella candolleana (Fr.) Maire: (I), L53, saprobe.

Psathyrella cotonea (Quél.) Konrad \& Maubl.: (I), L28, saprobe.

Psathyrella murcida (Fr.) Kits van Wav.: (I), L67, saprobe.

Psathyrella phegophila Romagn.: (I), L148, saprobe.

Psathyrella piluliformis (Bull.) P.D. Orton: (I), L31, saprobe.

Psathyrella tephrophylla (Romagn.) Bon: (I), L13, saprobe.

\section{Family Schizophyllaceae}

Schizophyllum commune Fr.: (M), L34, L60, L69, L82, L87, L95, L112, L113, L138, lignicolous.

Family Strophariaceae

Agrocybe dura (Bolton) Singer: (E), L91, L104, saprobe.

Agrocybe paludosa (J.E. Lange) Kühner \& Romagn. ex Bon: (I), L104, L109, L134, saprobe.

Agrocybe pediades (Fr.) Fayod: (I), L108, saprobe.

Agrocybe praecox (Pers.) Fayod: (E), L1, L21, L59, L60, L82, saprobe.

Hypholoma capnoides (Fr.) P. Kumm.: (I), L2, saprobe.

Hypholoma fasciculare (Huds.) P. Kumm.: (P), L1, L5, L16, L18, L19, L34, L44, L45, L53, L59, L60, L62, L66, L67, L70, L95, L96, L104, L108, L112, L127, L142, L144, saprobe.

Hypholoma lateritium (Schaeff.) P. Kumm: (P), L67, saprobe.

Leratiomyces squamosus (Pers.) Bridge \& Spooner: (I), L53, L66, L87, L118, saprobe.

Pholiota astragalina (Fr.) Singer: (I), L64, saprobe.

Pholiota conissans (Fr.) Kuyper \& Tjall.-Beuk.: (I), L77, saprobe.

Pholiota gummosa (Lasch) Singer: (I), L67, saprobe.

Pholiota lenta (Pers.) Singer: (I), L66, saprobe.

Pholiota mixta (Fr.) Kuyper \& Tjall.-Beuk.: (I), L112, saprobe.

Protostropharia semiglobata (Batsch) Redhead, Moncalvo \& Vilgalys: (E), L109, L118, saprobe.

Stropharia aeruginosa (Curtis) Quél.: (I), L16, L19, L30, saprobe.

Stropharia caerulea Kreisel: (I), L47, L62, L66, L67, L82, L104, L108, L115, L144, saprobe. 


\section{Family Tricholomataceae}

Aspropaxillus candidus (Bres.) M.M. Moser: (E), L2, L66, saprobe.

Atractosporocybe inornata (Sowerby) P. Alvarado, G. Moreno \& Vizzini: (I), L66, saprobe.

Clitocybe costata Kühner \& Romagn.: (I), L45, L73, saprobe.

Clitocybe nebularis (Batsch) P. Kumm.: (F), L47, L61, L64, L66, L96, L97, L104, saprobe.

Clitocybe odora (Bull.) P. Kumm: (E), L50, L65, L66, L96, L109, saprobe.

Clitocybe phaeophthalma (Pers.) Kuyper: (P), L66, saprobe.

Clitocybe phyllophila (Pers.) P. Kumm.: (P), L118, saprobe.

Infundibulicybe geotropa (Bull.) Harmaja: (F), L86, L87, saprobe.

Infundibulicybe gibba (Pers.) Harmaja: (E), L66, L82, L84, L118, saprobe.

Lepista densifolia (J. Favre) Singer \& Clémençon: (E), L73, saprobe.

Lepista nuda (Bull.) Cooke: (E), L19, L66, L67, L78, L123, saprobe.

Melanoleuca exscissa (Fr.) Singer: (E), L145, saprobe.

Paralepista flaccida (Sowerby) Vizzini: (E), L25, L45, saprobe.

Tricholoma acerbum (Bull.) Quél.: (P), L26, mycorrhizal.

Tricholoma albobrunneum (Pers.) P. Kumm.: (P), L19, L62, mycorrhizal.

Tricholoma atrosquamosum var. squarrulosum (Bres.) Mort. Chr. \& Noordel.: (E), L108, mycorrhizal.

Tricholoma aurantium (Schaeff.) Ricken: (P), L66, L96, L119, mycorrhizal.

Tricholoma basirubens (Bon) A. Riva \& Bon: (U), L121, mycorrhizal.

Tricholoma cf. venenatum G.F. Atk.: (U), L77, L96, mycorrhizal.

Tricholoma cingulatum (Almfelt ex Fr.) Jacobashch: (U), L122, mycorrhizal.

Tricholoma equestre (L.) P. Kumm.: (E or P), L108, mycorrhizal.

Tricholoma focale (Fr.) Ricken: (I), L124, mycorrhizal.

Tricholoma fulvum (DC.) Bigeard \& H. Guill.: (E or ?), L57, L108, L125, mycorrhizal.

Tricholoma imbricatum (Fr.) P. Kumm.: (I), L65, L66, mycorrhizal.
Tricholoma joachimii Bon \& A. Riva: (P), L89, mycorrhizal.

Tricholoma populinum J.E. Lange: (E), L20, L61, mycorrhizal.

Tricholoma portentosum (Fr.) Quél.: (E), L39, L62, L121, L124, mycorrhizal.

Tricholoma quercetorum Contu: (U), L40, mycorrhizal.

Tricholoma roseoacerbum A. Riva: (U), (New record for Turkey)

Pileus 50-120 mm, convex with an involute, often ribbed margin, somewhat expanding with age, but margin remaining deflexed or even involute for a very long time, smooth or minutely granulate, slightly viscid in moist weather, almost without radial structure, in the central part pinkish buff to brick or pale vinaceous, somewhat marbled, towards margin whitish to salmon, sometimes with pale yellowish flushes (Fig. 5a). Flesh firm, white to cream; smell weak; taste farinaceous to slightly bitterish. Lamellae emarginate, crowded to very crowded, whitish chrome to cream or straw yellow, often with brown spots when old or damaged. Stipe $15-30 \times 20-40(-60) \mathrm{mm}$, cylindrical to slightly clavate, often somewhat rooting with attenuated base, white or whitish, often pinkish to ochre flushed in the lower part, smooth or slightly punctate floccose. Spores 3-5 $\times$ 4.5-7 $\mu \mathrm{m}$, average, predominantly ellipsoid (Fig. 5b). Basidia 5.0-7.5 × 20-30 $\mu \mathrm{m}$, clavate, with 4sterigmata (Fig. 5c).

Distribution: L66, under A. nordmanniana subsp. bornmuelleriana, mycorrhizal, L114, L20, under $F$. orientalis, mycorrhizal.

Remarks: Tricholoma roseoacerbum is closely related to $T$. acerbum, but differs by the faintly viscid, pinkish buff to the brick cap, and by a less distinctly ribbed cap margin. Another possibility of confusion is $T$. stans, but this species tends to have more well-spaced gills, darker brick cap colours, and a soon expanding cap margin.

Tricholoma saponaceum var. saponaceum (Fr.) $\mathrm{P}$. Kumm.: (U), L71, L108, L127, L4, L144, L119, mycorrhizal.

Tricholoma scalpturatum (Fr.) Quél.: (U), L40, mycorrhizal.

Tricholoma sciodes (Pers.) C. Martín: (U), L114, L144, mycorrhizal.

Tricholoma sejunctum (Sowerby) Quél.: (U), L71, L89, mycorrhizal.

Tricholoma stans (Fr.) Sacc.: (U), L96, mycorrhizal.

Tricholoma subannulatum (Peck) Zeller: (I), L117, L121, L135, mycorrhizal.

Tricholoma sulphureum (Bull.) P. Kumm.: (P), L25, mycorrhizal. 
Tricholoma terreum (Schaeff.) P. Kumm.: (E), L19, L65, L66, L67, L86, L97, L115, L117, L121, mycorrhizal.

Tricholoma triste (Scop.) Quél.: (E), L40, mycorrhizal.

Tricholoma ustaloides Romagn.: (P), L89, L125, mycorrhizal.

Tricholomopsis rutilans (Schaeff.) Singer: (P), L66, L71, L86, L103, L125, L127, saprobe.

Family Tubariaceae

Phaeomarasmius erinaceus (Fr.) Scherff. ex Romagn.: (I), L9, L148, saprobe.

Family Typhulaceae

Typhula fistulosa (Holmsk.) Olariaga: (I), L40, saprobe.

Order Auriculariales

Family Auriculariaceae

Auricularia auricula-judae (Bull.) Quél.: (E), L63, saprobe.

\section{Family Exidiaceae}

Exidia truncata Fr.: (E), L9, L54, L93, L63, L84, saprobe.

Pseudohydnum gelatinosum (Scop.) P. Karst.: (I), L66, L71, L109, saprobe.

Order Boletales

Family Boletaceae

Boletus aereus Bull.: (F), L11, L19, mycorrhizal.

Boletus aestivalis (Paulet) Fr.: (F), L103, L127, L139, mycorrhizal.

Boletus edulis Bull.: (F), L11, L18, L23, L27, L34, L35, L36, L37, L48, L66, L96, L102, L125, L127, L134, L140, L141, mycorrhizal.

Boletus pinophilus Pilát \& Dermek: (F), L11, L65, mycorrhizal.

Boletus reticulatus Schaeff.: (F), L5, L18, L27, L34, L37, L108, L127, mycorrhizal.

Butyriboletus fechtneri (Velen.) D. Arora \& J.L. Frank: (E), L27, L35, L109, L127, mycorrhizal.

Butyriboletus pseudoregius (Heinr. Huber) D. Arora \& J.L. Frank: (E), L11, L89, mycorrhizal.

Butyriboletus regius (Krombh.) D. Arora \& J.L. Frank: (E), L27, mycorrhizal.

Butyriboletus subappendiculatus (Dermek, Lazebn. \& J. Veselský) D. Arora \& J.L. Frank: (E), L5, L19, L66, L67, mycorrhizal.

Caloboletus calopus (Pers.) Vizzini: (I), L57, L66, L125, mycorrhizal.

Chalciporus piperatus (Bull.) Bataille: (E), L126, mycorrhizal.
Cyanoboletus pulverulentus (Opat.) Gelardi, Vizzini \& Simonini: (E), L95, mycorrhizal.

Imperator rhodopurpureus (Smotl.) Assyov, Bellanger, Bertéa, Courtec., Koller,

Loizides, G. Marques, J.A. Muñoz, Oppicelli, D. Puddu, F. Rich. \& P.-A. Moreau: (I), L11, mycorrhizal.

Leccinum aurantiacum (Bull.) Gray: (E), L23, mycorrhizal.

Leccinum duriusculum (Schulzer ex Kalchbr.) Singer: (E), L134, L138, mycorrhizal.

Leccinum pseudoscabrum (Kallenb.) Šutara: (E), L138, mycorrhizal.

Leccinum quercinum (Pilát) E.E. Green \& Watling: (E), L18, mycorrhizal.

Neoboletus erythropus (Pers.) C. Hahn: (E), L11, L59, L66, L70, L71, L72, L74, L78, L91, L92, L125, L127, L141, mycorrhizal.

Neoboletus luridiformis (Rostk.) Gelardi, Simonini \& Vizzini: (E), L125, mycorrhizal.

Neoboletus xanthopus (Klofac \& A. Urb.) Klofac \& A. Urb.: (I), L118, mycorrhizal.

Rubroboletus dupainii (Boud.) Kuan Zhao \& Zhu L. Yang: (P), L65, mycorrhizal.

Rubroboletus rhodoxanthus (Krombh.) Kuan Zhao \& Zhu L. Yang: (U), L11, L66, mycorrhizal.

Rubroboletus satanas (Lenz) Kuan Zhao \& Zhu L. Yan: (P), L11, L125, mycorrhizal.

Strobilomyces strobilaceus (Scop.) Berk.: (I), L46, L127, mycorrhizal.

Suillellus queletii (Schulzer) Vizzini, Simonini \& Gelardi: (E), L11, L70, mycorrhizal.

Suillellus rubrosanguineus (Cheype) Blanco-Dios: (U), L125, mycorrhizal.

Xerocomellus chrysenteron (Bull.) Šutara: (E), L9, L11, L19, L22, L34, L36, L64, L65, L66, L67, L68, L70, L86, L87, L103, L104, L118, L127, L128, L133, L140, L145, mycorrhizal.

Xerocomus depilatus (Redeuilh) Manfr. Binder \& Besl: (E), L29, mycorrhizal.

Xerocomus porosporus (Imler ex G. Moreno \& Bon) Contu: (U), L66, L75, mycorrhizal.

Xerocomus rubellus (Krombh.) Quél.: (E), L66, mycorrhizal.

Xerocomus subtomentosus (L.) Quél.: (E), L12, mycorrhizal.

\section{Family Diplocystidiaceae}

Astraeus hygrometricus (Pers.) Morgan: (I), L39, L42, L48, L61, L94, L95, L113, saprobe. 


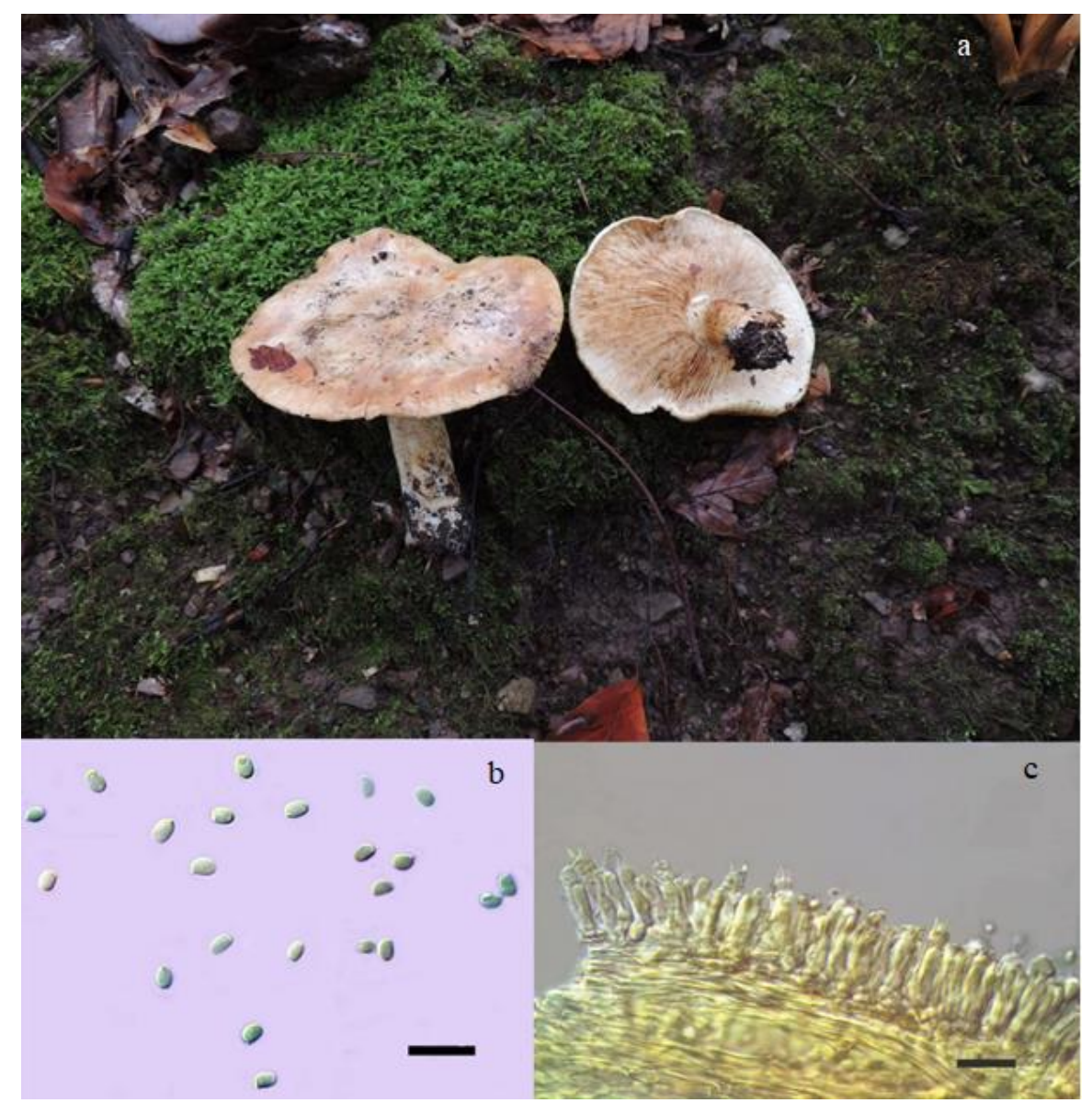

Fig. 5. Tricholoma roseoacerbum. a) Macroscopic view, b) basidiospores, c) basidia. Scales $15 \mu \mathrm{m}$.

\section{Family Gomphidiaceae}

Chroogomphus rutilus (Schaeff.) O.K. Mill.: (E), L19, L31, L38, L49, L58, L62, L66, L82, L86, L94, L103, L107, L109, L121, L127, mycorrhizal.

Family Gyroporaceae

Gyroporus castaneus (Bull.) Quél.: (E), L53, mycorrhizal.

\section{Family Paxillaceae}

Paxillus involutus (Batsch) Fr.: (P), L4, L5, L17, L47, L62, L96, L109, L112, mycorrhizal.

\section{Family Rhizopogonaceae}

Rhizopogon abietis A.H. Sm.: (I), L96, mycorrhizal.

Rhizopogon luteolus Kromb.: (E), L17, L107, L140, mycorrhizal.

Rhizopogon roseolus (Corda) Th. Fr.: (E), L19, L94, L124, mycorrhizal.

\section{Family Sclerodermataceae}

Pisolithus arhizus (Scop.) Rauschert: (M), L145, mycorrhizal.

Scleroderma areolatum Ehrenb.: (I), L95, L144, mycorrhizal.

Scleroderma cepa Pers.: (I), L24, mycorrhizal.
Scleroderma polyrhizum (J.F. Gmel.) Pers.: (I), L144, mycorrhizal.

Scleroderma verrucosum (Bull.) Pers.: (I), L22, L61, mycorrhizal.

\section{Family Suillaceae}

Suillus bovinus (L.) Roussel: (E), L56, L58, mycorrhizal.

Suillus collinitus (Fr.) Kuntze: (E), L15, L117, mycorrhizal.

Suillus granulatus (L.) Roussel: (E), L31, L82, L107, L108, L124, mycorrhizal.

Suillus luteus (L.) Roussel: (E), L17, L19, L24, L66, L96, L104, L124, L127, mycorrhizal.

\section{Family Tapinellaceae}

Tapinella atrotomentosa (Batsch) Šutara: (I), L135, saprobe.

Tapinella panuoides (Fr.) E.-J. Gilbert: (I), L82, saprobe.

\section{Order Cantharellales \\ Family Hydnaceae}

Cantharellus cibarius Fr.: (F), L4, L5, L34, L43, L50, L58, L66, L118, L135, mycorrhizal. 
Clavulina cinerea (Bull.) J. Schröt.: (E), L25, L48, L66, L71, L103, L108, L126, L147, mycorrhizal.

Clavulina coralloides (L.) J. Schröt.: (E), L73, mycorrhizal.

Clavulina cristata (Holmsk.) J. Schröt.: (E), L11, L58, L61, L64, L66, L87, L94, L109, L126, mycorrhizal.

Clavulina rugosa (Bull.) J. Schröt.: (E), L48, L59, L66, L87, L97, L109, L118, L122, L126, L127, mycorrhizal.

Craterellus cornucopioides (L.) Pers.: (F), L4, L42, L47, L48, L50, L59, L66, L71, L89, L104, L108, L109, L114, L118, L126, L144, saprobe.

Craterellus lutescens (Fr.) Fr: (E), L57, L66, L127, mycorrhizal.

Craterellus tubaeformis (Fr.) Quél.: (E), L57, mycorrhizal.

Hydnum repandum L.: (F), L4, L19, L31, L39, L47, L50, L53, L57, L59, L66, L81, L89, L97, L103, L108, L109, L114, L124, L127, L145, mycorrhizal.

Pseudocraterellus undulatus (Pers.) Rauschert: (E), L48, L50, L58, L71, L108, L126, saprobe.

Order Dacrymycetales

Family Dacrymycetaceae

Calocera viscosa (Pers.) Fr.: (I), L66, L71, L87, L123, L127, saprobe, causes a white-rot.

Ditiola radicata (Alb. \& Schwein.) Fr.: (I), L19, L34, L66, L133, saprobe.

Order Geastrales

Family Geastraceae

Geastrum berkeleyi Massee: (I), L115, saprobe.

Geastrum coronatum Pers.: (I), L24, saprobe.

Geastrum fimbriatum Fr.: (I), L58, saprobe.

Geastrum minimum Schwein.: (I), L109, saprobe.

Geastrum triplex Jungh.: (I), L53, under F . orientalis, saprobe. L87, saprobe.

Order Gomphales

Family Clavariadelphaceae

Clavariadelphus pistillaris (L.) Donk: (E), L40, L55, L118, saprobe.

Clavariadelphus truncatus (Quél.) Donk: (E), L14, L53, L70, L71, L109, saprobe.

\section{Family Gomphaceae}

Ramaria aurea (Schaeff.) Quél.: (E), L72, L104, L109, L136, mycorrhizal.

Ramaria flava (Schaeff.) Quél.: (E), L66, mycorrhizal.

Ramaria flavescens Schaeff. ex R.H. Petersen: (E), L31, L66, L70, L127, mycorrhizal.

Ramaria flavobrunnescens (G.F. Atk.) Corner: (E), L34, L109, mycorrhizal.
Ramaria formosa (Pers.) Quél.: (P), L71, , mycorrhizal.

Ramaria lutea Schild: (E), L34, L46, L50, L53, L66, L71, L109, mycorrhizal.

Ramaria pallida (Schaeff.) Ricken: (P), L19, L66, L71, L109, mycorrhizal.

Ramaria rubella (Schaeff.) R.H. Petersen: (U), L63, mycorrhizal.

Ramaria stricta (Pers.) Quél.: (E), L46, L140, mycorrhizal.

\section{Family Lentariaceae}

Lentaria afflata (Lagger) Corner: (I), L104, saprobe.

Order Hymenochaetales

Family Hymenochaetaceae

Coltricia perennis (L.) Murrill: (I), L112, mycorrhizal.

Hymenochaete rubiginosa (Dicks.) Lév.: (I), L112, saprobe.

Inonotus nodulosus (Fr.) P. Karst.: (I), L47, saprobe, causes a soft white-rot.

Inonotus radiatus (Sowerby) P. Karst.: (I), L28, saprobe.

Phellinus hartigii (Allesch. \& Schnabl) Pat.: (I), L109, L115, parasite.

Phellinus lundellii Niemelä: (I), L131, L141, parasite. Family Tubulicrinaceae

Hyphodontia quercina (Pers.) J. Erikss.: (I), L69, L93, saprobe.

Order Hysterangiales

Family Phallogastraceae

Phallogaster saccatus Morgan: (I), L75, saprobe.

Order Phallales

Family Phallaceae

Clathrus ruber P. Micheli ex Pers.: (I), L143, saprobe.

Mutinus caninus (Huds.) Fr.: (I), L45, L139, L140, L142, L146, saprobe.

Phallus impudicus L.: (E), L4, L5, L12, L18, L19, L21, L27, L33, L34, L35, L36, L37, L41, L42, L45, L57, L66, L71, L77, L109, L113, L114, L125, L127, L133, L137, L138, L140, L141, L146, saprobe.

Order Polyporales

Family Fomitopsidaceae

Antrodia ramentacea (Berk. \& Broome) Donk: (I), L129, saprobe.

Daedalea quercina (L.) Pers.: (I), L67, L129, L131, lignicolous.

Fomitopsis pinicola (Sw.) P. Karst.: (M), L1, L5, L67, L70, L71, L74, L75, L97, L104, L108, parasite, causes brown rot. 
Neolentiporus squamosellus (Bernicchia \& Ryvarden) Bernicchia \& Ryvarden: (I), L128, saprobe or weakly parasite, causes a brown rot.

\section{Family Grifolaceae}

Grifola frondosa (Dicks.) Gray: (E), L13, saprobe or also weakly parasite, causes a white-rot and butt rot of trees.

\section{Family Irpicaceae}

Ceriporia reticulata (Hoffm.) Domański: (I), L18, L34, L35, saprobe.

\section{Family Meripilaceae}

Meripilus giganteus (Pers.) P. Karst.: (E), L45, saprobe.

\section{Family Meruliaceae}

Abortiporus biennis (Bull.) Singer: (I), L10, saprobe.

Bjerkandera adusta (Willd.) P. Karst.: (I), L18, L19, L61, L63, L79, L85, L109, L112, L127, saprobe, causes a white-rot.

\section{Family Phanerochaetaceae}

Junghuhnia nitida (Pers.) Ryvarden: (I), L63, L69, saprobe.

Phanerochaete caucasica (Parmasto) Burds: (I), L79, saprobe.

Terana coerulea (Lam.) Kuntze: (I), L61, saprobe.

Family Polyporaceae

Cerrena unicolor (Bull.) Murrill: (I), L148, parasite, causes canker rot.

Cyanosporus subcaesius (A. David) B.K. Cui, L.L. Shen \& Y.C. Dai: (I), L14, lignicolous, causes a brown rot.

Daedaleopsis confragosa (Bolton) J. Schröt.: (I), L6, lignicolous, causes a white-rot.

Faerberia carbonaria (Alb. \& Schwein.) Pouzar: (E), L4, L112, saprobe.

Fomes fomentarius (L.) Fr.: (M), L1, L18, L34, L35, L53, L60, L63, L66, L67, L84, L87, L98, L99, L113, L130, L138, saprobe or parasite, causes a white-rot.

Ganoderma australe (Fr.) Pat.: (I), L115, saprobe or parasite, causes a white-rot.

Ganoderma carnosum Pat.: (I), L14, L53, saprobe or parasite, causes a white-rot.

Ganoderma lucidum (Curtis) P. Karst.: (M), L9, L24, L43, L58, L71, L148, saprobe or parasite, causes a whiterot.

Ganoderma resinaceum Boud.: (I), L108, saprobe or parasite, causes a white-rot.

Lenzites betulinus (L.) Fr.: (I), L64, L112, saprobe.

Neofavolus alveolaris (DC.) Sotome \& T. Hatt.: (M), L36, L148, L51, saprobe.
Picipes badius (Pers.) Zmitr. \& Kovalenko: (E), L1, L16, L18, L21, L34, L37, L45, L59, L66, L68, L76,. L128, L138, L140, saprobe.

Picipes melanopus (Pers.) Zmitr. \& Kovalenko: (I), L12, L27, L60, L67, L108, L139, lignicolous.

Polyporus arcularius (Batsch) Fr.: (E), L4, L11, L47, L57, L84, L102, L129, L133, L138, L141, L148, saprobe.

Polyporus brumalis (Pers.) Fr.: (E), L12, L70, L87, L96, L118, L123, saprobe.

Polyporus ciliatus Fr.: (E), L9, L18, L38, L65, L84, L108, L115, L146, saprobe.

Polyporus meridionalis (A. David) H. Jahn: (E), L36, saprobe.

Polyporus squamosus (Huds.) Fr.: (E), L110, lignicolous.

Polyporus tuberaster (Jacq. ex Pers.) Fr.: (M), L1, L37, saprobe.

Polyporus varius (Pers.) Fr.: (E), L5, L18, L21, L34, L37, L59, L66, L79, L96, L102, L104, L127, L133, L146, saprobe.

Pycnoporus cinnabarinus (Jacq.) P. Karst.: (M), L57, lignicolous.

Trametes gibbosa (Pers.) Fr.: (I), L1, L27, L34, L35, L60, L71, L85, L112, L114, L123, L127, lignicolous.

Trametes hirsuta (Wulfen) Lloyd: (I), L1, L12, L16, L17, L34, L42, L53, L59, L60, L61, L69, L82, L84, L87, L92, L93, L94, L99, L108, L129, L133, L137, lignicolous.

Trametes ochracea (Pers.) Gilb. \& Ryvarden: (I), L12, L16, L21, L67, L87, L108, L138, lignicolous.

Trametes pubescens (Schumach.) Pilát: (I), L6, L108, lignicolous.

Trametes suaveolens (L.) Fr.: (I), L26, lignicolous.

Trametes versicolor (L.) Lloyd: (M), L14, L35, L36, L45, L47, L55, L59, L64, L82, L84, L85, L88, L94, L95, L96, L108, L112, L114, L118, L119, L126, L128, L137, L133, L141, lignicolous.

Trichaptum abietinum (Pers. ex J.F. Gmel.) Ryvarden: (I), L1, L12, L18, L57, L66, L77, L87, L88, L104, L109, saprobe.

Family Sparassidaceae

Sparassis crispa (Wulfen) Fr.: (F), L103, parasite or saprobe on the roots of coniferous trees.

Order Russulales

Family Albatrellaceae

Albatrellus cristatus (Schaeff.) Kotl. \& Pouzar: (I), L16, L48, L55, L66, L71, L94, L118, L122, mycorrhizal.

Albatrellus pes-caprae (Pers.) Pouzar: (I), L59, L125, mycorrhizal. 


\section{Family Amylostereaceae}

Amylostereum areolatum (Chaillet ex Fr.) Boidin: (I), L65, lignicolous, causes a white-rot.

Amylostereum laevigatum (Fr.) Boidin: (I), L96, lignicolous, causes a white-rot.

\section{Family Auriscalpiaceae}

Auriscalpium vulgare Gray: (I), L19, L49, L66, L82, L94, L96, L108, L120, L124, saprobe, on the cones of conifers.

Lentinellus cochleatus (Pers.) P. Karst.: (I), L87, saprobe.

Lentinellus micheneri (Berk. \& M.A. Curtis) Pegler: (I), L4, L34, L45, L111, saprobe.

Lentinellus ursinus (Fr.) Kühner: (I), L146, saprobe.

\section{Family Hericiaceae}

Hericium cirrhatum (Pers.) Nikol.: (E), L18, L21, L27, saprobe or/ and parasite.

Hericium coralloides (Scop.) Pers.: (E), L118, saprobe or/ and parasite.

\section{Family Peniophoraceae}

Peniophora cinerea (Pers.) Cooke: (I), L84, saprobe. Family Russulaceae

Lactarius acerrimus Britzelm.: (I), L125, mycorrhizal.

Lactarius acris (Bolton) Gray: (I), L5, mycorrhizal.

Lactarius blennius (Fr.) Fr.: (I, or P), L118, L139, mycorrhizal.

Lactarius chrysorrheus Fr.: (P), L10, mycorrhizal.

Lactarius deliciosus (L.) Gray: (F), L19, L31, L37, L58, L62, L86, L118, L119, L121, L126, mycorrhizal.

Lactarius evosmus Kühner \& Romagn.: (I), L10, L118, mycorrhizal.

Lactarius ilicis Sarnari: (U), L12, mycorrhizal.

Lactarius illyricus Piltaver: (U), L104, mycorrhizal.

Lactarius lacunarum Romagn. ex Hora: (U), L4, L48, mycorrhizal.

Lactarius mediterraneensis Llistosella \& Bellù: (U), (New record for Turkey)

Pileus 50-100 mm, fleshy, plano-convex at first, soon depressed in the centre, funnel-shaped at the end, gibbous, lobed, margin thin, at first convoluted, then curved. Cuticle thin, elastic, viscous, from dry to shiny, concentrically scrobiculate-guttulata, creamy colour, yellow-fleshed, cream-yellowish, yellow-ocher, with mostly marginal, irregular and scrobicles concentric, darker, ocher-pink or brown-fleshed (Fig. 6a). Flesh medium, thick and firm, then soft, whitish, yellowish and then cream-pink. Faint fruity odour, acrid and bitter taste. Lamellae little spaced gills, from adnate to sub-decurrent, thin, not very elastic, with lamellule, arcuate, sometimes forked and venous-jointed to the stem, cream, creamyellowish, cream-pale ocher, brown-ocher in the injuries. Regular, whole and concolour cutting edge. Milk (Latex) little abundant, fluid, white, yellowish either isolated that on flesh and lamellae. Acre and bitter. Macrochemical reaction: flesh $+\mathrm{KOH}=$ yellow-orange. Stem 15-30 $\times 20$ $40 \mathrm{~mm}$, short and stocky, attenuated cylindrical at the base or truncated cone, even compressed, smooth at the apex, a little guttulated downwards; full, then pithy, fragile and finally hollow, dry, opaque and pruinose, whitish, then cream-whitish, stained with ocher in old age, not scrobiculated. Spores 9-12 × 8-10 $\mu \mathrm{m}$, subglobose, medium size, crested-reticulated, with ridges not very thick, joined by not very thin connections that form mostly complete lattices (Fig. 6f). Basidia 7-10 $\times 45-55 \mu \mathrm{m}$, clavate, with 4sterigmata (Fig. 6b). Macrocheilocystidia 5-7 × 30-50 $\mu \mathrm{m}$, numerous, almost fusiform, attenuated or moniliform at the top. Macropleurocystidia similar to macrocheilocystidia (Fig. 6c).

Distribution: L29, under Quercus sp., mycorrhizal.

Remarks: Similar to L. acerrimus or L. zonarius. It is distinguished by the cap with irregular marginal scrobicles and by the latex which turns yellow both isolated and on flesh and gills.

Lactarius piperatus (L.) Pers.: (E), L9, L148, mycorrhizal.

Lactarius salmonicolor R. Heim \& Leclair: (F), L19, L31, L67, L86, L87, L94, L96, L109, L124, L125, L127, mycorrhizal.

Lactarius semisanguifluus R. Heim \& Leclair: (E), L115, L118, mycorrhizal.

Lactarius turpis (Weinm.) Fr.: (I), L94, mycorrhizal.

Lactarius volemus (Fr.) Fr.: (E), L64, L122, mycorrhizal.

Lactarius zonarius (Bull.) Fr.: (P), L4, L108, mycorrhizal.

Lactifluus bertillonii (Neuhoff ex Z. Schaef.) Verbeken: (I), L71, L146, L148, mycorrhizal.

Lactifluus glaucescens (Crossl.) Verbeken: (P), (New record for Turkey) 


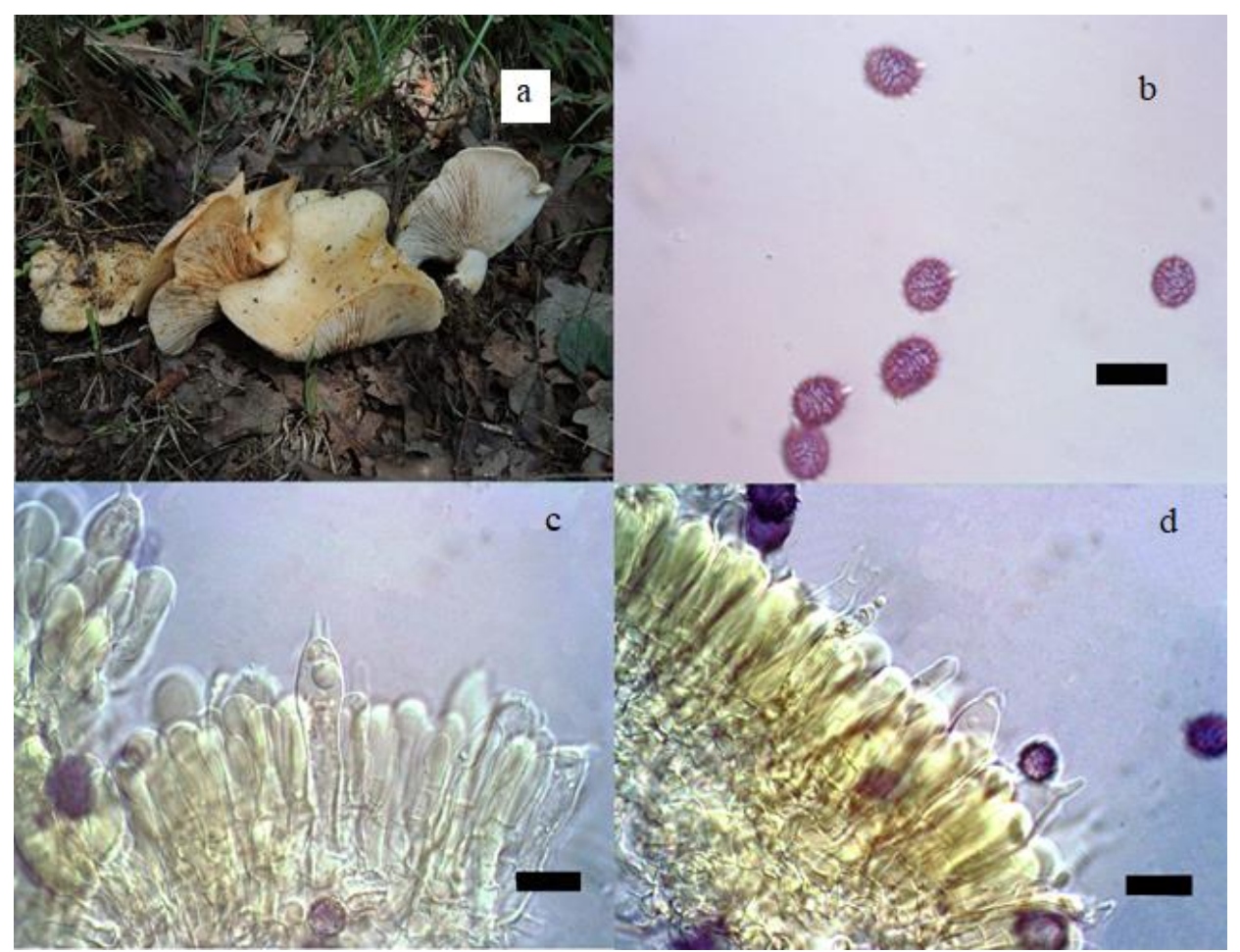

Fig. 6. Lactarius mediterraneensis. a) Macroscopic view, b) basidiospores, c) basidia, d) macropleurocystidia. Scales $15 \mu \mathrm{m}$.

Pileus 40-120 mm, fleshy, flat-convex, soon flat, flatdepressed in the centre, at the end also funnel-shaped, sometimes wavy-lobed, thick margin, long convoluted, then extended, whole, smooth, lobed. The cuticle is thin, adnate, dry, opaque, velvety, often with cracks (in which the greenish colour change of the flesh is evident). Uneven colour hazelnut cream, cream-ocher when ripe, but always lighter at the edge, from white to creamwhitish (Fig. 7a). Flesh thick and firm, compact, then spongy, white, yellowish-cream in the stem, it becomes green, grey-green and finally dark green in the air. Fruity smell, acrid taste. Lamellae thick, thin and low gills, from adnate to sub-decurrent, finally decurrent, arcuate, forked at the stem, with lamellulae, of a cream-whitish colour, then creamy flesh, grey-greenish in the lesions or when rubbed. Whole cutting edge, concolor. Milk (Latex) not abundant, creamy, white at first, then greenish on the flesh and gills, immutable if isolated. Acre. Macrochemical reactions: pileus, stem and latex flesh $+\mathrm{KOH}=$ yelloworange. Stipe $10-25 \times 30-60 \mathrm{~mm}$, robust but not very slender, short, often eccentric or lateral, irregularly cylindrical, sometimes enlarged at the base or compressed, solid and firm, then spongy, dry, opaque, pruinose-velvety, wrinkled, with ocher and finally brown rust in old age or if injured. Spores 5.5-6 × 7-9 $\mu \mathrm{m}$, elliptic, crested, with thin and dense crests, poorly connected, which do not form complete reticles (Fig. 7b). Basidia 7-8.5 $\times 35-45 \mu \mathrm{m}$, clavate, with 4-sterigmata (Fig. 7c). Macrocheilocystidia 5-7 $\times 40-60 \mu \mathrm{m}$, numerous and subcylindrical, obtuse at the apex. Macropleurocystidia alike to macro cheilocystidia, numerous, but larger, 7-10 $\times 60-90 \mu \mathrm{m}$ (Fig. 7d).
Distribution: L9, L143, under Quercus sp., mycorrhizal.

Remarks: Lactifluus glaucescens is closely similar to L. piperatus, which occurs in similar habitats. L. piperatus has the white latex, however, does not turn greenish in the air and does not react with $\mathrm{KOH}$.

Russula acrifolia Romagn.: (I), L4, mycorrhizal.

Russula albonigra (Krombh.) Fr.: (U), L4, mycorrhizal.

Russula alutacea (Pers.) Fr.: (U), L29, mycorrhizal.

Russula amethystina Quél.: (E), L37, mycorrhizal.

Russula amoena Quél.: (E), L96, mycorrhizal.

Russula atropurpurea (Krombh.) Britzelm.: (E), L2, mycorrhizal.

Russula aurea Pers.: (E), L18, L118, mycorrhizal.

Russula aurora Krombh.: (U), L94, mycorrhizal.

Russula brunneoviolacea Crawshay: (E), L73, mycorrhizal.

Russula cavipes Britzelm.: (U), L14, L81, mycorrhizal.

Russula chloroides (Krombh.) Bres.: (E), L11, mycorrhizal.

Russula clariana R. Heim ex Kuyper \& Vuure: (U), L35, L37, L68, L141, mycorrhizal.

Russula cremeoavellanea Singer: (U), L119, mycorrhizal. 
Russula cyanoxantha (Schaeff.) Fr.: (E), L3, L138, mycorrhizal.

Russula delica Fr.: (E), L51, L108, L119, mycorrhizal.

Russula faginea Romagn.: (U), L107, L138, mycorrhizal.

Russula foetens Pers.: (U), L19, mycorrhizal.

Russula fragilis Fr.: (U), L27, L101, mycorrhizal.

Russula gigasperma Romagn.: (U), L19, mycorrhizal.

Russula graveolens Romell: (U), L141, mycorrhizal.

Russula grisea Fr.: (E), L127, L140, mycorrhizal.

Russula insignis Quél.: (U), L12, mycorrhizal.

Russula ionochlora Romagn.: (E), L18, mycorrhizal.

Russula lilacea Quél.: (E, M), (New record for Turkey)

Pileus 30-50 mm across, convex at first, soon flat with a slight central depression, sometimes asymmetrical, obtuse, lobed, the whole then briefly grooved margin, not very fleshy and fragile. Separable cuticle up to and beyond the middle of the radius, dry, pruinose-velvety "opaque" of very variable colour; pink-lilac, reddishpurple, red-vinous, red-carmine, sometimes with brown ocher, cream or pink spots in the centre (Fig. 8a). Lamellae slightly dense, later spaced, free-rounded at the stem, forked to the same, anastomosed with some lamellula, thin, white, dark in old age. Flesh thick but fragile, white, with a slight tendency to grey, odourless and with a sweet taste. Macrochemical reactions flesh + $\mathrm{Fe}=$ brown-red rust, flesh $+\mathrm{F}=$ brown-red, flesh $+\mathrm{SV}=$ carmine red then reddish-brown if dried. Stipe 6-12 $\times 25$ -
$50 \mathrm{~mm}$, initially stiff, soon fragile, cylindrical, sometimes equal at the apex or slightly enlarged towards the base, filled inside, but soon spongy or almost hollow, dry, pruinose then finely wrinkled, white, often tinged with pink or light purple-lilac. Spores 5.5-7 $\times$ 6.5-8.5 $\mu \mathrm{m}$, subglobose-ovoid, warty-echinulate, with both high and sharp and low and obtuse warts, isolated or very rarely joined by some thin tract, amyloid (Fig. 8b). Basidia 10$12 \times 40-50 \mu \mathrm{m}$, with 4-sterigmata (Fig. 8c). Cheilocystidia $8-10 \times 60 \mu \mathrm{m}$, not very numerous, cylindrical-fusiform, pointed at the top. Pleurocystidia similar to Cheilocystidia (Fig. 8d).

Distribution: L112, under C. orientalis, mycorrhizal.

Remarks: Russula brunneoviolacea can occur in the same habitat, and it often has very similar pileal colors and a mild taste. However, it has septate pileocystidia and lacks primordial hyphae. $R$. nitida can also be confused with $R$. lilacea. It likewise has a red-flushed stipe and mild flesh, but it grows with Betula and has an ocher spore deposit. $R$. turci also has a pileus with colours very similar to $R$. lilacea. However, it is associated with conifers such as Picea and Abies and has an ocher-yellow spore deposit.

Russula mairei Singer: (P), L51, mycorrhizal.

Russula nigricans Fr.: (E), L4, L5, L11, L34, L66, mycorrhizal.

Russula odorata Romagn.: (U), L96, mycorrhizal.

Russula olivascens (Fr.) Fr.: (U), L37, mycorrhizal.

Russula pectinatoides Peck: (U), L19, mycorrhizal.

Russula queletii Fr.: (U), L19, mycorrhizal.

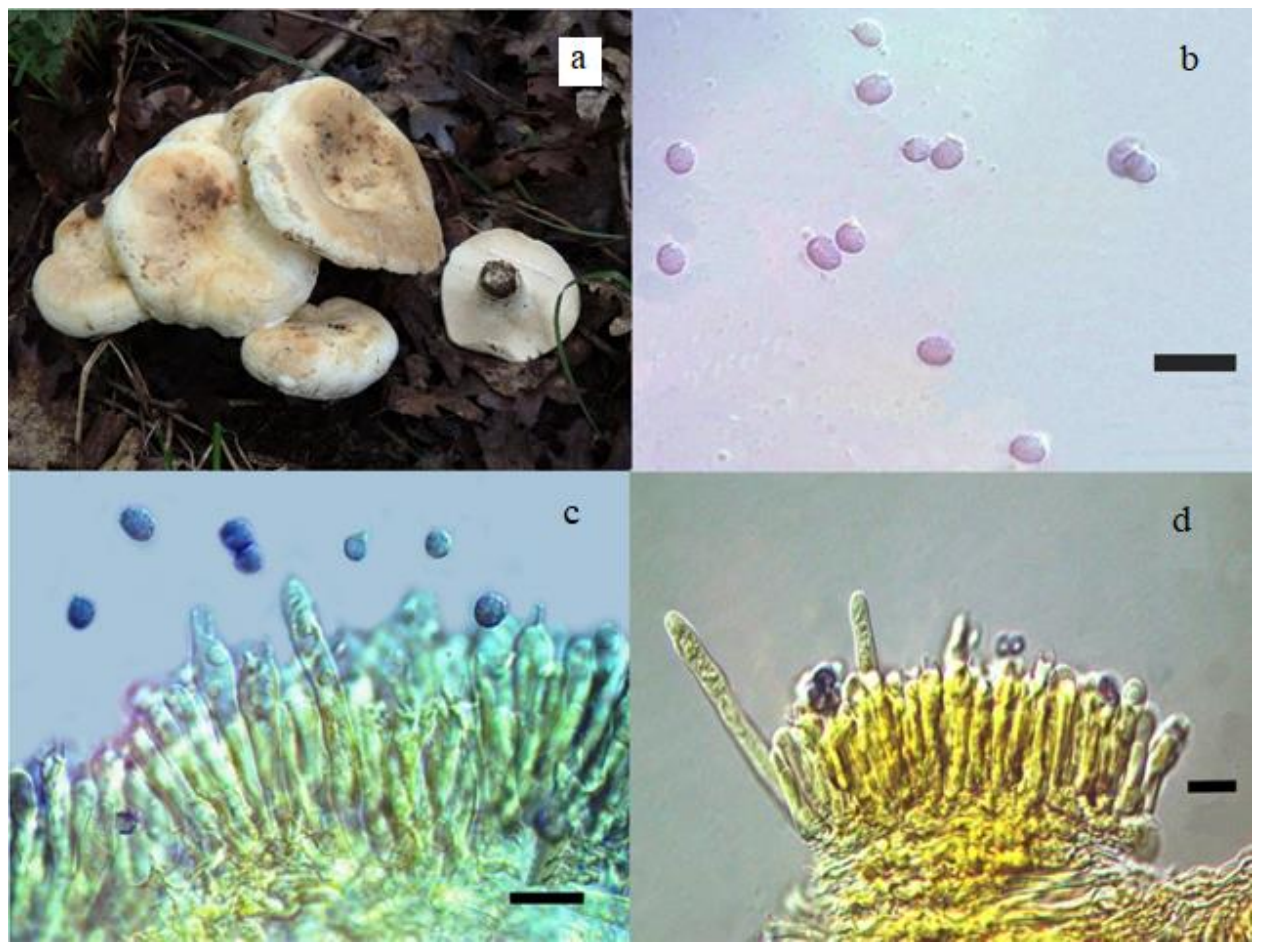

Fig. 7. Lactifluus glaucescens. a) Macroscopic view, b) basidiospores, c) basidia, d) pleurocystidia. Scales $15 \mu \mathrm{m}$. 


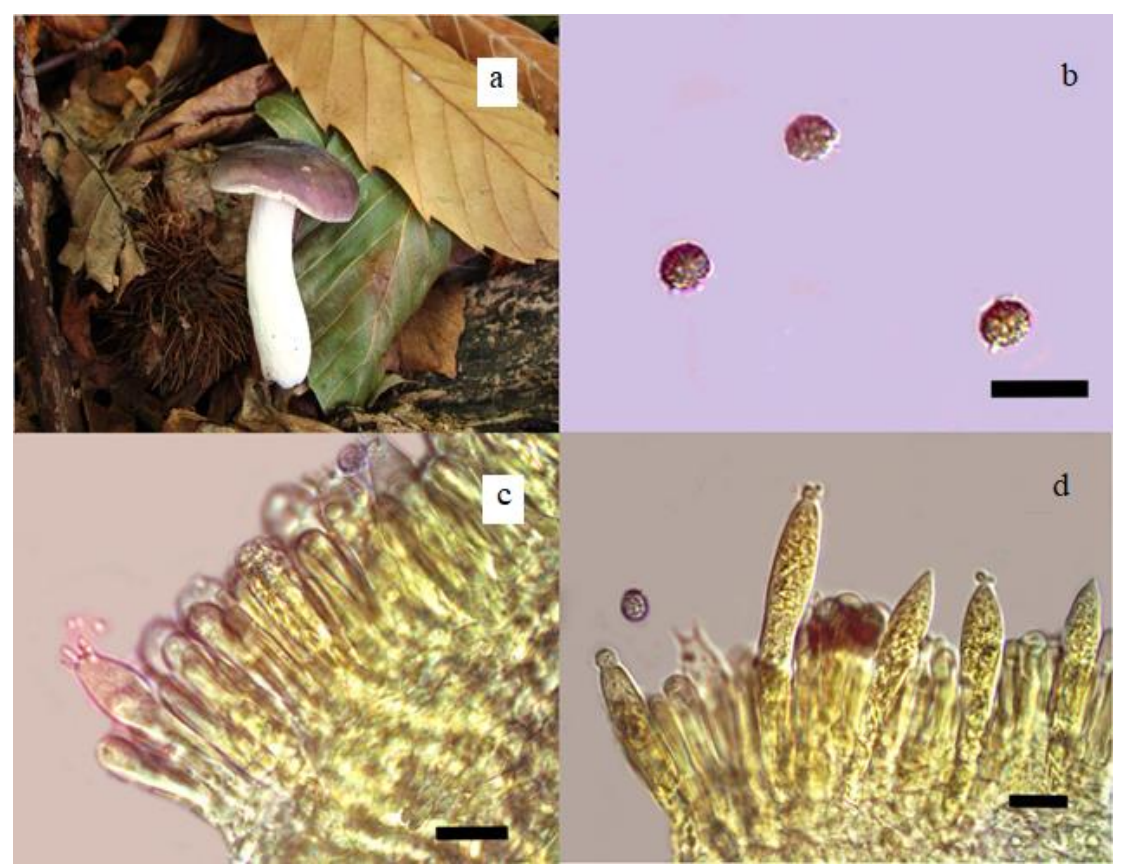

Fig. 8. Russula lilacea. a) Macroscopic view, b) basidiospores, c) basidia, d) pleurocystidia. Scales $15 \mu \mathrm{m}$.

\author{
Russula risigallina (Batsch) Sacc.: (U), L21, \\ mycorrhizal.
}

Russula rubra (Lam.) Fr.: (E), (New record for Turkey).

Pileus 40-100 mm, firm, hemispherical, then convex, finally flat and slightly depressed in the centre, obtuse and regular margin, whole or slightly grooved only when ripe. Cuticle adnate, separable only at the edge, dry, finely pruinose-velvety, of a beautiful dark pink or pink-red colour, red-vermilion or carmine in the centre (Fig. 9a). Lamellae dense then more spaced, subdecurrent then adnexed and free, forked at the stem and anastomosed on the bottom, wide and thick, cream-whitish then light ocher, with whole and concolored cutting edge. Flesh firm and hard, then more tender and soft especially in the stem, white, with a tendency to grey-yellowish, red under the cuticle, with a fruity-honeyed odour and acrid taste also in the gills. Macrochemical reactions flesh $+\mathrm{Fe}=$ yellowish, flesh $+\mathrm{G}=$ deep blue-blue, care $+\mathrm{F}=$ brownish. Stipe $15-$ $30 \times 40-70 \mathrm{~mm}$, firm and robust, cylindrical-clavate or dilated below, attenuated at the base, dry, pruinose then strongly wrinkled especially in old age, full then pithy, white, grey-yellowish at extreme maturity. Spores, 6-8 $\times$ 7-9 $\mu \mathrm{m}$ ovoid, warty-subcrested, with obtuse and hemispherical warts, cone, connected by thin short and irregular or incomplete ridges, amyloid (Fig. 9b). Basidia 9-11 × 30-42 $\mu \mathrm{m}$, clavate to ventricose, with $(1,2) 4$ sterigmata (Fig. 9c). Cheilocystidia 8-12 × 60-100 $\mu \mathrm{m}$, spindle-shaped and slightly bellied, measuring variously appendicular to apex (Fig. 9d). Pleurocystidia are similar to $\mathrm{C}$ species of the genus Cotylidia heilocystidia (Fig. 9e).

Distribution: L132, under P. nigra, mycorrhizal.

Remarks: Russula rosea is very similar to R. rubra. It occurs in comparable habitats, likewise has a finely pruinose pileus, and has hard flesh. However, its flesh is mild, and it has a paler spore deposit, a generally red-flushed stipe, and reticulate spores. The two similar, mild species, $R$. faginea and $R$. pseudointegra also grow in hardwood forests. Russula faginea has a striking herring-like odour and taste, while $R$. pseudointegra has a dark ocher-yellow spore deposit and encrusted primordial hyphae.

Russula sardonia Fr.: (U), L86, mycorrhizal.

Russula sericatula Romagn.: (U), L44, mycorrhizal.

Russula silvestris (Singer) Reumaux: (U), L29, mycorrhizal.

Russula torulosa Bres.: (U), L62, mycorrhizal.

Russula velutipes Velen.: (U), L35, mycorrhizal.

Russula violacea Quél.: (U), L141, mycorrhizal.

Russula violeipes Quél.: (E), L12, L27, mycorrhizal.

Russula virescens (Schaeff.) Fr.: (E), L3, L4, L5, L18, L21, L27, L33, L34, L35, L37, L45, L65, L66, L140, L146, mycorrhizal.

\section{Family Stereaceae}

Aleurodiscus aurantius (Pers.) J. Schröt.: (I), L131, saprobe.

Stereum gausapatum (Fr.) Fr.: (I), L25, lignicolous, causes a white-rot of the heartwood.

Stereum hirsutum (Willd.) Pers.: (I), L4, L12, L14, L57, L62, L63, L66, L69, L80, L82, L84, L85, L93, L108, L112, L113, L127, L133, L136, L137, L139, lignicolous, causes a white-rot of the heartwood.

Stereum insignitum Quél.: (I), L13, causes a white-rot of the heartwood.

Stereum ochraceoflavum (Schwein.) Sacc.: (I), L84, L85, L93, L108, L131, L148, causes a white-rot of the heartwood. 


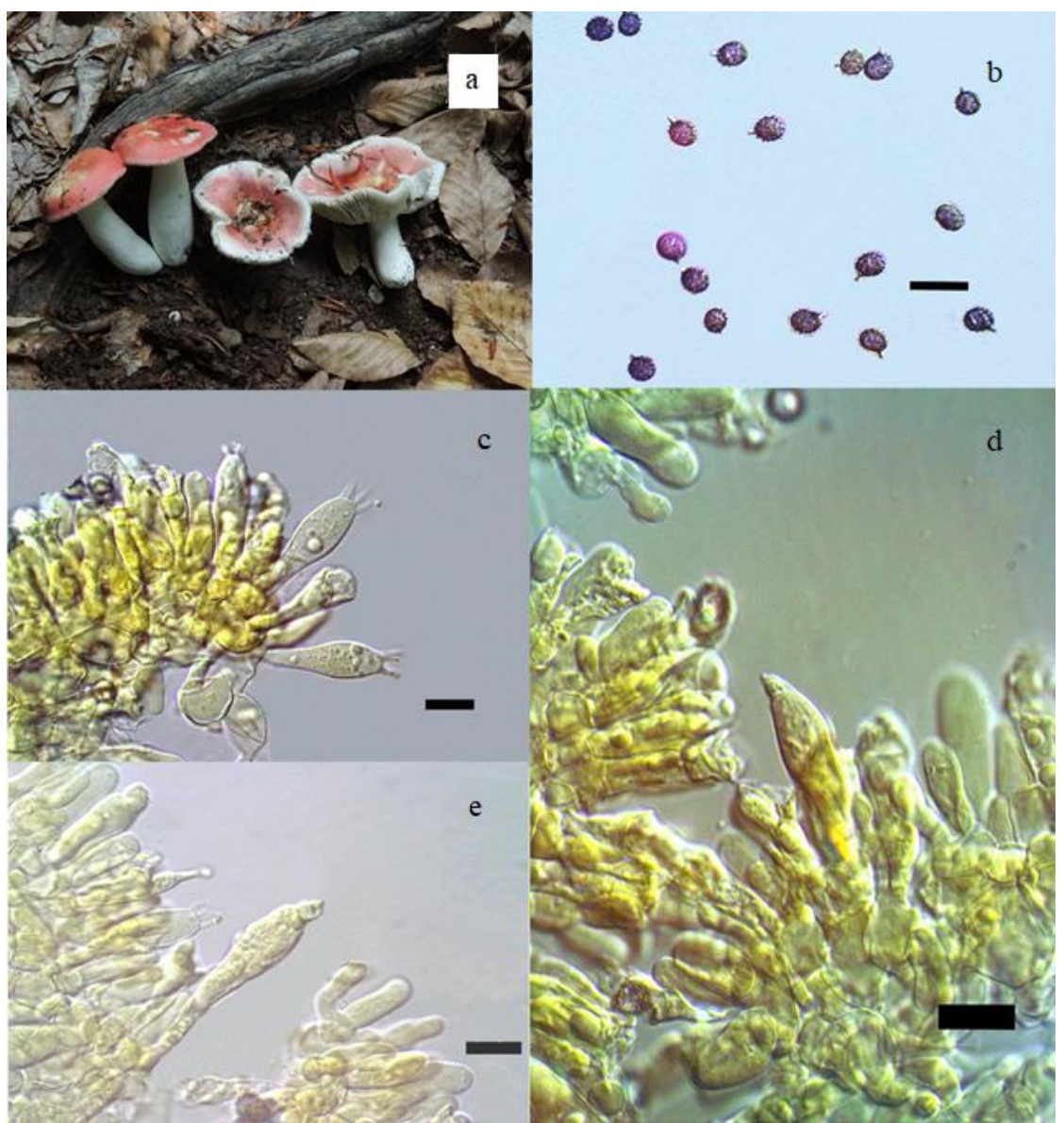

Fig. 9. Russula rubra. a) Macroscopic view, b) basidiospores, c) basidia, d) cheilocystidia, e) pleurocystidia. Scales $15 \mu \mathrm{m}$.

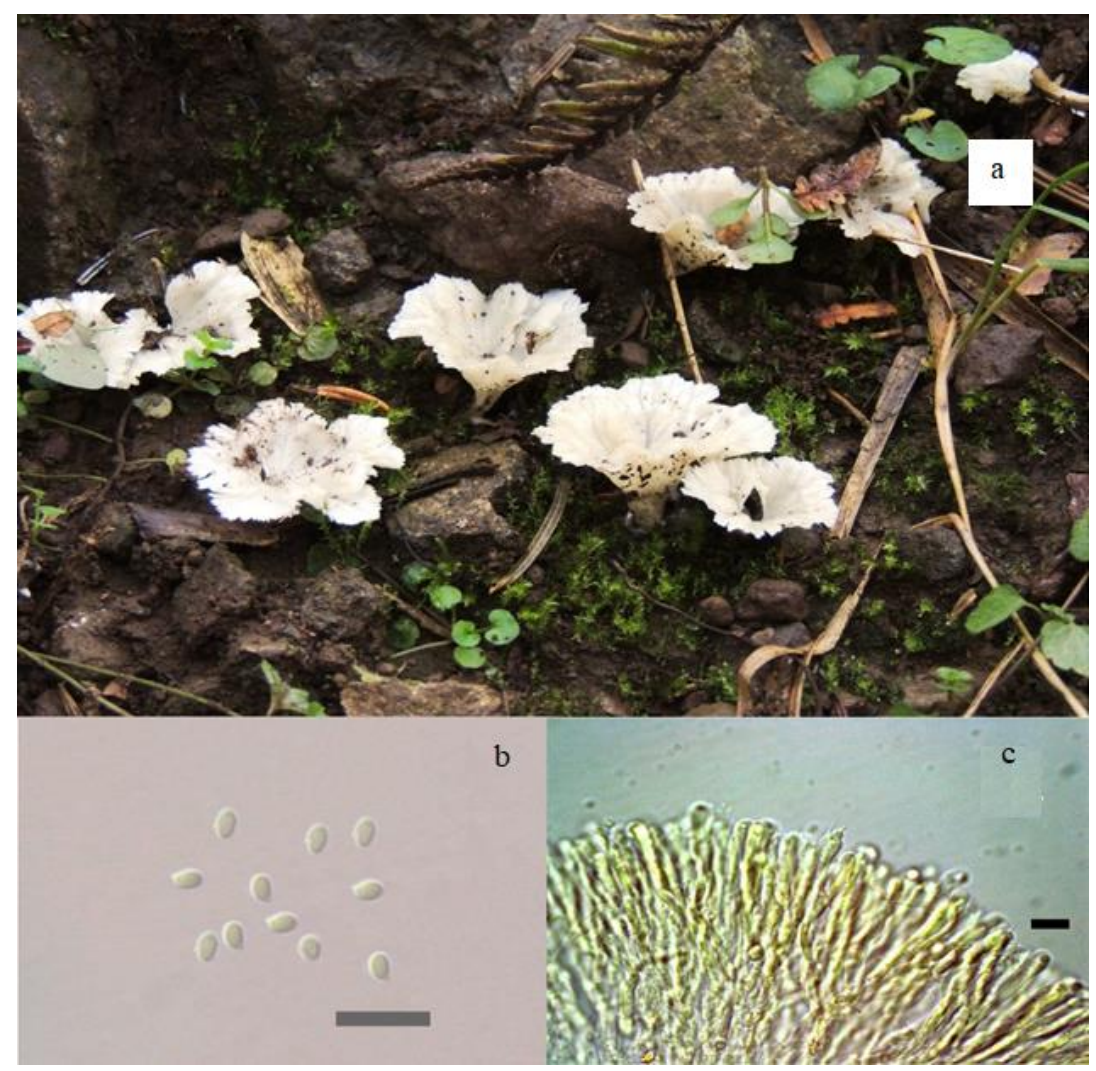

Fig. 10. Stereopsis reidii. a) Macroscopic view, b) basidiospores, c) basidia. Scales $15 \mu \mathrm{m}$. 
Stereum sanguinolentum (Alb. \& Schwein.) Fr.: (I), L56, L126, causes a white-rot of the heartwood.

Stereum subtomentosum Pouzar: (I), L69, L93, L104, causes a white-rot of the heartwood.

\section{Order Stereopsidales \\ Family Stereopsidaceae}

Stereopsis reidii Losi \& A. Gennari: (I), (New Family and Genus record for Turkey)

Basidioma stipitate, stereoid, infundibuliform to spathulate, upper sterile surface whitish to minutely fibrillose, hymenophore smooth to rugose, whitish, margin undulate, finely fimbriate to laciniate (Fig. 10a). Stipe up to $1 \mathrm{~cm}$ long and 1-2 mm in diam, whitish. Basidiospores 3$3.5 \times 4-6 \mu \mathrm{m}$, ellipsoid to ovoid, with a curved and pronounced apiculus, smooth, thin-walled, hyaline (Fig. $10 \mathrm{~b}$ ). Basidia 4-5 × 25-40 $\mu \mathrm{m}$, narrowly clavate, with $4-$ sterigmata, and simple septate at the base (Fig. 10c). Hyphal system 2-6 $\mu \mathrm{m}$ wide, monomitic, hyphae with simple-septa, thin-walled, hyaline. Cystidia not seen.

Distribution: L80, on wood debris of A. nordmanniana subsp. bornmuelleriana, saprobe.

Remarks: Stereopsidaceae family was first described in 2014 to contain the genera Stereopsis by Sjökvist et al. (2014). This genus was classified in the order Polyporales, and Clavulicium genus or in the order Cantharellales until its taxonomical rank has been changed. After detailed Molecular phylogenetics analysis, it has been shown that this genus belongs to in different order. This order might belong in the subclass Phallomycetidae. The Stereopsidales contain corticoid fungi (Clavulicium and Stereopsis) and stalked, funnel-shaped fungi (Stereopsis). The main characteristic of the species is the shape of basidiocarp, at first narrowly ligulate, spathulate to flabelliform, then becoming confluent and forming complicated fructifications, frequently deeply divided into narrow clavarioid or broad lobes. The stipe is short and rudimental. Macroscopically it can be confused with white species of the genus Cotylidia P. Karst, but these have hymenial cystids. A species very similar to the one described is Cyphellostereum pusiolum D.A. Reid, that it has fibulae at the base of the terminal hyphae of the pileic lining and larger spores and polymorphic.

\section{Order Thelephorales \\ Family Bankeraceae}

Hydnellum caeruleum (Hornem.) P. Karst.: (I), L71, L109, mycorrhizal.

Hydnellum concrescens (Pers.) Banker: (I), L2, mycorrhizal.

Hydnellum glaucopus (Maas Geest. \& Nannf.) E. Larss., K.H. Larss. \& Kõljalg: (I), L11, mycorrhizal.

Hydnellum scrobiculatum (Fr.) P. Karst.: (I), L71, mycorrhizal.
Hydnellum suaveolens (Scop.) P. Karst.: (I), L120, mycorrhizal.

Phellodon confluens (Pers.) Pouzar: (I), L96, mycorrhizal.

Phellodon niger (Fr.) P. Karst.: (I), L121, L127, L50, mycorrhizal.

Sarcodon imbricatus (L.) P. Karst.: (E), L19, mycorrhizal.

\author{
Order Tremellales \\ Family Tremellaceae
}

Phaeotremella foliacea (Pers.) Wedin, J.C. Zamora \& Millanes: (I), L34, saprobe.

Tremella mesenterica Retz.: (E), L5, L9, L11, L12, L22, L14, L34, L41, L45, L143, saprobe.

\section{Discussion}

510 macrofungal taxa belonging to 197 genera within 84 families were identified in the research area. Of these, 37 genera and 57 taxa belong to Ascomycota, while 160 genera and 453 taxa belong to Basidiomycota. Nine taxa from Basidiomycota were added to the Turkish Mycobiota as new records. These taxa are Amanita subnudipes, Hebeloma quercetorum, Hygrocybe obrussea, Lactarius mediterraneensis, Lactifluus glaucescens, Russula lilacea, Russula rubra, Stereopsis reidii and Tricholoma roseoacerbum. As mentioned before, there are different kinds of forest ecosystems in the study area which form mixed or pure forests. These areas are both optimal habitats for macrofungi and provide them with a variety of substrates for their growth. Among these habitats, $F$. orientalis and A. nordmanniana subsp. bornmuelleriana forests are very suitable for the growth of macrofungi. The distribution of habitat choices of the macrofungal taxa is as follows: A. nordmanniana subsp. bornmuelleriana 300 species, $F$. orientalis 295 species, Quercus spp. 125 species, $P$. nigra 88 species, $C$. sativa 56 species, $P$. sylvestris 53 species, $C$. orientalis 49 species and $P$. maritima 24 species. Tree species mostly form mixed forests in Samanl1 Mountains. Therefore, dominant species in the mixed forest were taken into account to prepare the distribution of habitat choices. Species with high distribution in A. nordmanniana subsp. bornmuelleriana forests are $H$. radicata (32 different localities (DL), M. alliaceus (29 DL), L. perlatum (27 DL), A. muscaria (24 DL), M. pura (22 DL), C. micaceus (16 DL), and $H$. fasciculare (14 DL). Species with high distribution in $F$. orientalis forests are A. rubescens $(68$ DL), H. radicata (62 DL), P. impudicus (43 DL), $A$. gemmata (27 DL), T. versicolor (22 DL), A. vaginata (21 DL), B. edulis (21 DL), M. procera (20 DL), D. disciformis (19DL), M. platyphila (19 DL), P. varius (17 DL), A. phalloides (15 DL) and $F$. fomentarius (14 DL). Macrofungal diversity which was observed in administrative city borders is as follows: 339 taxa in Sakarya, 265 taxa in Bursa, 227 taxa in Kocaeli and 109 taxa in Yalova. Within these cities, the most and least 
diverse districts were observed as Akyazı (Sakarya) with 217 taxa and Karapürçek (Sakarya) 24 taxa, respectively.

The forests of Akyazı region consist of pure or mixed beech, hornbeam, oak, pine and fir. These forest areas are also in a very healthy condition, providing more suitable place for the growth of macrofungi species. On the other hand, the forests in the Karapürçek region are not healthy and there are many destroyed areas. We can easily see from the available data that mushrooms develop better in parallel with the healthy forest structure.

The numbers of lignicolous and parasitic species are $20(3.7 \%)$ and $18(2.9 \%)$ on different trees, respectively, such as D. quercina on Quercus spp.; S. commune, $P$. squamosus, and $S$. hirsutum on the stump of $A$. nordmanniana subsp. bornmuelleriana; $P$. melanopus, $T$. gibbosa and $T$. ochracea on $F$. orientalis; $C$. subcaesius, T. hirsuta, T. versicolor on C. orientalis; A. cepistipes and $A$. mellea on roots of $F$. orientalis; and $F$. fomentarius on the stump of $A$. nordmanniana subsp. bornmuelleriana, $F$. orientalis, Quercus sp. and C. orientalis; F. pinicola on trunk of $P$. nigra, $P$. sylvestris and $F$. orientalis. Moreover, 245 (48\%) species are saprobe, 226 (45\%) are mycorrhizal, and 1 species is entomopathogenic (Ophiocordyceps gracilis). Overall graphic about ecological statuses of the species is given in Fig. 11.

According to the reviewed literature data (Boa 2004, Hall et al. 2016) 204 (40\%) of the 510 taxa are inedible, 7 $(1.37 \%)$ are edible or suspicious, $12(12.36 \%)$ are used for medical purposes, $153(30 \%)$ are edible, $65(12.75 \%)$ are poisonous, $19(3.73 \%)$ are used as food, $48(9.4 \% 100)$ are with unknown status and $2(0.4 \%)$ are edible or poisonous. Among the edible and used as food taxa, 16 are collected and consumed in the region by Vill.rs. Members of the genus Morchella are known as "Kuzu göbeğì", $M$. procera as "Dedebörü, şemsiye mantarı", $P$. ostreatus as "Kavak mantarı, geyik mantarı", L. deliciosus and L. salmonicolor as "Kanlica", A. caesarea as "Gelincik mantarı, yumurta mantarı, sarı paça", $A$. campestris as "Çayır mantarı, içi kızıl", C. comatus as "Söbelen”, B. edulis as "Ayı Mantarı, sünger mantarı",

\section{Ecological Status}

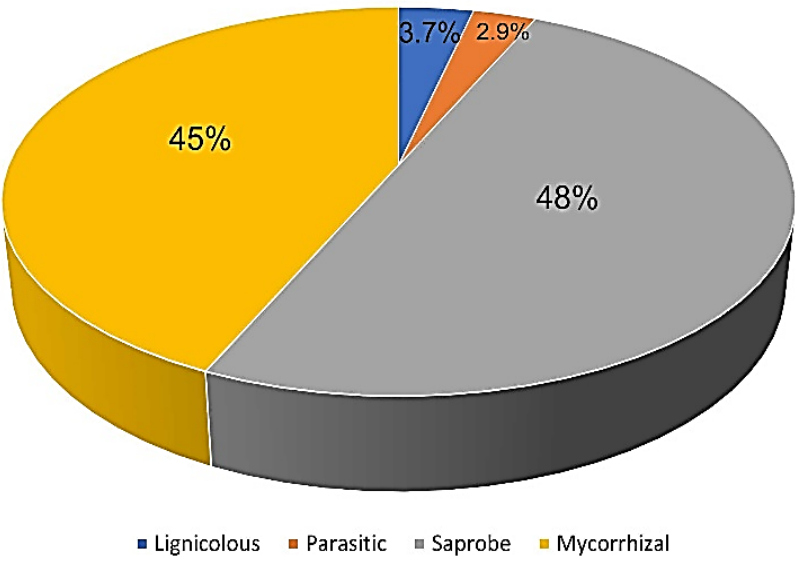

Fig. 11. Ecological status of the species.
Table 2. Similarity percentages of Samanl1 Mountains with neighbouring studies in terms of macrofungal species.

\begin{tabular}{lccc}
\hline \multicolumn{1}{c}{ Study } & $\begin{array}{c}\text { Number of } \\
\text { identical } \\
\text { taxa }\end{array}$ & $\begin{array}{c}\text { Total } \\
\text { taxa }\end{array}$ & $\begin{array}{c}\text { Similarity } \\
\text { percentage } \\
(\%)\end{array}$ \\
\hline \hline Kocaeli (Akata et al. 2018) & 91 & 131 & 17.84 \\
Bursa Gücin et al. 1995) & 56 & 84 & 10.98 \\
İznik (All et al. 2016) & 58 & 91 & 11.37 \\
Yalova (All et al. 2017) & 42 & 78 & 8.23 \\
Samanlı Mountains & & 510 & \\
\hline \hline
\end{tabular}

A. mellea as "Bal mantarı", I. geotropa as "Malkadın", $C$. nebularis as "Cincile", $H$. repandum as "Geyik dili", $S$. crispa as "Kıvırcık", C. cibarius as "Kaz ayağı, Sar1 kulak", C. cornucopioides as "Borazan mantar1, kara borazan". Poisonous species of the area are $E$. rhodopolium, E. sinuatum, A. gemmata, A. muscaria, A. pantherina, A. phalloides, A. solitaria, A. virosa, A. xanthoderma, $P$. cinctulus, $H$. fasciculare, I. bongardii var. bongardii, I. bongardii var. pisciodora, I. erubescens, I. fuscidula, I. lacera, I. leiocephala, I phaeodisca var. geophylloides, I. posterula, I. pseudodestricta, I. queletii, I. sambucina, I. splendens, I. acuta, I. asterospora, C. humicola, C. orellanus, L. acris, L. chrysorrheus, $R$. mairei, C. calopus, B. satanas, $H$. aurantiaca, $P$. involutus, $C$. phaeophtalma, C. phyllophila, $M$. pelianthina, M. pura, M. rosea, T. sulphureum, $H$. lacunosa, and $H$. crispa.

There exist fungal data on some nearby regions of our study area with former studies in İznik (Allı et al. 2016), Yalova (All et al. 2017), Bursa (Gücin et al. 1995) and in Kocaeli (Akata et al. 2018). The comparative distribution of the species numbers identified in these studies is given in Table 2. The results of this work showed a few similarities with the findings of the studies carried out in neighbouring regions. The number of identical taxa and similarity percentages of relevant studies are given in Table 2. According to the table, the number of taxa that are common in each study was found as twelve, and these species are A. pantherina, A. mellea, C. cibarius, $F$. fomentarius, $H$. fasciculare, L. betulinus, $L$. nuda, $L$. pyriforme, $M$. procera, $P$. ostreatus, $S$. commune, $S$. aeruginosa, $T$. versicolor, and $X$. hypoxylon. The similarity rates of the studies are $17.84 \%$ for Kocaeli (Akata et al. 2018), 10.98\% for Bursa region (Gücin et al. 1995), 11.37\% for İznik Region (All et al. 2016), and $8.23 \%$ for Yalova region (All1 et al. 2017).

\section{Acknowledgement}

We appreciate the help of Adapazarı Regional Directorate of Forestry and Zekeriya Beyazlı (Chief of Akyazı Forest Management Department, Turkey), Bursa 
Regional Directorate of Forestry, and Turgut Keskin (Manager of Non-Wood Products and Services, Turkey) for the logistic support in collecting of the specimens.

Ethics Committee Approval: Since the article does not contain any studies with human or animal subject, its approval to the ethics committee was not required.

Author Contributions: Material supplying: H.H.D., Ö.Ö., M.A.Ş., Data acquisition: H.H.D., Ö.Ö.

\section{References}

1. Akata, I., Kabaktepe, Ş., Sevindik, M. \& Akgül, H. 2018. Macrofungi determined in Yuvacık Basin (Kocaeli) and its close environs. Kastamonu University Journal of Forestry Faculty, 18(2): 152-163.

2. All1, H., Candar, S.S. \& Akata, I. 2017. Macrofungal Diversity of Yalova Province. The Journal of Fungus, 8(2): 76-84.

3. Allı, H., Şen, İ. \& Altuntaş, D. 2016. Macrofungi of İznik Province. Communications Faculty of Science University of Ankara Series C Biology Geological Engineering and Geophysical Engineering, 25(1-2): 7-24.

4. Arnolds, E. 1986. Notes on Hygrophoraceae - VI. Persoonia, 13: 57-68.

5. Basso, M.T. 1999. Fungi Europaei. Vol.7. Mykoflora, Alassio, 767 pp.

6. Bernicchia, A. 2005. Fungi Europaei. Vol.10. Edizioni Candusso, Alassio, 808 pp.

7. Boa, E. 2004. Wild Edible Fungi A global overview of their use and importance to people. Food and Agriculture Organization of the United Nations, Rome, $147 \mathrm{pp}$.

8. Breitenbach, J. \& Kränzlin, F. 1984. Fungi of Switzerland. Ascomycetes Vol.1: Ascomycetes. Verlag Mykologia. Lucerne, $310 \mathrm{pp}$.

9. Breitenbach, J. \& Kränzlin, F. 1986. Fungi of Switzerland. Vol.2: Heterobasidiomycetes, Aphyllopharales, Gasteromycetes. Verlag Mykologia. Lucerne, 412 pp.

10. Breitenbach, J. \& Kränzlin, F. 1991. Fungi of Switzerland. Vol.3: 1st Part, Boletes and Agaricus. Verlag Mykologia. Lucerne, $361 \mathrm{pp}$.

11. Breitenbach, J. \& Kränzlin, F. 1995. Fungi of Switzerland, Vol.4: 2nd Part Agaricus. Verlag Mykologia. Lucerne, 368 pp.

12. Breitenbach, J. \& Kränzlin, F. 2000. Fungi of Switzerland, Vol.5: Agarics part 3, Cortinariaceae. Verlag Mykologia. Lucerne, 338 pp.

13. Candusso, M. \& Lanzoni, M. 1990. Fungi Europaei. Vol.4. Libreria Editrica Biella Giovanna, Saronno, 743 pp.

14. Candusso, M. 1997. Fungi Europaei. Vol.6 Libreria Basso, Alassio, 783 pp.

15. Cannon, P.F. \& Kirk, P.M. 2007. Fungal Families of the World. CABI Publishing, Wallingford, $456 \mathrm{pp}$.

16. Christensen, M. \& Heilmann-Clausen, J. 2013. The Genus Tricholoma. Narayana Press, Gylling, 227 pp.
Conflict of Interest: The authors have no conflicts of interest to declare.

Funding: This research is financially supported by The Scientific and Technological Research Council of Turkey (TÜBİTAK, TBAG 112T136) and Selçuk University, Scientific Research Projects Coordinating Office (BAP/13401072).

17. Eriksson, J. \& Ryvarden, L. 1973. The Corticiaceae of North Europe, Vol.2: Aleurodiscus-Confertobasidium. Fungiflora. Oslo, $231 \mathrm{pp}$.

18. Eriksson, J. \& Ryvarden, L. 1976. The Corticiaceae of North Europe, Vol.4: Hyphodermella-Mycoacia. Fungiflora, Oslo, $337 \mathrm{pp}$.

19. Eriksson, J., Hjortstam, K. \& Ryvarden, L. 1978. The Corticiaceae of North Europe, Vol.5: MycoaciellaPhanerochaete. Fungiflora, Oslo, 158 pp.

20. Eriksson, J., Hjortstam, K. \& Ryvarden, L. 1984. The Corticiaceae of North Europe, Vol.7: SchizoporaSuillosporium. Fungiflora, Oslo, 166 pp.

21. Galli, R. 2003a. I Tricholomi. 2a edizione. Edinatura, Milano, $271 \mathrm{pp}$.

22. Galli, R. 2003b. Le Russule. 2a edizione. Roberto Galli, Milano, $480 \mathrm{pp}$.

23. Galli, R. 2004. Gli Agaricus. 1a edizione. Dalla Natura, Milano, $216 \mathrm{pp}$.

24. Galli, R. 2006. I Lattari. 1a edizione. Dalla Natura, Milano, 299 pp.

25. Galli, R. 2007a. I Boleti. 3a edizione. Dalla Natura, Milano, $293 \mathrm{pp}$.

26. Galli, R. 2007b. Le Amanite. 2a edizione. Dalla Natura, Milano, $216 \mathrm{pp}$.

27. Gücin, F., Solak, M.H. \& Işı1loğlu, M. 1995. Mushrooms of Uludağ (Bursa-Turkey), 402-413. Paper presented at the Plant Life in Southwest and Central Asia Symposium, 2128 May, İzmir-Turkey.

28. Hall, I.R., Lyon, T., Yun, W. \& Buchanan, P. 2016. Truffles and Mushrooms, A list of putative edible or medicinal ectomycorrhizal mushrooms. Truffles \& Mushrooms (Consulting) Ltd., Dunedin, 45 pp.

29. Hjortstam, K., Larsson, K.-H. \& Ryvarden, L. 1987. The Corticiaceae of North Europe, Vol.1: Introduction and Keys. Fungiflora, Oslo, 59 pp.

30. Hjortstam, K., Larsson, K.-H. \& Ryvarden, L. 1988. The Corticiaceae of North Europe, Vol.8: Phlebiella, Thanatephorus-Ypsilonidium. Fungiflora, Oslo, 181 pp.

31. Horak, E. 2005. Röhrlinge und Blätterpilze in Europa. Elsevier, Munich, 555 pp.

32. Index Fungorum. www.indexfungorum.org; (Date accessed: 15.09.2021) 
33. Kirk, P.F., Cannon, P.F., Minter, D.W. \& Stalpers, J.A. 2008. Dictionary of the Fungi. CAB International, Wallingford, $445 \mathrm{pp}$.

34. Knudsen, H. \& Vesterholt, J. 2008. Funga Nordica: Agaricoid, Boletoid, Cyphelloid Genera. Nordsvamp, Copenhagen, $965 \mathrm{pp}$.

35. Kränzlin F., 2005. Fungi of Switzerland, Vol.6: Russulaceae, Lactarius, Russula. Verlag Mycologia, Luzern, 317 pp.

36. Medardi, G. 2006. Ascomiceti d'Italia. A.M.B, Venice, 678 pp.

37. Michael, W.B, Alan, E.B. \& Arleen, R.B. 2014. Ascomycete Fungi of North America: a mushroom reference guide. University of Texas Press, Austin, 488 pp.

38. Moser, M. 1983. Keys to Agarics and Boleti. Gustav Fischer Verlag, Stuttgart, 535 pp.

39. Muñoz, J.A. 2005. Fungi Europaei. Vol.2. Edizioni Candusso, Alassio, 952 pp.

40. Mycobank. https://www.mycobank.org; (Date accessed: 15.09.2021)

41. Neville, P. \& Poumarat, S. 2004. Fungi Europaei. Vol.9. Edizioni Candusso, Alassio, $1120 \mathrm{pp}$.

42. Parra, L.A. 2008. Fungi Europaei. Vol.1. Edizioni Candusso, Alassio, 522 pp.
43. Riva, A. 2003a. Fungi Europaei. Vol.3. Edizioni Candusso, Alassio, 826 pp.

44. Riva, A. 2003b. Fungi Europaei. Vol.3A. Edizioni Candusso, Alassio, 200 pp.

45. Robich, G. 2007. Mycena D'Europe. A.M.B, Trento, 728 pp.

46. Ryvarden, L. \& Gilbertson, R.L. 1993. European Polypores. Vol.1. Fungiflora, Oslo, 387 pp.

47. Ryvarden, L. \& Gilbertson, R.L. 1994. European Polypores. Part 2: Meripilus-Tyromyces. Fungiflora, Oslo, p: 394-743.

48. Sesli, E., Asan, A., Selçuk, F. (eds.), Abacı Günyar, Ö., Akata, I., Akgül, H., Aktaş, S., Alkan, S., Allı, H., Aydoğdu, H., Berikten, D., Demirel, K., Demirel, R., Doğan, H.H., Erdoğdu, M., Ergül, C.C., Eroğlu, G., Giray, G., Haliki Uztan, A., Kabaktepe, Ş., Kadaifçiler, D., Kalyoncu, F., Karaltı, İ., Kaşık, G., Kaya, A., Keleş, A., Kırbağ, S., Kıvanç, M., Ocak, İ., Ökten, S., Özkale, E., Öztürk, C., Sevindik, M., Şen, B., Şen, İ., Türkekul, İ., Ulukap1, M., Uzun, Ya., Uzun, Yu. \&Yoltaş, A. 2020. Türkiye Mantarları Listesi. Ali Nihat Gökyiğit Vakfı Yayını, İstanbul, 1177 pp.

49. Sjökvist, E., Pfeil, Bernard E., Larsson, E. \& Larsson, K-H 2014. Stereopsidales - A New Order of MushroomForming Fungi. Plos One, 9(4): e95227.

50. Tullos, R.E. 2000. Nomenclatural changes in Amanita. Mycotaxon, 75: 329-332. 